\title{
Control of Dynamic Stability during Gait Termination on a Slippery Surface
}

by

Alison Robyn Oates

\author{
A thesis \\ presented to the University of Waterloo \\ in fulfillment of the \\ thesis requirement for the degree of \\ Doctor of Philosophy \\ in
}

Kinesiology

Waterloo, Ontario, Canada, 2007

(C) Alison R Oates 2007 


\section{AUTHOR'S DECLARATION FOR ELECTRONIC SUBMISSION OF A THESIS}

I hereby declare that I am the sole author of this thesis. This is a true copy of the thesis, including any required final revisions, as accepted by my examiners.

I understand that my thesis may be made electronically available to the public. 


\begin{abstract}
The purpose of this thesis is to investigate the reaction to a purely unexpected slip during gait termination and subsequent experiences stopping on the slippery surface in participants who are young and healthy, older and healthy and who have Parkinson's disease while on traditional dopamine-replacement medication.

Gait termination requires control of the forward momentum of the body's centre of mass (COM). This forward momentum must be dissipated and the COM held within a newly formed base of support. The challenge of stopping on a slippery surface involves maintaining stability while transitioning from steady-state locomotion to steady-state stance. Experience with a slippery surface changes postural and gait characteristics to diminish the perturbing effect of the slip. The magnitude of the slip response diminishes quickly as the movement becomes more efficient.
\end{abstract}

Our investigations revealed a typical slip response to a purely unexpected slip during gait termination including a lowering of the COM, an increased muscular response to support the body, a shortened step and an arm raise. Knowledge of and experience with the slippery surface quickly changed the slip response to reduce the perturbing effect of the slip and also to increase the efficiency of the response while smoothly transitioning from steady-state locomotion to gait termination.

Parkinson's disease impairs balance control, the ability to switch between motor tasks and also to stop within two steps. The need for a voluntary change in motor programs along with difficulty stopping and increased instability makes gait termination a potentially 
difficult task for someone with Parkinson's disease (PD). The participants with PD used a slower, safer strategy to stop on non-slippery surfaces to compensate for their instability compared to age-matched controls. When a slip was first introduced during gait termination, the participants with PD continued to be less stable in the plane of progression than the control group. Despite the instability, the PD group was still able to integrate a balancecorrecting response into a voluntary gait termination program. The ability to generate adaptive strategies to integrate the balance-correcting response into a voluntary gait termination program over multiple trials does not appear to be affected by PD; both the control group and PD group showed behavioural modifications according to repeated exposures to the slippery surface. Although participants with PD seemed slightly less stable and walked slower, their behavioural adaptations were similar to the control group. 


\section{Acknowledgements}

I would like to acknowledge the support and mentoring of Drs. Aftab Patla and Jim Frank through this process. Their dedication to research excellence and care for their students is inspiring.

I would like to thank my colleagues in the GaP lab that have helped shaped this thesis including Dr. Milad Ishac, Michael Cinelli and Michael Greig. I would like to acknowledge and thank Dr. Fay Horak and her research team at the Balance Disorders Laboratory at the Oregon Health Sciences University for their assistance and patience during the collection of the studies involving people with Parkinson's disease, especially Patty Carlson-Kuhta, Edward King, Triana Nagel-Nelson and Andy Owings.

Erin Harvey provided much appreciated statistical consulting and I would like to thank her for her time and support.

Finally, I need to acknowledge and thank Karen VanOoteghem for her unending support in the form of data collection, processing, brainstorming, problem solving and, most importantly, friendship. 


\section{Dedication}

I would like to dedicate this thesis to my parents for their unending support and for their examples in the pursuit of higher education.

I also want dedicate this thesis to my husband David for the encouragement, support and for always believing in me. Thank you for being my best friend, a great husband, and my biggest fan! 


\section{Table of Contents}

AUTHOR'S DECLARATION FOR ELECTRONIC SUBMISSION OF A THESIS ............. ii

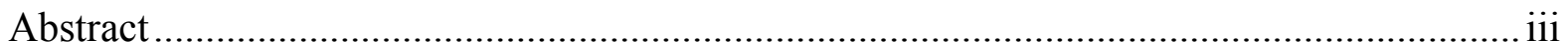

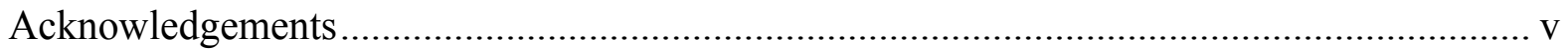

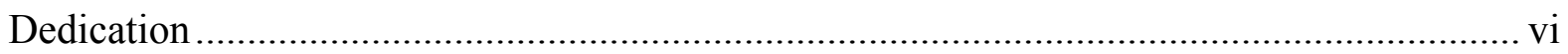

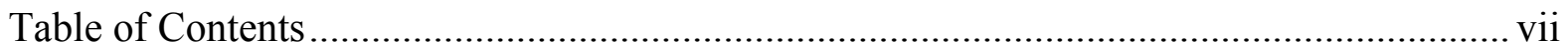

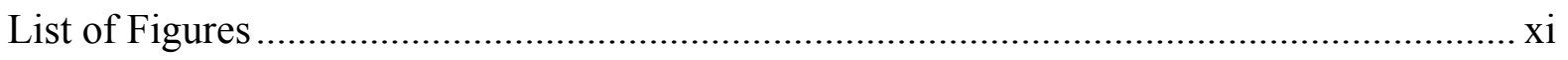

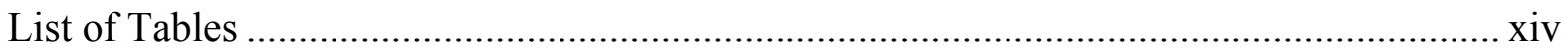

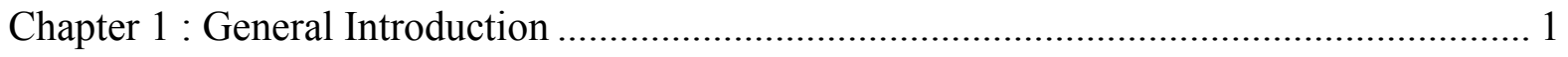

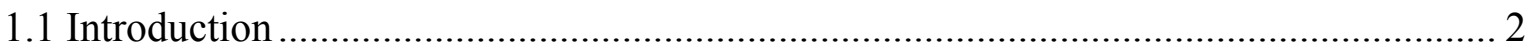

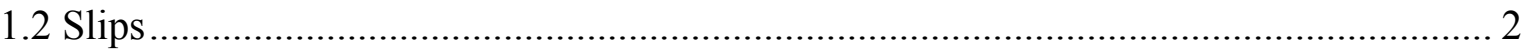

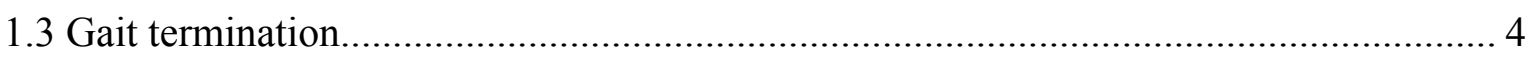

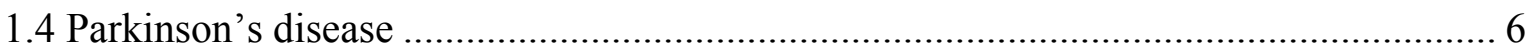

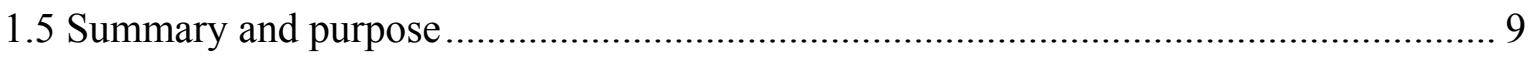

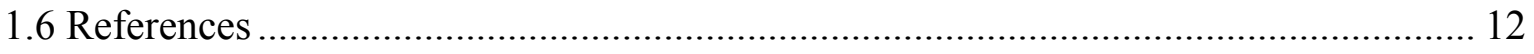

Chapter 2 : Control of Dynamic Stability during Gait Termination on a Slippery Surface* . 14

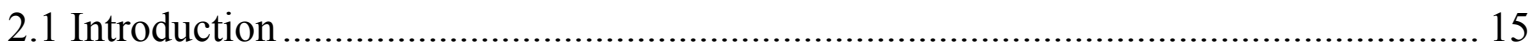

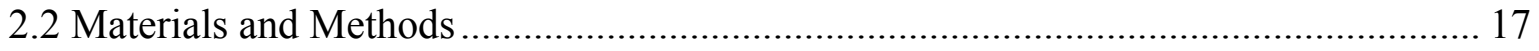

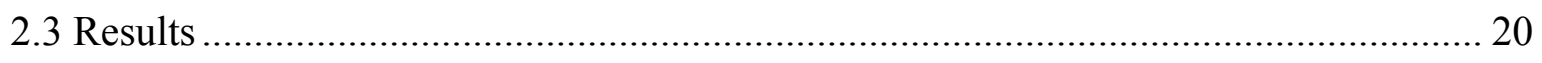

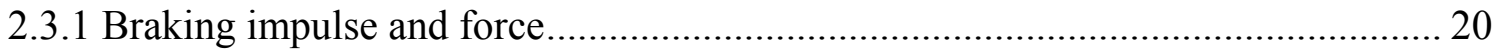

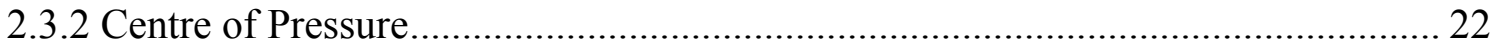


2.3.3 Step Parameters-Length and Width

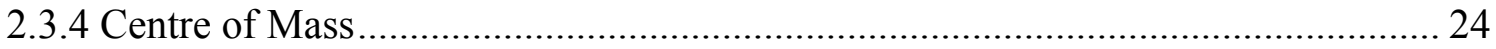

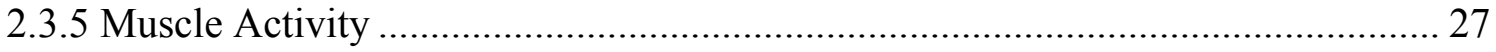

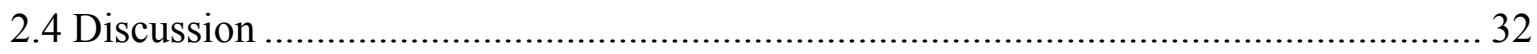

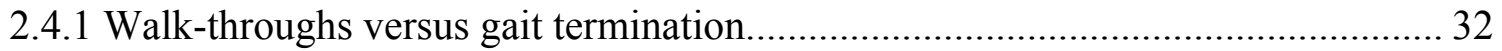

2.4.2 Gait termination on a normal surface versus a slippery surface ............................ 35

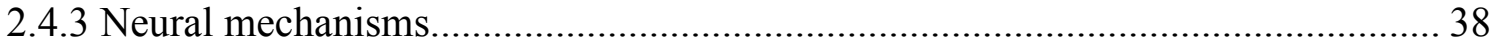

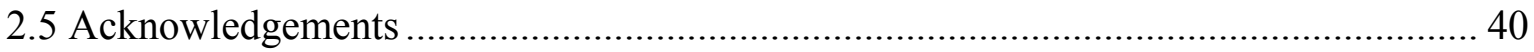

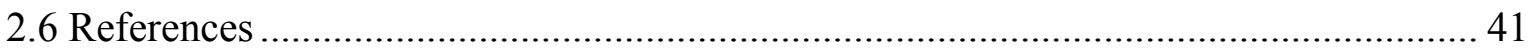

Chapter 3 : Adaptation to Gait Termination on a Slippery Surface in Young, Healthy Adults

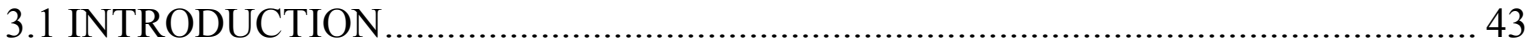

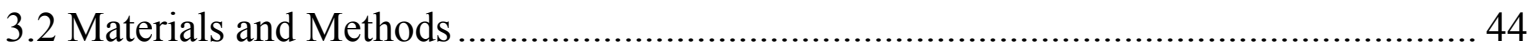

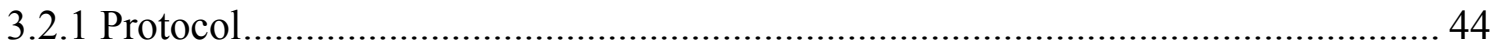

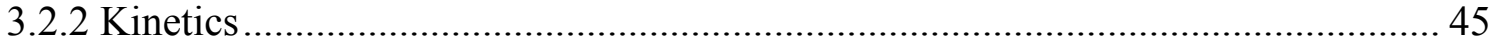

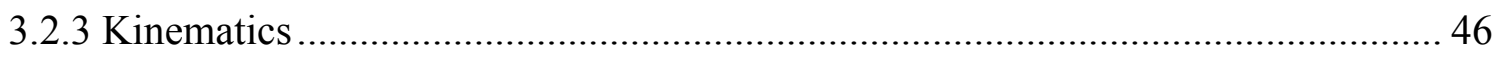

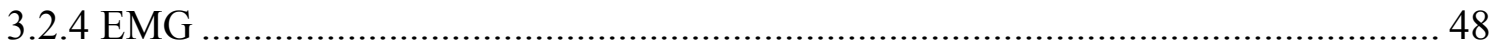

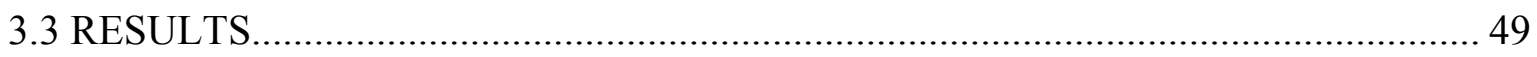

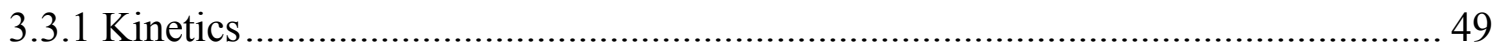

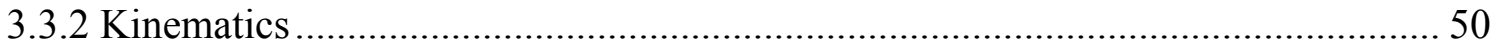

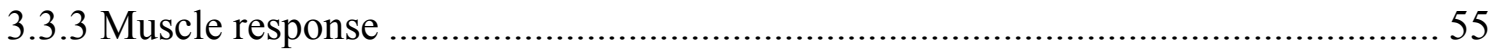




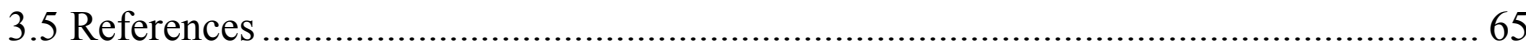

Chapter 4 : Control of Dynamic Stability during gait termination on a slippery surface with

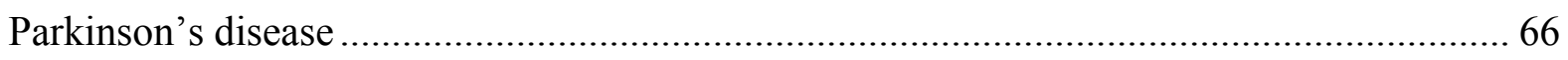

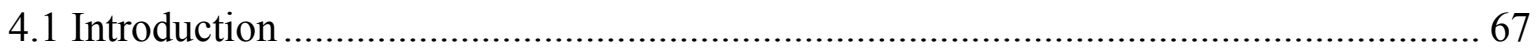

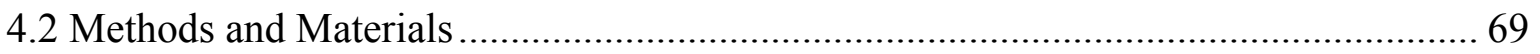

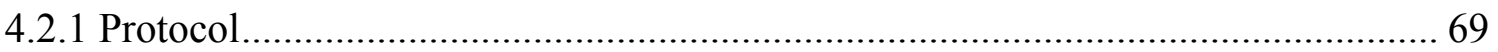

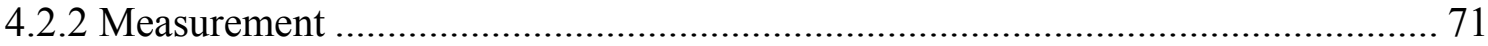

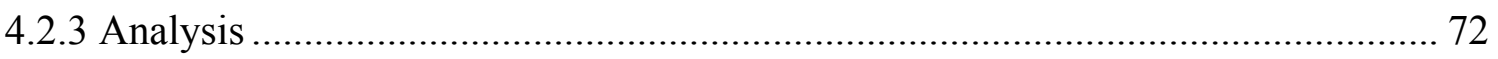

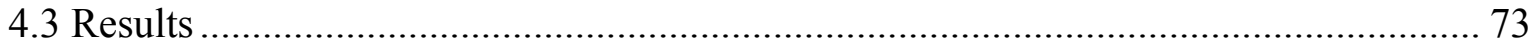

4.3.1 Planned versus Cued non-slippery stops .................................................. 73

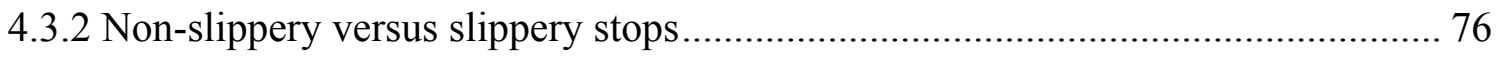

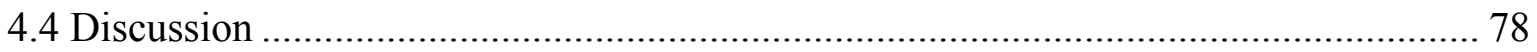

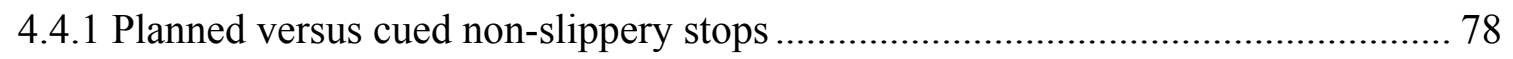

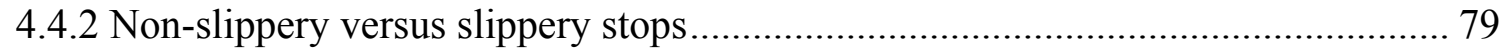

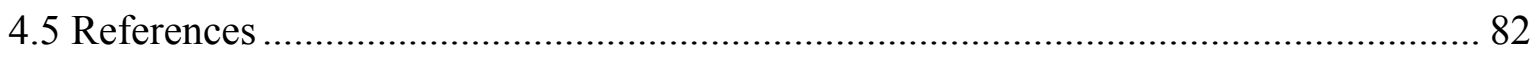

Chapter 5 : Adaptation to gait termination on a slippery surface with parkinson's disease... 83

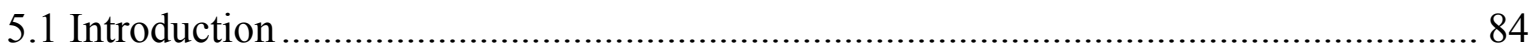

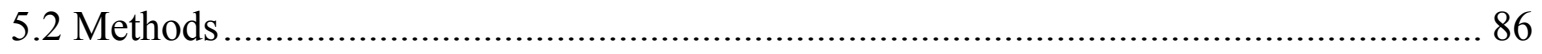

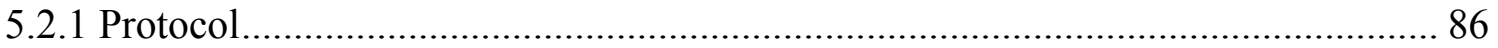

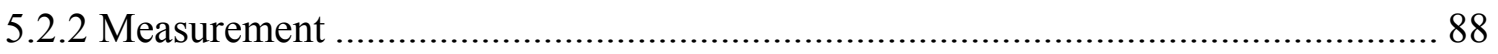


5.2.3 Analysis

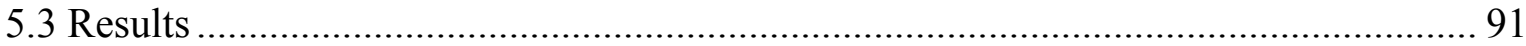

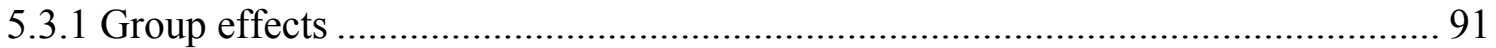

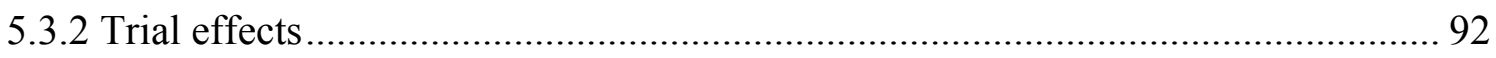

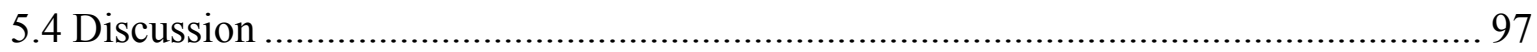

5.4.1 Integration of reactive and voluntary postural adjustments during gait termination

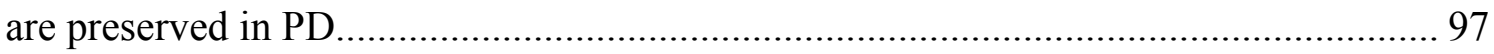

5.4.2 Adaptations to gait termination were observed for planned and cued slippery stops

5.5 References 104

Chapter 6 : General Discussion and Conclusions ...................................................... 106

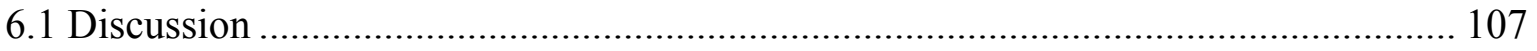

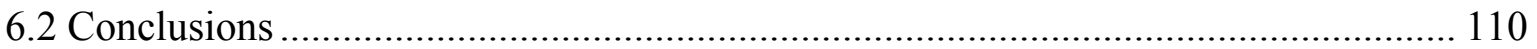

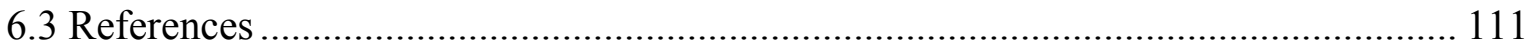

Appendix A Permission from the American Physiological Society ................................. 112 


\section{List of Figures}

Figure 2.1: Lab set-up diagram (not to scale) 16

Figure 2.2: A) Representative braking force profiles for walk-through (WT), non-slippery stop (NS), and slippery stop (SS) trials. Braking force (Fx) was recorded in the direction of progression. Zero seconds is heel contact on the rollers. ( $\mathrm{n}=8$ subjects).

B) Braking impulse (units $=$ N.s) and peak braking force averages (units $=N$ ) with SE for NS and SS trials. $*=$ significant difference between conditions $(\alpha<0.05) ; \mathrm{n}=8$ subjects.......21

Figure 2.3: A) Average root mean square (RMS) of center of pressure (COP) in the anteriorposterior (COPX) and medial-lateral (COPZ) axes with SE. B) Maximu $7 \mathrm{~m}$ range of COP with SE. * = significant difference between conditions $(\alpha<0.05, \mathrm{n}=8$ subjects $) \ldots \ldots \ldots \ldots \ldots . . .23$

Figure 2.4: A) Average COM trajectories in the sagittal plane. The dashed lines represent the same trials with the arms removed from the COM model. Position $(0,0)$ represents heel contact on the rollers. B) Average COM trajectories in the frontal plane. The dashed lines represent the same trials with the arms removed from the COM model. ( $n=6$ subjects for $A$ and $\mathrm{B})$.

Figure 2.5: A) Average COM momentum profiles. Vertical line indicates heel contact on the rollers. Solid lines represent SS trials, dashed lines represent NS trials. B) Comparison at peak vertical COM momentum during NS trials with SE. $*=$ significant difference between conditions $(\alpha<0.05) . \mathrm{n}=6$ subjects for $\mathrm{A}$ and $\mathrm{B}$ 26

Figure 2.6: Average peak COM momentum values compared between a full and an arm-less COM model with SE during SS trials. COMX and COMZ values were compared at maximum values, COMY values were compared at the minimum value. $*=$ significant difference between conditions. $(\alpha<0.05) . \mathrm{n}=6$ subjects.

Figure 2.7: Representative right limb EMG from one subject. Vertical line represents time of heel contact on the rollers. Horizontal axis is time (s). Signal to stop at 0s. Vertical axis is arbitrary units.

Figure 2.8: Representative left limb EMG from one subject. Vertical line represents time of heel contact on the rollers. Horizontal axis is time (s). Signal to stop at 0s. Vertical axis is arbitrary units

Figure 3.1: Diagram of laboratory set-up and display screens. Diagram is not to scale. ...... 47

Figure 3.2: Average braking force impulse (left vertical axis) and peak braking force (right vertical axis) (+/- SE). * indicates significant difference in the average braking impulse from that trial and the other trials indicated by the vertical lines. ${ }^{\wedge}$ indicates significant difference in the peak braking force from that trial and the other trials indicated by the vertical lines. (CNS = average of the cued non-slippery stop trials, CSS = cued slippery stop trials, $\mathrm{SS}=$ unexpected slippery stop trial). 
Figure 3.3: Average velocity one step before rollers (+/- SE). * indicates a significant difference between that trial and the other trials indicated by the vertical lines. (CNS $=$ average of the cued, non-slippery stop trials, CSS = cued slippery stop trials, SS = unexpected slippery stop trial)

Figure 3.4: Peak downward displacement of the COM (+/- SE). Values are normalized to the average value during walk-through trials. (CNS = average of the cued nonslippery stops, $\mathrm{SS}=$ unexpected slippery stop, CSS = cued slippery stop).

Figure 3.5: Average distance between the lead limb heel and the COM in the A-P direction at heel contact on the rollers $(+/-\mathrm{SE}) . *$ indicates a significant difference between that trial and other trials indicated by the vertical lines. (CNS = average of the cued non-slippery stops, SS = unexpected slippery stop, CSS = cued slippery stop)... 53

Figure 3.6: Foot angle at lead limb contact on the rollers (+/- SE). * indicates a significant difference between trials. (CNS = average of the cued non-slippery stops, $\mathrm{SS}=$ unexpected slippery stop, CSS = cued slippery stop)......

Figure 3.7: Peak arm raise height normalized to the unexpected slippery stop trial (SS, dashed line) $(+/-\mathrm{SE}) . *$ indicates a significant difference from the SS trial, $\wedge$ indicates a significant difference between trials. (CNS = average of the cued non-slippery stops, $\mathrm{SS}=$ unexpected slippery stop, CSS = cued slippery

stop).

Figure 3.8: Stacked plots of the average integrated EMG trends for each muscle in the lead (top plot) and trail (bottom plot) limb (+/- SE). (CNS = averagae of the cued nonslippery stops, $\mathrm{SS}=$ unexpected slippery stop, CSS = cued slippery stop).

Figure 4.1: Comparisons for step length and width for PD and control groups. The rectangles on the plots are the position of the force plates; in figure $\mathrm{D}$, the dashed rectangle is the final position of the moving right force plate. $(0,0)$ is the location of the lead heel contact on the force plate. The squares (dark are control, light are PD; error bars are SD) show the location of the left heel contact in A-C and right heel contact after the force plate has moved forward in $D$. The squares closest to $(0,0)$ are the terminal position of the left heel (A-C) or right heel (D). The lower squares in A-C are the location of the left heel contact preceding gait termination. * indicates a significant difference between groups (vertical line = significant difference in step length, horizontal line = significant difference in step width)

Figure 4.2: Dynamic stability during the first and second step of gait termination (+/$\mathrm{SE}) .{ }^{*}$ indicates a significant difference between groups or trials. The control group is represented by the dark bars, the PD group is represented by the light bars. (PNS = planned slippery stops, NS = cued non-slippery stops, SS = unexpected slippery stop) 
Figure 4.3: Forward COM velocity tracings showing time to slow and time to stop in representative control and PD participants. The triangle-shape represents time to slow, the circle represents time to stop. Time of zero seconds is heel contact on the force plate. The light lines represent the cued non-slippery stop trials, the dark lines represent the unexpected slippery stop trial. The shaded area is the time during which the platform moved forward. * indicates a significant difference of the group averages between conditions.

Figure 5.1: Average number of extra steps taken (+/- SE). ( SS = unexpected cued slippery stop, PSS = planned slippery stop, CSS = cued slippery stop).

Figure 5.2: Step length during the steps onto the force plate (lead limb) and subsequent, final step of gait termination (trail limb) $(+/-\mathrm{SE})$. The zero value represents placement of the right foot onto the force plate where the lead foot was in relation to the trail foot. * indicates a significant difference between that condition and the others indicated with a vertical line. (SS $=$ unexpected cued slippery stop, PSS = planned slippery stop, CSS = cued slippery stop) .. 94

Figure 5.3: Step width during the step onto the force plate (lead limb) and the subsequent, final step of gait termination (trail limb) $(+/-\mathrm{SE})$. The zero value indicates the position of the other foot during that step. * indicates a significant difference between that condition and the others indicated by the horizontal lines. ( $\mathrm{SS}=$ unexpected cued slippery stop, PSS = planned slippery stop, CSS = cued slippery stop) .

Figure 5.4: Average left arm raise for both groups normalized to unexpected cued slippery stop trial (+/-SE). * indicates a significant difference between that trial and the unexpected cued slippery stop trial (dashed line). (PSS = planned slippery stop, CSS = cued slippery stop) 


\section{List of Tables}

Table 2.1: Difference latencies comparing NS to WT and SS to NS trials. Values are means \pm SE. $\mathrm{n}=$ number of subjects who displayed significantly different muscle activation in $\geq 60 \%$ of NS trials compared to WT trials and the number of subjects who displayed significantly different muscle activation in slippery stop trial (SS) compared to NS trials. ${ }^{\wedge}=$ difference was inhibitory. Units are in ms

Table 3.1: Average iEMG values normalized to unexpected slippery stop trial (trial/SS trial) as shown in figure 3.8 .

Table 3.2: Summary table for dependent measures showing when adaptation occurred after the unexpected slippery stop trial (SS). Information in parentheses indicates when one trial was significantly different from the SS trial but the change did not persist across trials in that

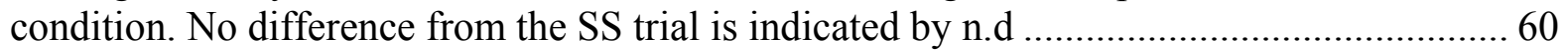

Table 4.1: Age, motor UPDRS score, and Hoehn and Yahr score for PD participants......... 69

Table 5.1: Age, motor UPDRS score, and Hoehn and Yahr score for PD participants......... 86

Table 5.2: Summary table for the independent measures showing when an adaptation occurred. The comparisons were with the unexpected slippery stop (SS). Information in parentheses indicates when one trial was different from the SS trial but the change did not persist across trials in that condition. No difference from the SS trial is indicated by n.d... 100 


\subsection{INTRODUCTION}

The focus of this thesis is the control of dynamic stability during gait termination (GT) on a slippery surface. Dynamic stability is the ability to control a moving centre of mass within a continually changing base of support. The ability of young and healthy individuals to maintain balance while stopping on an unexpectedly slippery surface and their subsequent adaptation to that surface reveals how the central nervous system (CNS) integrates a reactive, balance maintenance strategy into an on-going motor program. Stopping on a slippery surface also investigates the strategies involved to proactively prepare for the upcoming perturbation. A study involving participants with Parkinson's disease (PD) investigates how the CNS overcomes the dysfunctional basal ganglia to maintain balance and safely complete a stop. The differences between the participants with PD and the agematched controls will show the involvement of the basal ganglia in the GT motor program and the integration of a reactive strategy into a voluntary motor program. These investigations will provide more detail into the neural control of GT, the integration of a reactive response with proactive, voluntary movements and the importance of the basal ganglia sensorimotor integration abilities required for successful movement.

\subsection{SLIPS}

Falls and fall-related injuries can be very costly, both on a personal and a populationbased level. The loss of balance incurred by a slip has great potential to result in a fall or a fall-related injury (Cham and Redfern 2002). Understanding the mechanics which cause a 
slip as well as the strategies used to recover balance during a slip can provide useful information to develop prevention and training programs designed to decrease falls and fallrelated injuries.

Slip responses reflect the ability of the CNS and musculoskeletal system to reduce the potential of a slip in a dangerous environment and to recover balance through fall avoidance strategies (Cham and Redfern 2001 and 2002; Marigold and Patla 2002; Marigold et al. 2003). It is important to understand the relationship between gait and falls due to slippery surfaces (Cham and Redfern 2001). Defining the mechanical factors which determine slip outcomes can provide insight into the physiological variables which may limit the ability to prevent a fall resulting from the slip (Brady et al 2000; Cham and Redfern 2001).

Slip studies have typically been performed using steady-state gait. Slips have been induced using contaminated surfaces (e.g., Cham and Redfern 2001), a low-friction roller apparatus (e.g., Marigold and Patla 2002), or an anterior surface translation (e.g., Tang and Woollacott 1998). These studies reveal a slip response which includes a lowering of the COM (Marigold and Patla 2002; Cham and Redfern 2001), shortened subsequent step (Brady et al 2000; Cham and Redfern 2001; Marigold and Patla 2002; Tang and Woollacott 1998), an increased muscular response (Chambers and Cham 2006; Tang and Woollacott 1998; Tang and Woollacott 1999), and an arm elevation response (Tang and Woollacott 1998; Tang et al 1998; Marigold and Patla 2002). The CNS, using proprioceptive cues, responds to the slip in anywhere from $90 \mathrm{~ms}$ (Marigold and Patla 2002) to $250 \mathrm{~ms}$ (Marigold et al 2003) to 
generate a knee flexor and hip extensor moment (Cham and Redfern 2001; Chambers and Cham 2006) to support the upper body and regain balance.

Prior experience with and knowledge of a slippery surface dramatically changes how someone approaches and travels over the dangerous terrain. In anticipation of a slippery surface, step length decreases (Bhatt et al 2006; Brady et al 2000; Lockhart et al 2006), heel contact velocity and foot contact angle are reduced for a slower, flatter-footed landing (Brady et al 2000; Cham and Redfern 2002; Heiden et al 2006; Marigold and Patla 2002), and the strategy changes from purely reactive to proactive adaptations to diminish the destabilizing effects of the perturbation (Bhatt et al 2006; Chambers and Cham 2006; Marigold and Patla 2002). Muscle responses decrease as we rapidly learn how to safely and more efficiently maintain balance during the slip (Marigold and Patla 2002). People tend to adopt a more cautious gait (Cham and Redfern 2002; Chambers and Cham 2006; Heiden et al 2006) during the first few exposures to the slippery surface, but eventually become more confident and sometimes even "surf" over the slippery surface (Marigold and Patla 2002).

\subsection{GAIT TERMINATION}

Gait termination is gaining more attention as a complex, volitional transition between the repetitive, rhythmic motion of locomotion and quiet stance. Investigations of GT can illustrate how the nervous system prepares, controls and dissipates forward momentum of the body (Perry et al 2001). Studies of GT also assess the ability of the CNS to incorporate voluntary actions with a motor program not necessarily under voluntary control (Jaeger and 
Vanitchatchavan 1992). The ability of the CNS to integrate the various neural signals to smoothly and safely stop walking is the focus of the following GT studies.

During daily walking activity, when confronted with a large obstacle such as a car, person, boulder etc., our choice is either to change direction or stop walking. Both turning and GT require a decrease in forward momentum and, therefore, may be risk factors for falls in certain populations (e.g., elderly). Understanding how GT is achieved may help decrease the risk to those populations at risk of falling during transitional movements (Bishop et al 2004). Identifying strategies used in stopping may help with the etiology and mechanisms of types of falls in the elderly (Bishop et al 2004; Jaeger and Vanitchatchavan 1992) and also with gait training rehabilitation (Jaeger and Vanitchatchavan 1992).

The purpose of GT is to successfully stop ongoing locomotor rhythm by dissipating forward momentum to rest in a stable, upright standing posture. Gait termination has three basic requirements: 1) a decrease in the forward centre of mass (COM) momentum (Bishop et al 2004; Jian et al 1993; Perry et al. 2001); 2) control of foot placement to prepare the base of support (BOS) (Bishop et al. 2004; Perry et al. 2001); and 3) control of whole-body COM within the BOS limits (Bishop et al. 2004; Perry et al. 2001). The mechanics of GT which allow a person to stop successfully include an increased deceleration (braking) and decreased acceleration (push-off) through ground reaction forces (Hase and Stein 1998; Jaeger and Vanitchatchavan 1992). Most often, where and when we want to stop is known well before the task is required, but times arise when we need to stop quickly. If the signal to stop is provided within the first $30 \%$ of the gait cycle, most healthy people are able to safely and 
effectively stop within one step (Jaeger and Vanitchatchavan 1992). If the forward momentum is not sufficiently controlled, a person may take an extra step or a rise to toes, thereby transferring forward to vertical momentum (Hase and Stein 1998), to stop locomotion.

Gait termination on a slippery surface poses a more difficult balance task than locomotion over a slippery surface. The goal of GT is to cease COM movement and come to rest over a stationary BOS. A slippery surface removes the ability to use the ground reaction forces effectively and adds forward movement to the limb which first contacts the slippery surface. Examining GT on a slippery surface will show how the CNS maintains balance when perturbed during the transition from locomotion to quiet stance.

\subsection{PARKINSON'S DISEASE}

The basal ganglia are an important group of nuclei which function to regulate movement. Specifically, the basal ganglia are involved in managing centrally initiated movements (Bloem and Bhatia 1004; Morris et al 2001), adapting behaviour to changing environmental conditions (Bloem and Bhatia 2004; Dimitrova et al 2004; Frank et al 2000; Morris 2006), and controlling output gain for movements (Bloem and Bhatia 2004; Dimitrova et al 2004). Parkinson's disease is caused by the loss of dopamine producing cells within the basal ganglia (Bloem and Bhatia 2004) which results in a movement disorder. The cardinal features of PD are bradykinesia, resting tremor, rigidity, and postural instability (Jankovic and Stacy 1999). PD provides a unique insight into the importance of the basal ganglia and their dopaminergic pathways in the control of everyday movement. PD also 
illustrates how the basal ganglia are involved in and how PD affects the ability to integrate a reactive balance-correcting response into the voluntary movement of gait termination.

Typical Parkinsonian gait includes a stiff, shuffling, slow walk which can often include festination when attempting to stop (Bloem and Bhatia 2004; Morris 2006; Morris et al 2001). People with PD often exhibit a lack of arm swing, a forward stooped posture and a general decrease in movement amplitude seen in a decreased toe clearance, step length, and trunk rotation (Carpenter et al 2004; Morris 2006; Morris et al 2001). Traditional medical treatment, dopamine replacement therapy, helps increase speed and stride-length in gait but do not change the rhythmicity of locomotion (Morris 2006; Morris et al 2001; Vaillancourt et al 2004).

A central or voluntary movement requires the release and cessation of a motor program for accurate movement. The increased inhibitory basal ganglia projections to the thalamo-cortical motor circuit in PD (Delong 2000) causes difficulties initiating and terminating voluntary movements and also switching between motor programs (Morris 2006). Previous studies of GT in persons with PD have shown that the GT strategy used by PD subjects relies more on a decrease in acceleration rather than an increase in braking. This behavior does not change with delayed timing of the gait termination cue (Bishop et al 2003) which suggests that GT is operated as a separate program and, therefore, involves the common initiation problems that people with PD often experience initiating gait (Abbruzzese and Berardelli 2003; Halliday et al 1998; Morris 2006; Morris et al 2001; Nieuwboer et al 2004). 
Gait termination on an unexpectedly slippery or moving surface requires the use of a purely reactive strategy to maintain balance and safely stop walking. People with PD often experience a 'kinesia paradoxica' (Bloem and Bhatia 2004) where a quick response to an environmental event using unexpectedly good movement ensues. A normal or even faster reactive response to perturbations is often recorded despite the hypokinetic force production and often inaccurate behavioural output (Carpenter et al. 2004; Dimitrova et al 2004; Horak et al 1996; Morris et al 2001; Nieuwboer et al 2004). The instability seen in PD is not corrected with medication and is especially apparent for external perturbations (Carpenter et al 2004; Frank et al 2000; Horak et al 1996; Morris 2006); therefore, traditional levodopa therapy does not provide protection against slips and falls due to involuntary balance disturbances. In fact, a majority of falls in PD occur indoors and during the day during peak activity and medication levels (Bloem and Bhatia 2004). Testing people with PD while on their optimal medication may provide insight into why these falls occur.

Unexpected, cued GT requires a change in central set or motor program from repetitive locomotion to quiet stance within one stride. Alternatively, preplanned GT allows appropriate preparations from the beginning of the task. People with PD often have difficulty switching central set (Bishop et al 2003; Dimitrova et al 2004; Frank et al 2000; Morris 2006; Tang and Woollacott 1999) which would make cued GT more difficult than preplanned GT. Both tasks, however, require a change in motor programs and may pose an equally great challenge for someone with PD. 
Cued GT, in this thesis, involves a visual trigger. People with PD often rely on external sensory information for movement initiation and execution (Abbruzzese and Berardelli 2003; Morris 2006; Morris et al 2001). While the basal ganglia are not properly controlling voluntary movement in PD, the CNS attempts to use more lateral, associated brain areas such as the premotor and parietal cortices, and the cerebellum to compensate for BG dysfunction to increase the size and speed of bradykinetic movements (Berardelli et al 2003; Hanakawa et al 1999; Morris 2006; Morris et al 2001). These parallel pathways of processing may allow for easier movement when the visual trigger is used.

Sensory gating and sensorimotor integration is another suggested function of the basal ganglia (Abbruzzese and Berardelli 2003; Berardelli et al 2003). Abnormal scaling of sensory input may be responsible for any inaccuracies in PD movements. Consequently, when the CNS 'bypasses' the basal ganglia using parallel pathways, it may be attempting to use alternate sensorimotor integration areas to properly manage and effectively use the ascending sensory information to accurately control voluntary movement. This extraneous use of cortical power, however, is suggested to saturate the processing limits which may explain why PD have difficulty switching tasks and/or performing more than one task at a time (Berardelli et al 2003).

\subsection{SUMMARY AND PURPOSE}

Gait termination on slippery surface is a two-fold challenge: First, a voluntary gait termination program must be initiated and, second, a reactive balance-correcting program must be generated and integrated into the on-going gait termination program. This 
dissertation presents four studies which examine the following questions about the challenges of stopping on a slippery surface: First, how does a young, healthy adult integrate a slip response into a gait termination program? Is the slip response during gait termination similar to that seen in locomotion or does it differ because of the increased demands of gait termination over locomotion? The second study examines how experience with and knowledge of the slippery surface changes the behaviour during a slippery stop. How quickly does an adaptation occur in young, healthy adults? Is the adaptation in gait termination similar to that seen in slips during locomotion or does the added challenge of stopping change the adaptive behaviour?

Parkinson's disease provides a model of the involvement of the basal ganglia in voluntary movements. Research suggests that PD affects the ability to initiate motor programs, switch between motor tasks and, even when on medication, maintain balance during external perturbations. Gait termination is a voluntary movement that must be initiated during locomotion; therefore, the third study asks how the basal ganglia are involved in switching to gait termination. Also does the involvement of the basal ganglia differ between planned and cued gait termination? How will Parkinson's disease affect the ability to integrate a balance-correcting response into the voluntary gait termination? Finally, the fourth study examines the effect of PD on the adaptation to a slippery surface. How quickly does an adaptation occur? Is the adaptation similar that seen to young, healthy adults? This dissertation will describe how the CNS integrates reactive and voluntary movements and, using Parkinson's disease to investigate the function of the basal ganglia, how the basal 
ganglia are involved in switching locomotor tasks, integrating a balance-correcting response into a voluntary movement, and adapting to repeated exposure to a slippery surface. The results of this dissertation can be used, at a theoretical level, to understand CNS and basal ganglia function during locomotor tasks and during perturbations to locomotor tasks and, at an applied level, to generate programs to decrease the incidence of falls related to slipping in both the workplace and the community for people of all ages. 


\subsection{REFERENCES}

Abbruzzese G, and Berardelli A. (2003). Research Review: Sensorimotor integration in movement disorders. Movement Disorders, 18(3): 231-240.

Berardelli A, Rothwell JC, Thompson PD, and Hallett M. (2001). Review article: Pathophysiology of bradykinesia in Parkinson's disease. Brain, 124: 2131-2146.

Bhatt T, Wang E, and Pai Y-C. (2006). Retention of adaptive control over varying intervals: Prevention of slipinduced backward balance loss during gait. J Neurophysiol, 95: 2913-2922.

Bishop MD, Brunt D, Kukulka C, Tillman, MC, and Pathare N. (2003). Braking impulse and muscle activation during unplanned gait termination in human subjects with Parkinsonism. Neurosci Letters, 348: 89-92.

Bishop M, Brunt D, Pathare N, and Patel B. (2004). The effect of velocity on the strategies used during gait termination. Gait Posture, 20: 134-139.

Bloem BR and Bhatia KP. (2004). Gait and balance in basal ganglia disorders. In Clinical Disorders of Balance, Posture and Gait ( $2^{\text {nd }}$ Edition). Eds. Bronstein AM, Brandt T, Woollacott MH, and Nutt JG: 173-206.

Brady RA, Pavol MJ, Owings TM and Grabiner MD. (2000). Foot displacement but not velocity predicts the outcome of a slip induced in young subjects while walking. J Biomech, 33: 803-808, 2000.

Carpenter MG, Allum JHJ, Honegger F, Adkin AL, and Bloem BR. (2004). Postural abnormalities to multidirectional stance perturbations in Parkinson's disease. J Neurol Neurosurg Psychiatry, 75: 1245-1254.

Cham R, and Redfern MS. (2001). Lower extremity corrective reactions to slip events. J Biomech, 34: 14391445.

Cham R, and Redfern MS. (2002). Changes in gait when anticipating slippery floors. Gait Posture, 15: 159-171.

Chambers AJ, and Cham R. (2006). Slip-related muscle activation patterns in the stance leg during walking. Gait Posture, doi: 10.1016/j.gaitpost.2006.06.007.

Crenna P, Cuong DM, and Brénière Y. (2001). Motor programmes for the termination of gait in humans: organization and velocity-dependent adaptations. J Physiol, 537.3: 1059-1072.

Delong, M.R. (2000). The basal ganglia. In Principles of Neural Science ( $4^{\text {th }}$ Edition). Eds. Kandel, E.R., Schwartz, J.H., \& Jessell, T. M: 853-867.

Dimitrova D, Horak FB, and Nutt JG. (2004). Postural muscle responses to multidirectional translations in patients with Parkinson's disease. J Neurophysiol, 91: 489-501.

Frank JS, Horak FB, and Nutt J. (2000). Centrally initiated postural adjustments in Parkinsonian patients on and off levodopa. J Neurophysiol, 84: 2440-2448.

Halliday SE, Winter DA, Frank JS, Patla AE, and Prince F. (1998). The initiation of gait in young, elderly, and Parkinson's disease subjects. Gait Posture, 8: 8-14.

Hanakawa T, Katsumi Y, Fukuyama H, Honda M, Hayashi T, Kimura J, and Shibasaki H. (1999). Mechanisms underlying gait disturbance in Parkinson's disease. A single photon emission computer tomography study. Brain, 122: 1271-1282.

Hase K, and Stein RB. (1998). Analysis of rapid stopping during human walking. J Neurophysiol, 80: 255-261.

Heiden TL, Sanderson DJ, Inglis JT, and Siegmund GP. (2006). Adaptations to normal human gait on potentially slippery surfaces: The effects of awareness and prior slip experience. Gait Posture, 24:237-246. 
Horak FB, Frank J, and Nutt J. (1996). Effects of dopamine on postural control in Parkinsonian subjects: Scaling, set, and tone. J Neurophysiol, 75(6): 2380-2396.

Jaeger R, and Vanitchatchavan P. (1992). Ground reaction forces during termination of human gait. J Biomech, 25 (10): 1233-1236.

Jankovic J, and Stacy M. (1999). Movement Disorders. In Textbook of Clinical Neurology. Eds. Goetz CG and Pappert EJ: 655-664.

Jian Y, Winter DA, Ishac MG, and Gilchrist L. (1993). Trajectory of the body COG and COP during initiation and termination of gait. Gait Posture, 1: 9-22.

Lockhart TE, Spaulding JM, and Park SH. (2006). Age-related slip avoidance strategy while walking over a known slippery floor surface. Gait Posture, doi:10.1016/j.gaitpost.2006.08.009.

Marigold DS, and Patla AE. (2002). Strategies for dynamic stability during locomotion on a slippery surface: effects of prior experience with and knowledge about surface characteristics. J Neurophysiol, 88: 339-353.

Marigold DS, Bethune AJ, and Patla AE. (2003). Role of the unperturbed limb and arms in the reactive recovery response to an unexpected slip during locomotion. J Neurophysiol, 89: 1727-1737.

Morris ME. (2006). Locomotor training in people with Parkinson disease. Physical Therapy, 86(10): 14261435.

Morris ME, Huxham F, McGinley J, Dodd K, and Iansek R. (2001). Review: The biomechanics and motor control of gait in Parkinson disease. Clin Biomech, 16: 459-470.

Nieuwboer A, Dom R, De Weerdt W, Desloovere K, Janssens L, and Vangheluwe S. (2004). Electromyographic profiles of gait prior to onset of freezing episodes in patients with Parkinson's disease. Brain, 127: 1650-1660.

Perry SD, Santos LC, and Patla AE. (2001). Contribution of vision and cutaneous sensation to the control of centre of mass (COM) during gait termination. Brain Res,913: 27-34.

Tang PF and Woollacott MH. (1998). Inefficient postural responses to unexpected slips during walking in older adults. J Gerontol: Med Sci, 53A: M471-M480.

Tang PF, Woollacott MH, and Chong RKY. (1998). Control of reactive balance adjustments in perturbed human walking: roles of proximal and distal postural muscle activity. Exp Brain Res, 119: 141-152.

Tang PF and Woollacott MH. (1999). Phase-dependent modulation of proximal and distal responses to slips in young and older adults. J Gerontology: Med Sci, 54A (2): M89-M102.

Vaillancourt DE, Prodoehl J, Metman LV, Bakay RA, and Corcos DM. Effects of deep brain stimulation and medication on bradykinesia and muscle activation in Parkinson's disease. Brain, 127: 491-504. 


\section{CHAPTER 2: CONTROL OF DYNAMIC STABILITY DURING GAIT TERMINATION ON A SLIPPERY SURFACE*}

Please see Appendix A for permission to use the previously published manuscript. * Used with permission from the American Physiological Society. 


\subsection{INTRODUCTION}

Gait termination (GT) is defined as the transient period from repetitive gait to a full stop (Jian et al. 1993). Stopping is a great challenge to the body as the nervous system must effectively change the body from a dynamic to a static state. Three common ways by which to successfully terminate gait include: decreased acceleration of whole-body centre of mass (COM) through a flexor synergy in the hind leg (Hase and Stein 1998; Jaeger and Vanitchatchavan 1992), increased deceleration of whole-body COM through an extensor synergy in the front limb (Hase and Stein 1998; Jaeger and Vanitchatchavan 1992), and an energy/momentum transfer through a toe raise (Hase and Stein, 1998) or a momentum transfer to another plane of movement (O'Kane et al. 2003).

Muscle synergies employed by the central nervous system (CNS) manipulate limb movement to alter the centre of pressure (COP) beneath the feet. The COP controls the COM during gait termination (Jian et al. 1993) and can influence COM position in three ways (B McGowan unpublished observations): First, a change in foot placement, such as an increase in step length, will move the COP ahead of the COM and increase the ability to provide a braking force. Second, limb loading/unloading strategies can also be used to control the COP/COM. A flexor synergy in the limb taking the final step before termination unloads that limb thereby decreasing the acceleration and lowering the COM. Loading the limbs in the final stance phase, an extensor synergy, increases the braking force and decelerates the COM (Hase and Stein, 1998). Limb loading/extension can also raise or shift the COM, thereby converting some kinetic energy to potential energy or transferring momentum to another 
plane in order to dissipate forward momentum (O'Kane et al. 2003). Finally, the excursions of the COP within the base of support (BOS) can influence the COM position and assist gait termination.

A slippery surface removes the ability to effectively manipulate the COP and eliminates a large part of COM-control during movement. Previous studies investigating slip responses during gait report a rapid onset of a flexor synergy to lower the COM and improve stability as well as arm elevation to stabilize the unexpected COM displacement (Cham and Redfern 2001, 2002; Marigold and Patla 2002; Marigold et al. 2003). The purpose of the present study was to examine the response to an unexpected slip during gait termination. Slip responses during gait termination are expected to parallel those measured in steady-state gait but increase in magnitude as the loss of COP control during gait termination is hypothesized to be more detrimental than during steady-state gait.

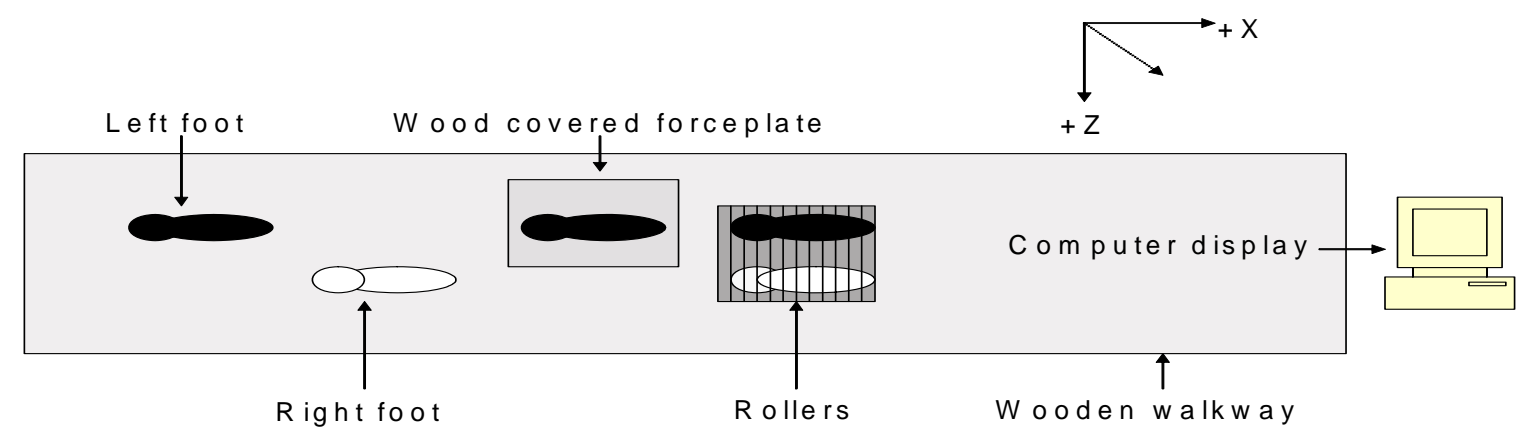

Figure 2.1: Lab set-up diagram (not to scale). 


\subsection{MATERIALS AND METHODS}

Gait termination (GT), on a normal and a slippery surface, was investigated in eight healthy, young adults [ 4 males and 4 females, age $=24,+/-3(\mathrm{SD})$ yr]. Experimental set-up included a four-inch high wooden walkway with aluminum rollers covering one force plate (See Marigold and Patla, (2002) for detailed description of the roller apparatus). A set of ten walk-through (WT) trials were performed along the walkway to collect baseline gait data. The subject then performed a series of 21 trials, of which a random five out of the first 20 trials required termination on a set of locked rollers [non-slippery stop (NS) trials]. As the subject stepped with the left foot on the first force plate (covered by wood), a monitor at the end of the walkway signaled for the subject to stop with both feet on the second force plate (covered by the rollers) (Figure 2.1). The $21^{\text {st }}$ trial always signaled the subject to stop. For the $21^{\text {st }}$ trial, however, the rollers were unlocked without the subject's knowledge to create a slippery surface [slippery stop (SS) trials] and therefore a purely unexpected slip trial. The rollers were aligned so the slip was only in the direction of progression (along the $\mathrm{x}$-axis). The protocol was approved by the University of Waterloo Ethics committee and safety measures were taken to ensure subjects did not fall during SS trials.

Two AMTI $^{\odot}$ force plates were used to collect ground reaction forces sampled at $2400 \mathrm{~Hz}$. Previous testing (A Oates unpublished observations) of the force plates with the wooden platform and roller coverings demonstrated accurate (within 2\%) data recording, with the exception of the natural frequency and the sensitivity of the system. These system 
differences were deemed acceptable for this experiment. Force plate data were low-pass filtered at $60 \mathrm{~Hz}$ and analyzed using a custom program.

Ground reaction forces represent the algebraic sum of the mass-acceleration products of all segments in the body and therefore provide a representation of COM control. Braking impulse was calculated as the integration of the horizontal ground reaction force in the direction of progression over a set time period. The integration period was determined by the length of foot contact on the rollers during the SS trials. This integration period was constant for a subject but varied between subjects according to the length of foot contact by each subject on the slippery surface (range $=0.12$ to 0.35 seconds). The braking impulse and the peak braking force were compared between NS and SS conditions. The average range of COP movement [calculated as the root-mean-square (RMS) of all deviations in the $\mathrm{x}-$ and $\mathrm{z}-$ axis] as well as the maximum range of COP was compared between all conditions.

Three Optotrak@ (Northern Digital Inc., Waterloo) cameras recorded kinematic data from 21 infra-red emitting diodes (IREDs) at $60 \mathrm{~Hz}$ to create a 12 -segment model for COM calculation. IREDs were placed at: xyphoid process and bilaterally at fifth metatarsal, heel, lateral malleolus, lateral femoral condyle, greater trochanter, ASIS, acromion process, olecranon, radial styloid, medial clavicle. Raw data was processed using Optofix and CofM software (MIshac Kinetics) and low-pass filtered at 6 Hz. Step length and width values were measured from the heel markers at the point of heel contact on the force plates. To calculate the contribution of the arm segments to the slip-recovery response, the arms were removed from the COM model to create a 10-segment COM model. The resulting arm-less COM peak 
displacements and peak momentum profiles were compared to those with arms. (Note: Arm marker data was missing for two subjects. These two subjects, therefore, were not included in the comparison between the full COM model and the arm-less COM model). Final COM position in the $x$ axis, the peak displacements of the COM in the vertical ( $y$ axis) and horizontal ( $z$ axis) were calculated both with and without arms segments for the gait termination trials. COM momentum profiles were compared between NS and SS trials to identify any momentum transfers during GT. Instantaneous COM momentum values were compared at peak values of the NS trials after heel contact on the rollers.

Muscle activity was recorded from 10 pairs of bipolar Kendall Meditrace (Chicopee, MA) surface electrodes: tibialis anterior (TA), soleus (SOL), rectus femoris (RF), biceps femoris (BF), and lower erector spinae (LES), bilaterally) sampled at $2400 \mathrm{~Hz}(0.5-1 \mathrm{~K}$ gain with a Bortec ${ }^{\odot}$ AMT-8 amplifier. EMG data was analyzed using a custom-designed Matlab program (Mathworks, Natick, MA) which full-wave rectified, filtered $\left(2^{\text {nd }}\right.$ order Butterworth with a low pass frequency cut-off of $10 \mathrm{~Hz}$ ) and aligned the data with heel contact on the force plates. For comparison purposes, WT trials and NS trials were compared by aligning the data to heel contact on the first force plate. NS and SS trials were compared by aligning the data to heel contact on the rollers. These alignment points were chosen to illustrate any differences in behaviour that accompanied the differences due to surface conditions.

Significant muscle activity changes (defined as a change beyond two standard deviations of the average muscle activity in the one second before heel contact with a minimum duration of 50ms) were determined and difference latencies (average WT trials 
subtracted from average NS trials and average NS trials subtracted from SS trials) were calculated (Marigold and Patla 2002). The differences between WT and NS trials will be referred to as latencies whereas the differences between NS and SS trials will be referred to as difference latencies. When comparing the WT and NS trials, a significant change in activity is reported only when that muscle showed significantly different activity in $60 \%$ of the NS trials. Pre-planned comparisons, using paired t-tests, were conducted for WT versus NS $(\alpha=0.05)$. Non-parametric Wilcoxon tests were conducted for the NS versus SS trials because of the differences in sample size $(n=35$ versus $n=8, \alpha=0.05)$.

\subsection{RESULTS}

Subjects were able to stop successfully on the rollers in 35/40 NS trials. In two unsuccessful NS trials (one trial each for two subjects), the subject reported that he/she was not paying attention to the monitor at the end of the walkway. For the remaining three unsuccessful trials (all within the same subject), the subject seemed to be walking too fast to stop with both feet on the rollers. These five unsuccessful NS trials were not included in the analysis. All subjects were able to regain balance and eventually stop safely (i.e., without falling or requiring assistance to maintain balance) during the SS trials.

\subsubsection{Braking impulse and force}

Braking force illustrates the amount of force generated to stop forward movement. Subjects exerted a significantly lower braking impulse during SS trials compared to NS trials $(\mathrm{p}=0.0120)$ (Figure $2.2 \mathrm{~A}$ and B). The peak braking force, however, did not differ 
significantly between gait termination conditions (Figure 2.2B). The slippery surface prevented the subjects from developing a sufficient braking impulse to stop forward progression.
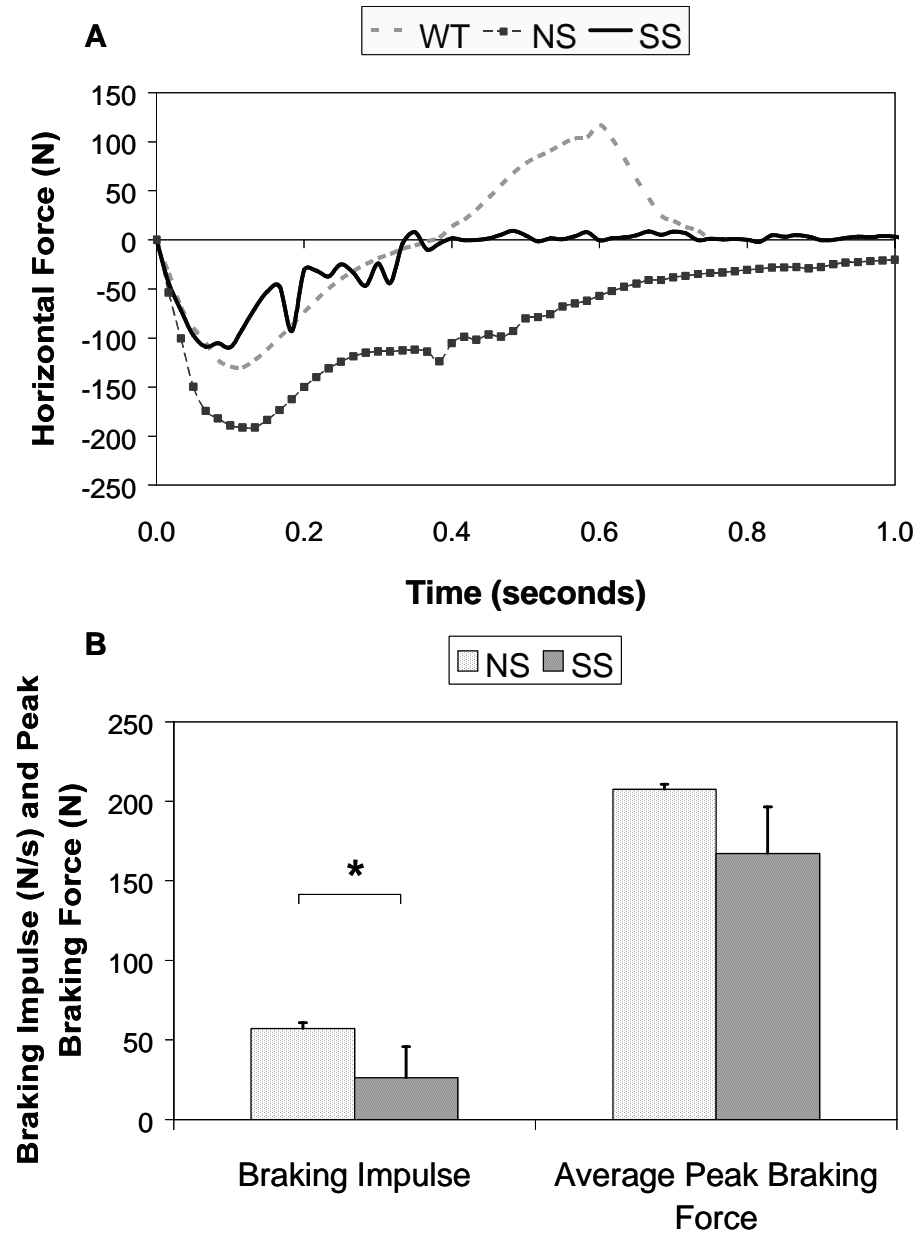

Figure 2.2: A) Representative braking force profiles for walk-through (WT), nonslippery stop (NS), and slippery stop (SS) trials. Braking force (Fx) was recorded in the direction of progression. Zero seconds is heel contact on the rollers. ( $n=8$ subjects). B) Braking impulse (units $=$ N.s) and peak braking force averages $($ units $=N$ ) with $\mathrm{SE}$ for NS and SS trials. * = significant difference between conditions $(\alpha<0.05) ; \mathrm{n}=8$ subjects. 


\subsubsection{Centre of Pressure}

The RMS of the COP in all directions differed significantly between both WT and NS and NS and SS conditions (Figure 2.3A). In the direction of progression (COPX), the RMS was significantly larger in the WT trials compared to the NS trials $(\mathrm{p}=0.0227)$ but there was no difference in the maximum range of COPX values between the WT and NS conditions $(\mathrm{p}=0.9009$; Figure 2.3B). The increased deviation is expected as the COP travels under the entire length of the foot as the subjects continue walking past the force plate. Subjects likely used their entire plantar surface to move the COP during both static foot placements of WT and NS trials; a likely explanation for the lack of difference between the maximum range of COPX movement between the WT and NS trials. In the medial-lateral plane (COPZ), both the RMS (Figure 2.3A) and maximum range (Figure 2.3B) values were significantly greater in the NS trials than the WT trials ( $\mathrm{p}=0.0002$ for RMS and $\mathrm{p}=0.0011$ for maximum range). As both feet are placed on the force plate during gait termination, the COP has a greater area within to move and therefore the increased deviations and ranges of COP values are expected when comparing NS to WT trials.

A comparison between the NS and SS trials reveals an increase in all four variables during the slip (Figure 2.3, A and B): RMS, $\mathrm{p}=0.0120$ for COPX and $\mathrm{p}=0.0120$ for COPZ; maximum range, $\mathrm{p}=0.0170$ for COPX and $\mathrm{p}=0.0120$ for COPZ. The increased deviations and ranges indicate the foot was not able to remain static on the slippery surface as the foot slid forward. Subjects were therefore not able to stop successfully with the same COP pattern. 

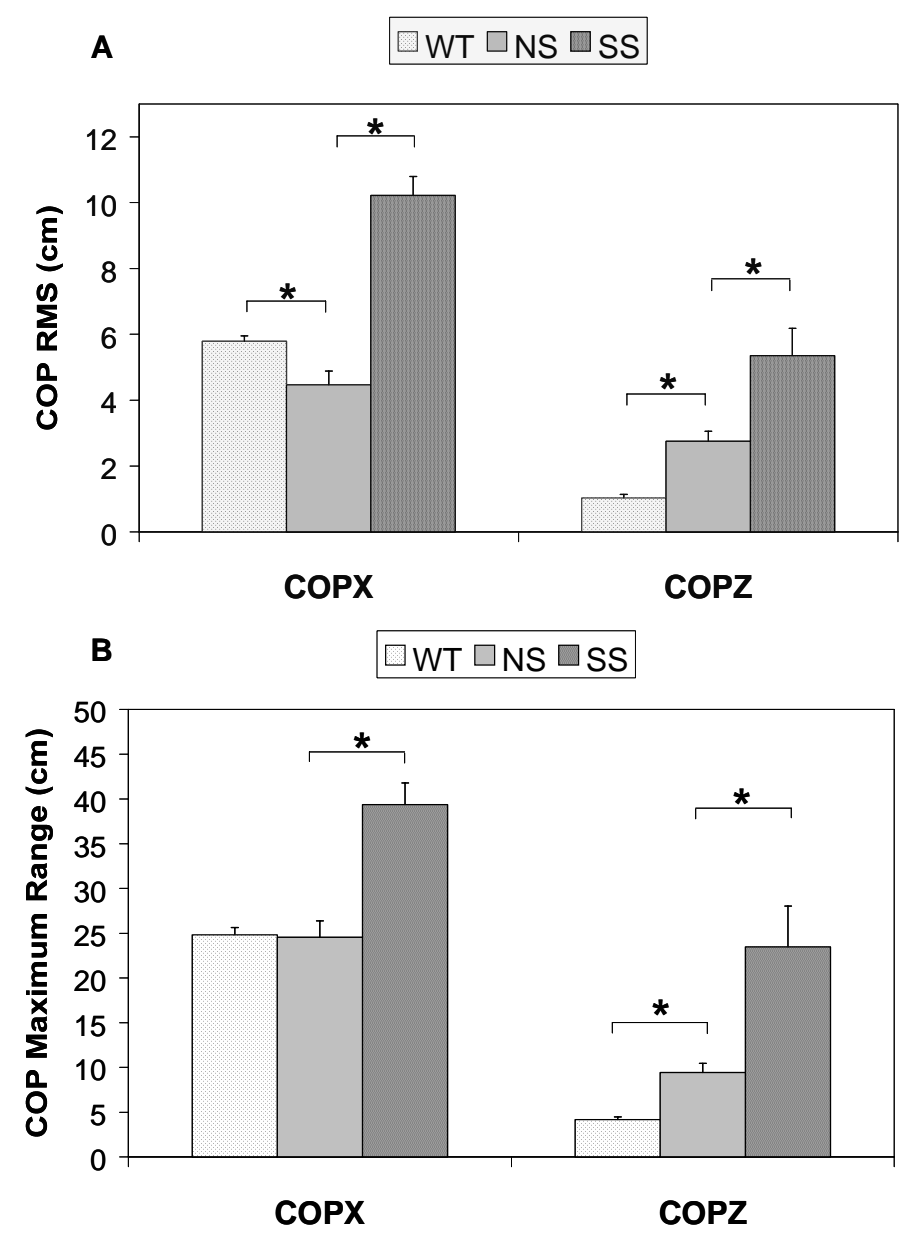

Figure 2.3: A) Average root mean square (RMS) of center of pressure (COP) in the anterior-posterior (COPX) and medial-lateral (COPZ) axes with SE. B) Maximu7m range of COP with SE. * = significant difference between conditions $(\alpha<0.05, n=8$ subjects).

\subsubsection{Step Parameters-Length and Width}

Average step length was $80.8+/-5.3$ (SD) cm for WT trials, 80.3(+/-3.4 cm for NS trials and $82.2+/-3.6 \mathrm{~cm}$ for SS trials. Average step width was $26.6+/-2.8 \mathrm{~cm}$ for WT trials, $28.1+/-3.7 \mathrm{~cm}$ for NS trials and 28.3+/-2.9 cm for SS trials. Unlike previous investigations (B McGowan, unpublished observations) there was no significant difference between WT and NS trials in either step length or width. The lack of difference between the WT and NS conditions suggests that subjects did not attempt to use changes in step parameters to 


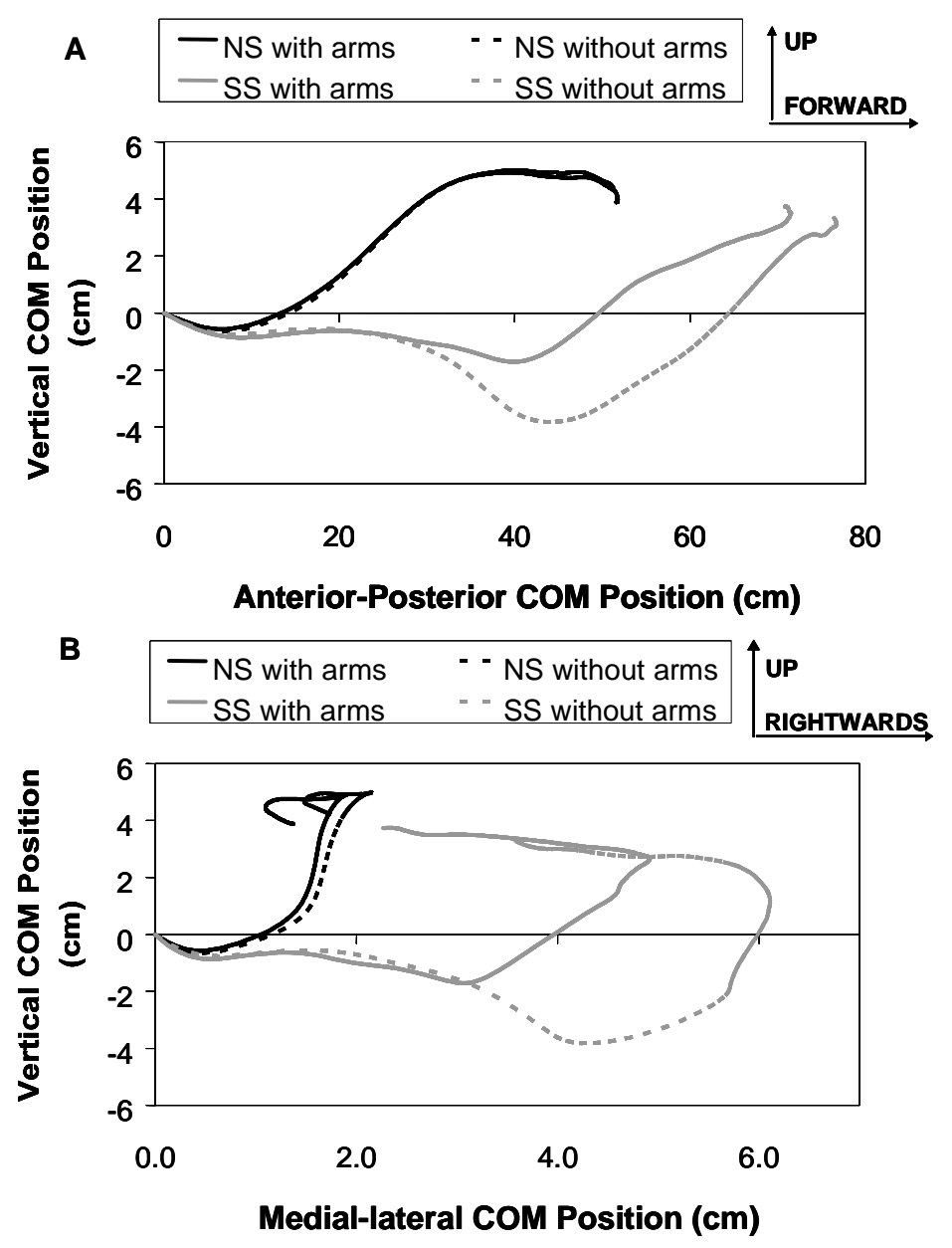

Figure 2.4: A) Average COM trajectories in the sagittal plane. The dashed lines represent the same trials with the arms removed from the COM model. Position $(0,0)$ represents heel contact on the rollers. B) Average COM trajectories in the frontal plane. The dashed lines represent the same trials with the arms removed from the COM model. $(\mathrm{n}=6$ subjects for $\mathrm{A}$ and $\mathrm{B})$.

terminate locomotion. There were also no differences in step parameters between the NS and SS trials which confirms that subjects were unable to anticipate the slippery surface.

\subsubsection{Centre of Mass}

The COM moved significantly more in all three planes during the SS compared to the

NS trials both with and without arms (Figure 2.4, A and B). In the direction of progression, 
the COM moved on average $51.6+/-7.7 \mathrm{~cm}$ with arms in the NS trials, $51.4+/-6.8 \mathrm{~cm}$ without arms in the NS trials, $70.8+/-11.7 \mathrm{~cm}$ with arms in the SS trials and $76.3+/-14.5$ $\mathrm{cm}$ without arms in the SS trials. In the medial-lateral plane, the COM moved on average to the right $2.38+/-1.6 \mathrm{~cm}$ with arms in the NS trials, $2.56+/-1.6 \mathrm{~cm}$ without arms in the NS trials, $5.55+/-2.1 \mathrm{~cm}$ with arms in the SS trials and $6.26+/-3.3 \mathrm{~cm}$ without arms in the SS trials. Vertically, the COM lowered on average $0.60+/-0.34 \mathrm{~cm}$ with arms in the NS trials, $0.68+/-0.35 \mathrm{~cm}$ without arms in the NS trials, $3.17+/-1.9 \mathrm{~cm}$ with arms in the SS trials and $5.61+/-4.2 \mathrm{~cm}$ without arms in the SS trials. An increased travel distance demonstrates that the subjects were unable to stop forward progression on the slippery surface as effectively as stopping on the normal surface; a result supporting the increased deviation of the COPX. The large medial-lateral movement parallels the COP data which also showed greater mediallateral movements during SS trials compared to the NS trials. Subjects significantly lowered their COM during the SS trials in an attempt to regain stability on the slippery surface.

COM momentum (Figure 2.5A) was significantly different during the SS trials when compared to peak vertical NS trial values (Figure 2.5B). Both horizontal velocities were significantly greater in the SS trials than the NS trials; subjects were sliding forward and their COM was traveling to the right faster than during NS trials when slipping on the rollers. The vertical COM momentum was increasing in the NS trials and decreasing in the SS trials. The lowering of the COM is likely a response to the slip to stabilize the body and maintain balance. The increase in the COMZ momentum could be the result of a momentum transfer from A-P to M-L to dissipate forward momentum. 
Arm movement did not have a significant effect on COM displacement. Arm movement did, however, have a significant effect on COM momentum. When peak COM momentum values were compared between SS trials using a full or arm-less COM model, there was

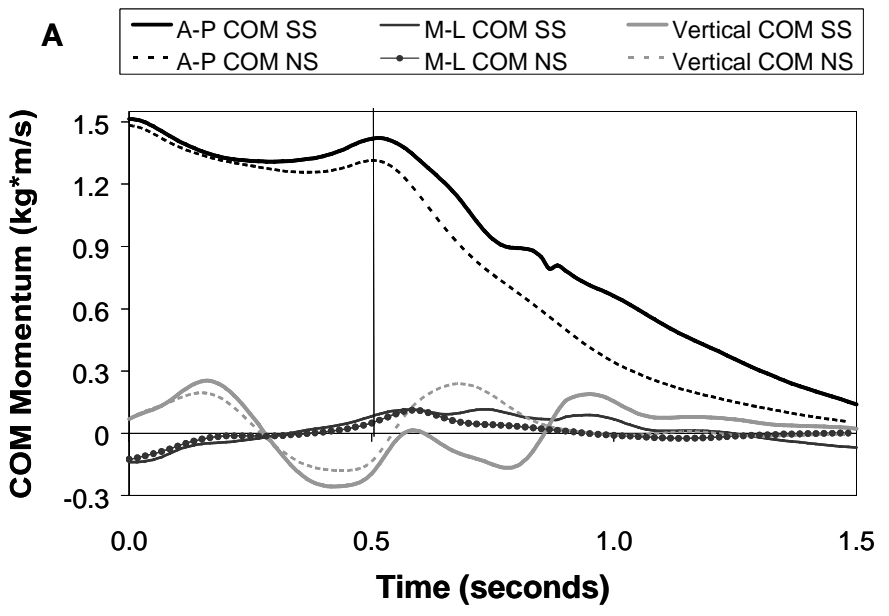

B
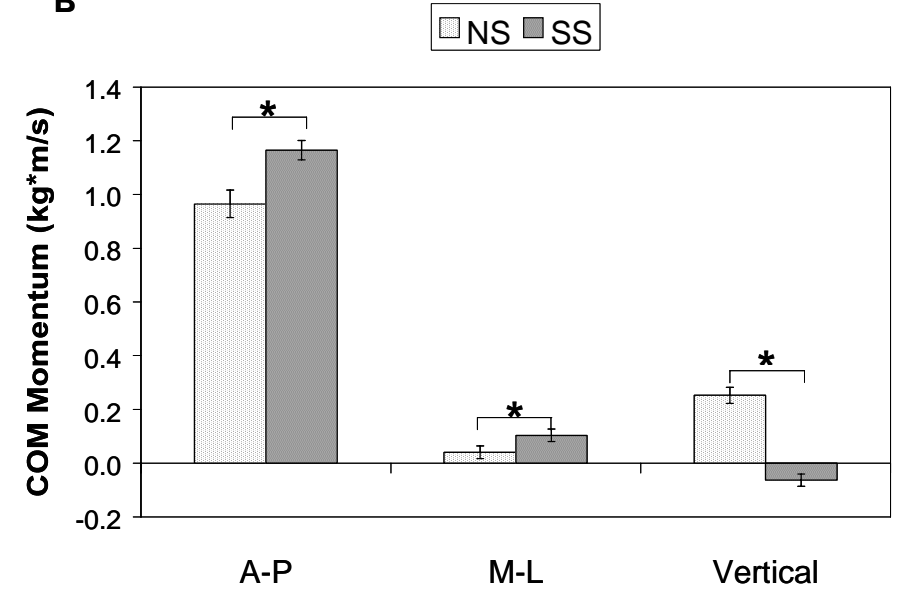

Figure 2.5: A) Average COM momentum profiles. Vertical line indicates heel contact on the rollers. Solid lines represent SS trials, dashed lines represent NS trials. B) Comparison at peak vertical COM momentum during NS trials with SE. * = significant difference between conditions $(\alpha<0.05)$. $n=6$ subjects for $A$ and $B$.

significantly higher forward momentum without the arms in the model (Figure 2.6). The increased forward momentum suggests that the arms were effectively used to arrest forward 
momentum during the slip and, as the arms were neither fully flexed nor abducted, the arms could have possibly assisted with a transfer of momentum from forward to lateral.

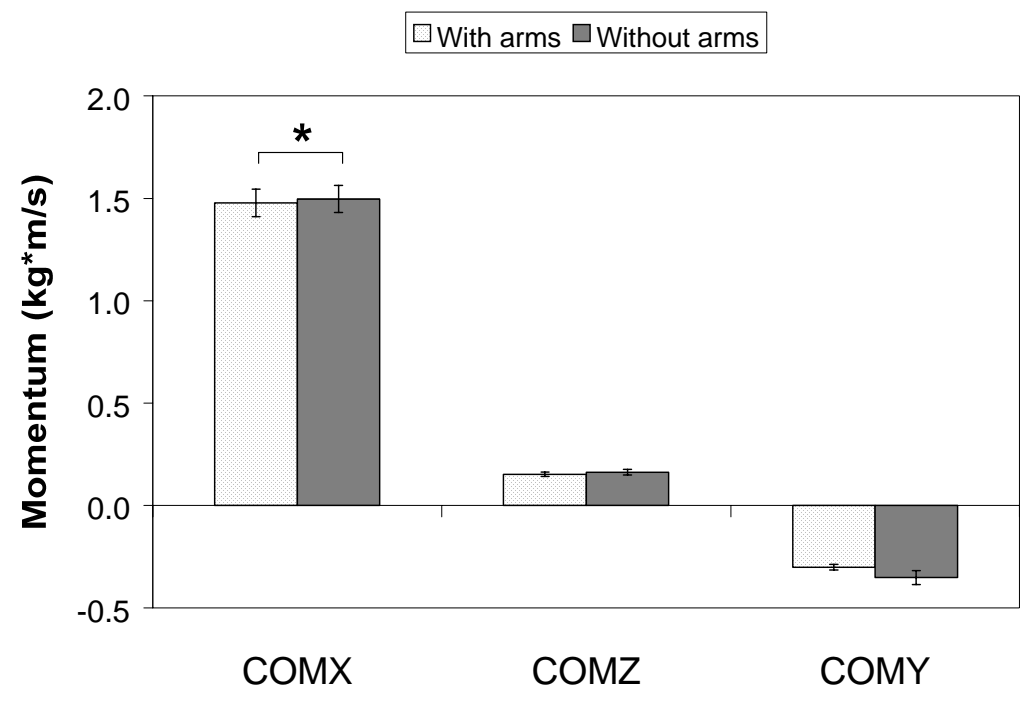

Figure 2.6: Average peak COM momentum values compared between a full and an arm-less COM model with SE during SS trials. COMX and COMZ values were compared at maximum values, COMY values were compared at the minimum value. $*=$ significant difference between conditions. $(\alpha<0.05) . \mathrm{n}=6$ subjects.

\subsubsection{Muscle Activity}

\subsubsection{Non-slippery stops versus walk-throughs}

On average, all muscles, except for the right $\mathrm{TA}$ and right $\mathrm{RF}$, showed significantly different activity during the NS compared to the WT trials (Figure 2.7 for the right limb and Figure 2.8 for the left limb). Average latencies between WT and NS trials ranged from $\sim 57 \mathrm{~ms}$ (right LES) to $332 \mathrm{~ms}$ (right BF) (Table 2.1). Inhibition was seen in the main propulsion muscle of the left limb (SOL) along with the left LES. The left hip and knee muscles (RF and BF) co-contracted to slow the swing of the left limb during gait termination. 
The left SOL was inhibited to prevent acceleration during the toe-off of the left limb. The left TA increased its activation to dorsiflex the ankle which provided sufficient toe clearance during swing. The LES increased activation bilaterally to stop forward rotation of the trunk. The right RF and SOL seemed to generate most of the braking force for the right limb while the right TA and BF slightly increased activation levels, probably to support the ankle and hip joints, respectively, through co-contraction.

Table 2.1: Difference latencies comparing NS to WT and SS to NS trials. Values are means \pm SE. $\mathbf{n}=$ number of subjects who displayed significantly different muscle activation in $\geq 60 \%$ of NS trials compared to WT trials and the number of subjects who displayed significantly different muscle activation in slippery stop trial (SS) compared to NS trials. ${ }^{\wedge}=$ difference was inhibitory. Units are in ms.

\begin{tabular}{lcccc}
\hline Muscle & NS vs. WT & $\mathbf{n}$ & SS vs. NS & $\mathbf{n}$ \\
\hline RTA & $236 \pm 53$ & 4 & $94 \pm 19$ & 8 \\
RSOL & $162 \pm 91$ & 4 & $56 \pm 24$ & 8 \\
RRF & $150 \pm 46$ & 3 & $91 \pm 14$ & 8 \\
RBF & $332 \pm 48$ & 8 & $66 \pm 19$ & 8 \\
RLES & $57 \pm 17$ & 7 & $105 \pm 38$ & 8 \\
LTA & $136 \pm 39$ & 8 & $124 \pm 56$ & 8 \\
LSOL & $199^{\wedge} \pm 44$ & 7 & $178 \pm 30$ & 7 \\
RF & $155 \pm 31$ & 8 & $122 \pm 16$ & 8 \\
LBF & $136 \pm 38$ & 5 & $36 \pm 8$ & 8 \\
LLES & $115^{\wedge} \pm 51$ & 5 & $41 \pm 17$ & 8
\end{tabular}

2.3.5.2 Slippery surface stops versus Non-slippery surface stops.

All muscles showed significantly increased activity during the SS trials when compared to the NS trials (Figures 2.7 and 2.8). The difference latencies ranged from $\sim 36 \mathrm{~ms}$ (left BF) to 178ms (left SOL) (Table 2.1). As the right foot slid forward, the right TA increased its activity in an attempt to bring the shank over the foot and, therefore, the COM 
over the base of support. The increased right SOL activity stiffened the ankle joint and increased ankle stability. Both the right RF and BF increased activity to prevent collapse and lower the COM, respectively. The co-contraction of both thigh muscles would also increase stability at the knee and hip and provide support to the upper body as the COM lowers. The bilateral increase in LES activity is likely an attempt to stop forward trunk progression. 
WT trials
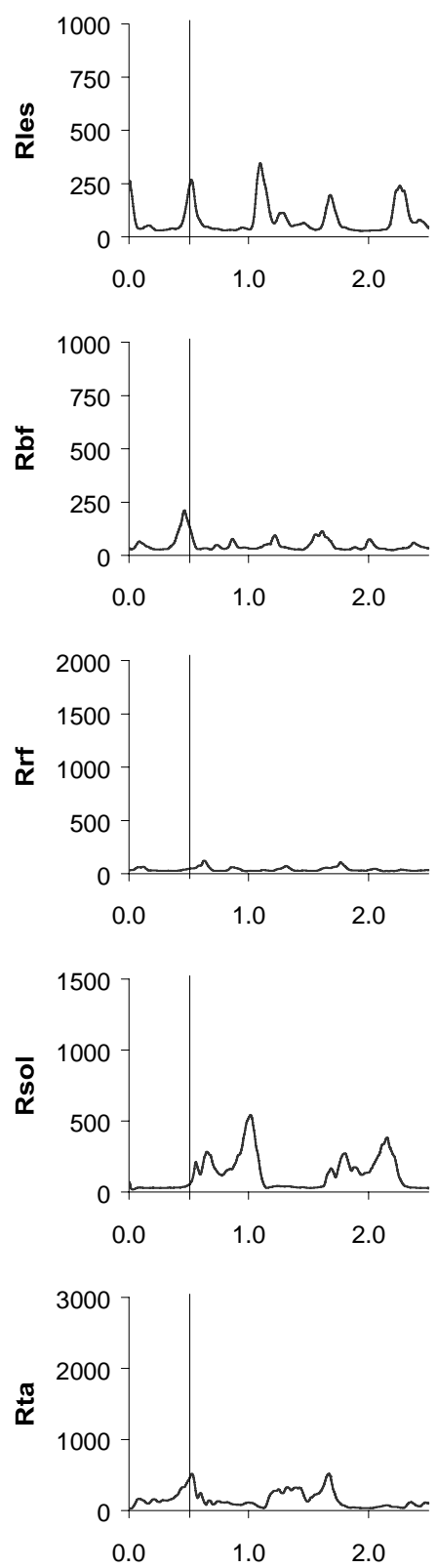

NS trials
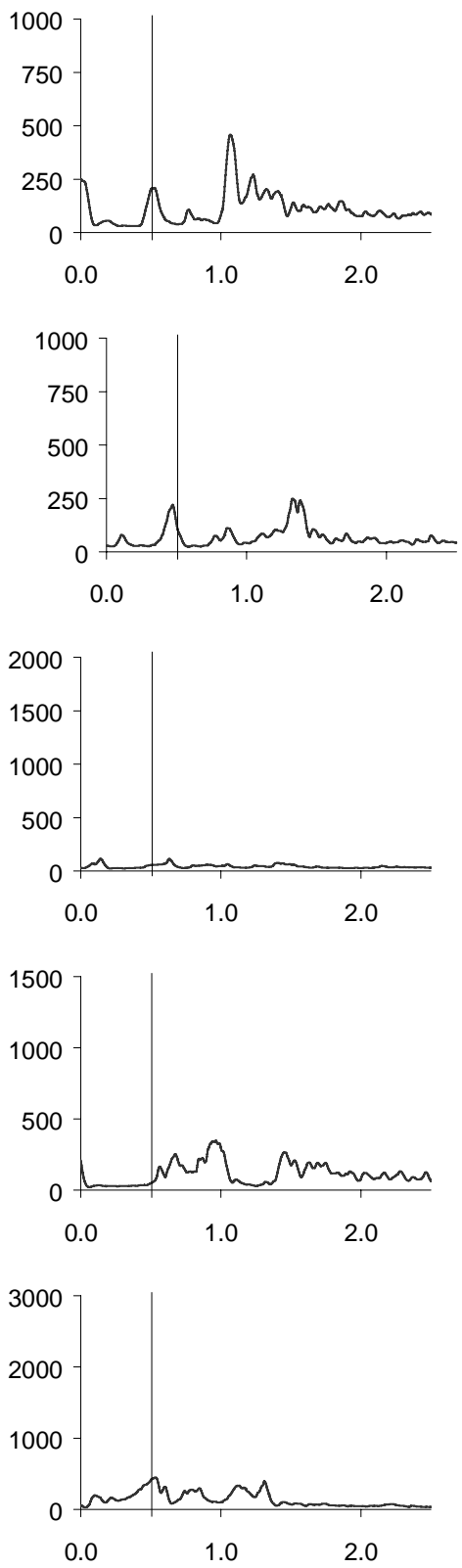

$\underline{\mathrm{SS} \text { trial }}$
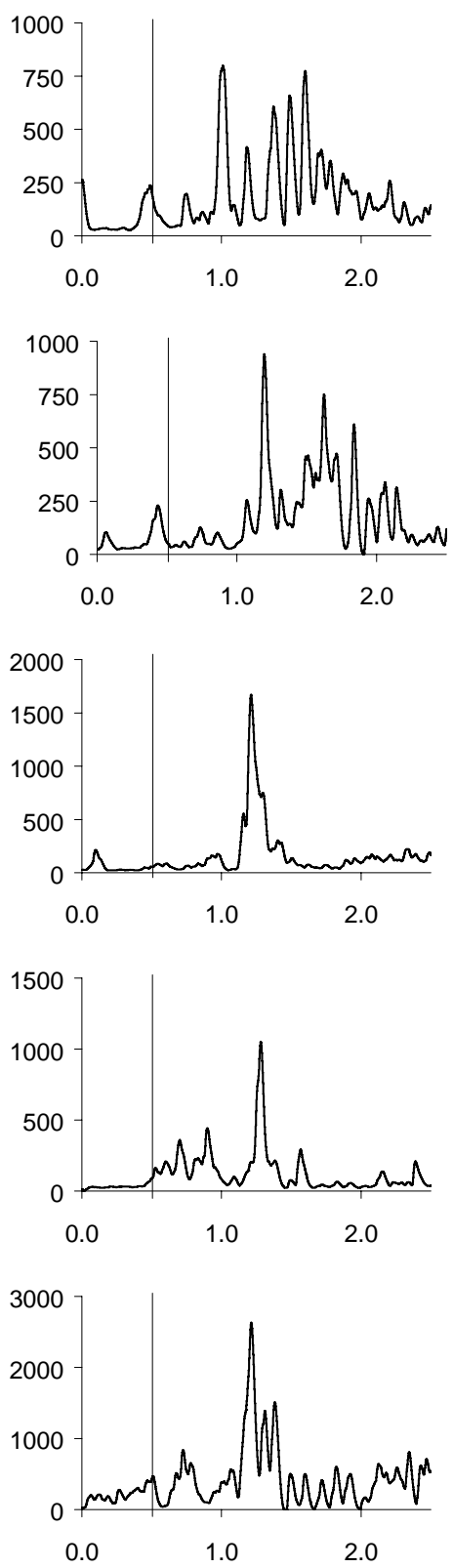

Figure 2.7: Representative right limb EMG from one subject. Vertical line represents time of heel contact on the rollers. Horizontal axis is time (s). Signal to stop at 0 s. Vertical axis is arbitrary units. 

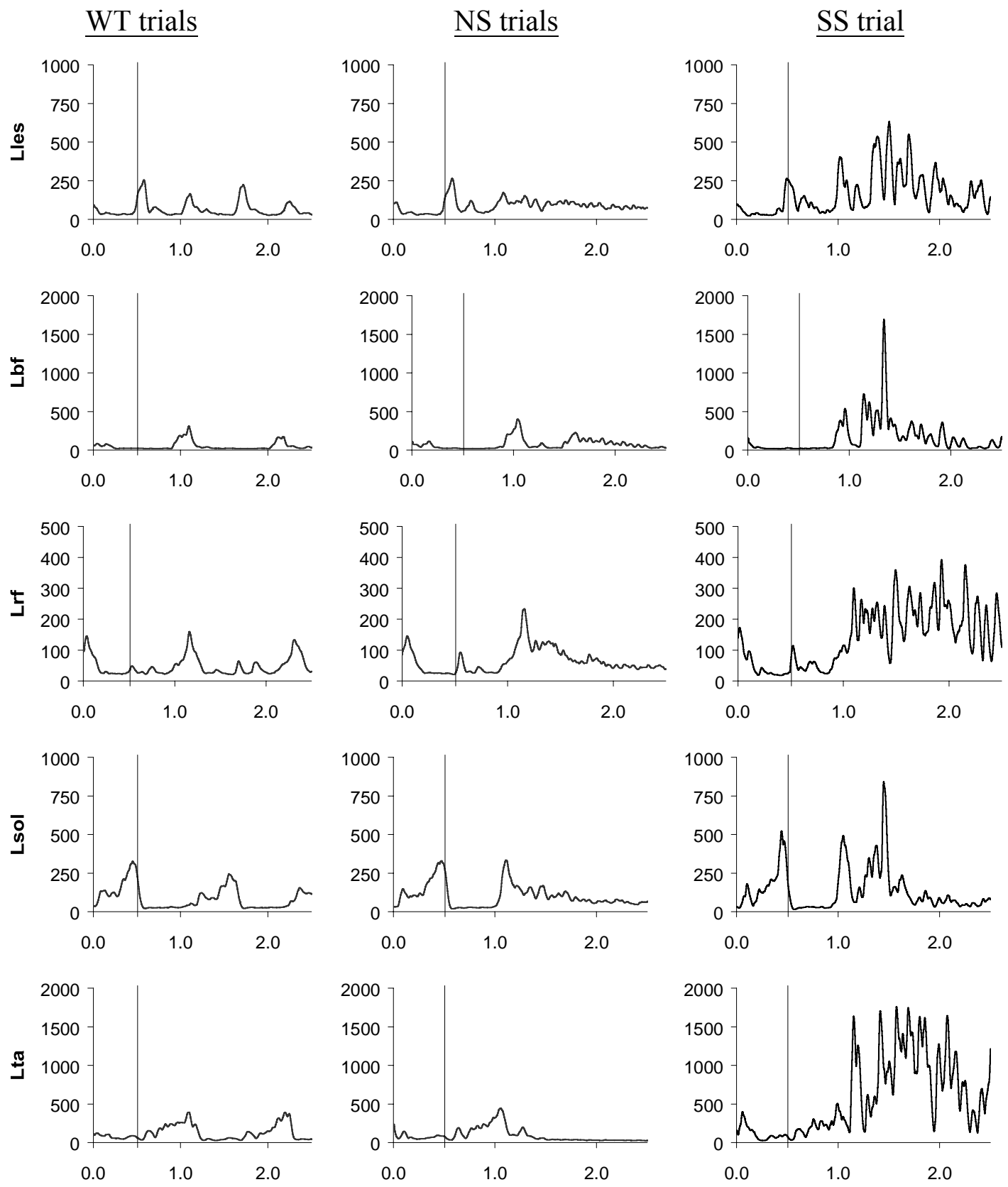

Figure 2.8: Representative left limb EMG from one subject. Vertical line represents time of heel contact on the rollers. Horizontal axis is time (s). Signal to stop at 0s. Vertical axis is arbitrary units. 


\subsection{DISCUSSION}

In this study we show that subjects unload their left limb to decrease acceleration, load their right limb to increase braking force and manipulate the COP movement underneath the feet to stop successfully. When attempting to stop on a slippery surface each subject displayed a slightly different reaction yet a generalized response to the slippery surface emerged with included an arm raise, a shortened final step, and increased lower limb muscle activity to support the lowering COM; all strategies were employed to regain stability and prevent a fall. The reaction to the slippery surface in the present study is similar to previously reported perturbation reactions during locomotion (e.g. Marigold and Patla 2002; Misiaszek 2003) suggesting a generalized recovery strategy to perturbations during walking that attempts to maintain balance and the specific locomotor task.

\subsubsection{Walk-throughs versus gait termination}

GT differed from normal walk-through trials primarily by decreased forward propulsion and increased deceleration of the body after the stop signal. There was no pushoff phase after the right foot was placed on the locked rollers through the ground reaction forces. Co-contraction at the hip and knee slowed the left leg during the final swing phase, while inhibition of the left SOL decreased the push-off forces. The right limb increased its braking force through an extensor synergy (increased RF and SOL activity) while the remaining muscles in the right limb (TA and $\mathrm{BF}$ ) provided support through an increase in stiffness and, therefore, stability. Trunk movement was controlled through bilateral LES activation. Although muscle activity from large groups such as the gluteals and vasti group 
were not recorded, their activity likely coincided with the BF and RF muscles respectively to assist with the extensor synergy and decelerate forward movement.

Compared to previously reported results (Crenna et al. 2001) the onset of muscle activity signaling the initiation of gait termination were much quicker for the right limb [average latency $\sim 180 \mathrm{~ms}$ for the right limb compared to $\sim 330 \mathrm{~ms}$ for the swing limb in Crenna et al. (2001)], whereas the left/stance limb latencies match very closely [150ms for the left limb and $\sim 150 \mathrm{~ms}$ for the stance limb (Crenna et al. 2001)]. There was no clear recruitment order evident in our study whereas Crenna et al. (2001) found a distinct distal-toproximal activation in the stance limb (our left limb) and a proximal-to-distal activation in the swing limb (our right limb). These differences in recruitment order may be related to the experimental protocol. The present study used a $25 \%$ chance of gait termination while the comparable study (Crenna et al. 2001) employed a 50\% occurrence of gait termination. Higher probability of GT in the study by Crenna et al. (2001) would reduce the variability of the program for GT. Perhaps the fewer termination trials in the current study allowed for larger variance thereby making it difficult to discern any clear ordering of muscle activation patterns. Another source of variance between the two studies could be the selection of trials for analysis: The present study included all successful NS trials (i.e., the subject stopped with both feet on the rollers) whereas it is unclear if all of the stop trials were included for analysis in Crenna et al. (2001).

Flexor and extensor synergies (Hase and Stein 1998) were used to load and unload the limbs for effective gait termination as shown in the kinetic data from the force plates. 
That is, the flexor muscle activity increase in the left limb decreased the push-off force under the left limb. The braking forces under the right limb increased as a result of increased extensor activity. Through the flexor/extensor synergies, subjects used the ground reaction forces to manipulate the COP and decelerate the COM to a stable, stationary position.

The muscle activation patterns in the present study are comparable to those of Hase \& Stein (1998) who reported an inhibition in the left limb (comparatively) SOL and a similar latency of left TA activity [150-200ms reported in Hase and Stein (1998), approx 140ms in present study]. Differences exist when comparing the latencies of the left and right LES muscles; the present study found latencies $\sim 115 \mathrm{~ms}$ and $57 \mathrm{~ms}$, respectively, where Hase and Stein (1998) reported a bilateral activation of the LES muscles $\sim 200 \mathrm{~ms}$. In general, the present study found latencies from $\sim 15-150 \mathrm{~ms}$ shorter than those reported in Hase and Stein (1998). The differences may be due to the stimulus provided to initiate gait termination. Hase and Stein (1998) used electrical stimulation of the superficial peroneal (SP) nerve whereas the present study used a visual cue. Electrical stimulation of the SP nerve may have slightly prolonged activation based on the amount of neural processing involved to perceive the signal and initiate GT and also the experience interpreting the cue. A visual cue requires perception at the cortical level followed by appropriate cortical-spinal commands to stop walking. Stimulating the SP nerve requires perception of the cue, interpretation of the cue as a signal to stop walking and then initiation of a GT program: A route that involves a signal traveling up the spinal cord to the cortex, cortical processing and subsequent cortical-spinal commands to stop walking. The sight of a stop sign is a regular occurrence in everyday life 
and is easily interpreted as a cue to stop. SP nerve stimulation, however, is not regularly used as a cue to stop movement and may have required more processing to understand the significance of the cue.

\subsubsection{Gait termination on a normal surface versus a slippery surface}

Gait termination on the slippery surface of the unlocked rollers proved to be a balance-challenging task. Normal stopping strategies were insufficient to maintain COM trajectories while slipping. Subjects were unable to generate enough braking force to stop the forward progression of the COM within the same limits as normal gait termination. In all SS trials, subjects shortened their last step with their left leg (i.e., the step that would place the left foot beside the right foot on the rollers). This small step enabled the subjects to increase their base of support during the SS trials which would allow the ground reaction forces (i.e., the COP) under the left foot to corral the COM and help prevent a fall. Subjects also raised their arms and lowered their COM to stabilize their body and stop walking.

Every muscle recorded increased its activity to support the body, stop forward movement and restore stability. All of the lower limb muscle activity increased to support the legs and the whole-body COM and, most likely, to stiffen the lower limb joints throughout balance recovery. The difference latencies between the NS and SS trials suggest that the nervous system is able to detect the slip and elicit a behavioural response in as little as 60ms; these latencies suggest long-latency reflexes $(57 \mathrm{~ms})$ as well as voluntary reactions $(178 \mathrm{~ms})$ to the slippery surface (Pearson and Gordon, 2000). The large left TA activity may have created a toe lift to prepare for the shortened step. The shortened step and increased TA activity 
resembles the startle response observed by Nieuwenhuijzen et al. (2000). The startle response is an adaptive response in that the nervous system attempts to adopt the most stable posture in an unstable situation. The arm raise and increased TA activity helped to stabilize the falling COM during the slip. While all subjects were surprised by the slippery surface, it is likely that any apparent startle response was initiated as part of a balance recovery program to reinstate stability but was not the primary method by which subjects successfully terminated gait.

Medial-lateral plane movement was increased during SS trials as evidenced in the larger COP and COM deviations while slipping. This increase in movement cannot be attributed to the rollers since they permit slipping only in the direction of progression. A counter-clockwise twist of the body, caused by the right foot sliding forward while the left foot was behind the rollers, may have initiated the medial-lateral movement by turning the body axis from its original alignment. A twist may also have been a reaction to and not a consequence of the right foot sliding forward. If the basic GT strategy (involving loading and unloading the limbs and COP manipulation) is not effective, the CNS may have attempted to transfer part of forward momentum to lateral momentum; a transfer which would involve some rotation about the vertical axis.

To stop successfully on the rollers subjects may have attempted to transfer forward to lateral momentum, as seen in previous research on patients with balance deficits (O'Kane et al. 2003). Previous GT investigations in both healthy (Hase and Stein 1998) and neurologically impaired individuals (O’Kane et al. 2003) observed energy/momentum 
transfers during gait termination. Hase and Stein (1998) suggested that subjects used a toeraise to dissipate forward momentum if they were unable to either effectively dissipate pushoff force or generate sufficient braking force. By transferring momentum between axes, subjects in the present study may have utilized a lateral limb load/unload strategy instead of an anterior-posterior load/unload strategy found in normal stopping (Hase and Stein 1998; B McGowan unpublished observations). O'Kane et al. (2003) reported that both cerebellar and vestibular patients used a forward to lateral energy transfer to assist GT. The cerebellar patients were unable to control the eccentric muscle activity required to absorb the forward momentum while the vestibular patients were not able to detect the amount of acceleration of the body until the last step when the visual and proprioceptive systems provided information about the velocity of movement. The awareness of a lack of deceleration in the vestibular patients during this last step would eliminate the usefulness of a reduced push-off power (i.e., it would be too late at that point in GT) and therefore require a large braking force and a momentum transfer to effectively dissipate forward momentum. These findings applied to the current study suggest that, when traditional stopping strategies such as decreased push-off and increased braking forces fail to stop forward progression, subjects attempt to transfer forward momentum to lateral momentum to effect safe, stable gait termination.

Lowering the COM increases stability (Tucker et al. 1998; Marigold and Patla 2002; Cham and Redfern 2001) and was a strategy used by all subjects during SS trials. The lowering of the COM was likely the result of the flexor synergy often seen in slips (Brady et al. 2000; Cham and Redfern 2001, 2002; Marigold and Patla 2002), evidenced by the 
increased muscle activity in the hip, knee, and ankle flexors of both limbs. The slip of the right foot on the rollers may have passively contributed to the lowering of the COM. Since in some subjects, the COM began moving upwards before the foot had stopped sliding forward, the observed COM lowering cannot be considered a passive consequence of the foot sliding on the rollers. This suggests that lowering of the body COM is most probably an active control strategy.

Arm movement was ineffective on average in altering the COM displacement during SS trials. The arms did, however, make a significant difference in the peak forward momentum values suggesting the arms helped dissipate forward momentum. The arm strategy, therefore, is used to control movement velocity instead of displacement. This protective arm elevation strategy is often seen in slips and is coordinated with the lower limb slip response (Haridas and Zehr 2003; Marigold and Patla 2001; Marigold et al. 2003; Misiasek 2003). The interlimb coordination serves to diminish the whole-body COM momentum during the slip, minimize the perturbation and assist in balance recovery.

\subsubsection{Neural mechanisms}

Normal GT was successfully performed in the majority of trials. The instructions given to the subject about the requirement to stop when the visual cue was given allowed subjects to plan a GT strategy. The timing of the cue required the subject to stop within one step. During this final step, the subject would have had to process the visual cue as a stop signal and initiate a GT program involving an extensor/flexor or load/unload synergy in the limbs. The number of catch (WT) trials and the timing restraint on the response removes the 
chance for any anticipatory actions. The change in motor activity from a locomotor to a GT program was initiated as early as $57 \mathrm{~ms}$ and took as long as $332 \mathrm{~ms}$ on average. The GT program was likely initiated by the visual cue and monitored by the visual, proprioceptive and vestibular sensory systems.

The nervous system initiated slip responses in some muscles in as little as $36 \mathrm{~ms}$. These initial reactions are suggestive of stretch reflex, or short-latency reactions (Pearson and Gordon 2000). On average, most motor activity recorded was between 50 and 200ms suggestive of polysynaptic or long-latency reflexes (Pearson and Gordon 2000) and simple reaction times (Hase and Stein 1998). It is possible that the vestibular system detected the slip through head acceleration (Horak et al. 1994) and initiated extensor activity to support the body during the perturbation. Cutaneous receptors on the plantar surface of the foot (Perry et al. 2000) and load-sensitive afferents in the ankle extensors (Misiaszek et al. 2000) likely detected the slip, and through their afferent feedback, helped initiate a polysynaptic response. It is unlikely that the visual system was able to detect the slip and, in sufficient time, elicit a visually-based balance-correcting response due to the relative slowness of normal visual reaction time $(\sim 100 \mathrm{~ms}$ delay compared to proprioceptive-based balance correcting responses (Pearson and Gordon 2000)). Comparison of the slip reaction during GT seen here to previous studies involving perturbations to either the support surface (Cham and Redfern 2001, 2002: Marigold and Patla 2002: Marigold et al. 2003) or the loading of the lower limbs during steady state gait (Misiaszek et al. 2000; Misiaszek 2003) suggests that the CNS generates a common balance-recovery strategy when equilibrium is disturbed during 
locomotor activities. The reaction latencies could be interpreted as responses from spinal and supraspinal structures (such as the lateral vestibular nucleus, reticular formation, and motor cortex) charged with monitoring locomotor activity and maintaining dynamic balance throughout.

In conclusion, muscle responses to slipping are comparable to responses found in previous experiments (Cham and Redfern 2001, 2002; Marigold et al. 2003; Marigold and Patla 2001) as are the arm responses (Marigold et al. 2003) and shortened step (Cham and Redfern 2002; Marigold et al. 2003) suggesting a general balance recovery strategy when a slippery surface is encountered during GT. This recovery strategy includes an overall increase in muscle activity to increase lower extremity joint stiffness, lowering of the COM, a shortened step, and arm elevation; all designed to increase stability and maintain balance during the slip. Gait termination on a slippery surface employs the same recovery strategy but includes an arm raise to stop forward progression and transfer forward to lateral momentum to stop safely and prevent a fall.

\subsection{ACKNOWLEDGEMENTS}

Special thanks to M. Ishac for software support. This research was supported by NSERC and CIHR. 


\subsection{REFERENCES}

Brady RA, Pavol MJ, Owings TM and Grabiner MD. Foot displacement but not velocity predicts the outcome of a slip induced in young subjects while walking. J. Biomech, 33: 803-808, 2000.

Cham R, and Redfern MS. Lower extremity corrective reactions to slip events. J. Biomech, 34: 1439-1445, 2001.

Cham R, and Redfern MS. Changes in gait when anticipating slippery floors. Gait Posture, 15: 159-171, 2002.

Crenna P, Cuong DM, and Brénière Y. Motor programmes for the termination of gait in humans: organization and velocity-dependent adaptations. J. Physiol, 537.3: 1059-1072, 2001.

Haridas C, and Zehr EP. Coordinated interlimb compensatory responses to electrical stimulation of cutaneous nerves in the hand and foot during walking. J Neurophysiol, 90: 2850-2861, 2003.

Hase K, and Stein RB. Analysis of rapid stopping during human walking. J Neurophysiol, 80: 255-261, 1998.

Horak FB, Shupert CL, Dietz V, and Horstmann G. Vestibular and somatosensory contributions to responses to head and body displacements in stance. Exp Brain Res, 100: 93-106, 1994.

Jaeger R, and Vanitchatchavan P. Ground reaction forces during termination of human gait. J Biomech, 25 (10): 1233-1236, 1992.

Jian Y, Winter DA, Ishac MG, and Gilchrist L. Trajectory of the body COG and COP during initiation and termination of gait. Gait Posture, 1: 9-22, 1993.

Marigold DS, and Patla AE. Strategies for dynamic stability during locomotion on a slippery surface: effects of prior experience with and knowledge about surface characteristics. J Neurophysiol, 88: 339-353, 2002.

Marigold DS, Bethune AJ, and Patla AE. Role of the unperturbed limb and arms in the reactive recovery response to an unexpected slip during locomotion. J Neurophysiol, 89: 1727-1737, 2003.

Misiaszek JE. Early activation of arm and leg muscles following pulls to the waist during walking. Exp Brain Res, 151: 318-329, 2003.

Nieuwenhuijzen PHJA, Schillings AM, Van Galen GP, and Duysens J. Modulation of the startle response during human gait. J Neurophysiol, 84: 65-74, 2000.

O'Kane FW, McGibbon CA, and Krebs DE. Kinetic analysis of planned gait termination in healthy subjects and patients with balance disorders. Gait Posture, 17: 170-179, 2003.

Pearson K, and Gordon J. Spinal Reflexes. In: Principals of Neural Science, edited by Kandel ER, Schwartz JH, and Jessel TM. Toronto, ON, McGraw-Hill Health Professions Division: 2000, pp. 713-736.

Perry SD, McIllroy WE, and Maki BE. The role of plantar cutaneous mechanoreceptors in the control of compensatory stepping reactions evoked by unpredictable, multi-directional perturbation. Brain Res, 877: 401-406, 2000. 
CHAPTER 3: ADAPTATION TO GAIT TERMINATION ON A SLIPPERY SURFACE IN YOUNG, HEALTHY ADULTS 


\subsection{INTRODUCTION}

This study examines the changes in gait termination with knowledge of and experience with a slippery surface. Previous research investigating unexpected perturbations during gait, including a slip perturbation, describes a typical lowering of the body's centre of mass (COM) (Oates et al 2005; Marigold and Patla 2002), an arm raise (Oates et al 2005; Misiaszek 2003), and a shortened subsequent step (Marigold et al 2003). The muscle activity in the lower limbs increase to prevent collapse (Ferber et al 2002; Cham and Redfern 2000; Misiaszek et al 2000) and persist with the planned motor program (Marigold et al 2003; Tang et al 1998b) whether it is continued gait or gait termination (Oates et al 2005).

Prior experience with a slippery surface causes changes in various postural and gait characteristics to diminish the perturbing effect of the slip. Adaptations include shorter steps (Brady et al 2000; Cham and Redfern 2002; Heiden et al 2006; Myung and Smith 1997), diminished arm responses (Marigold and Patla 2002), increased margin of stability between the COM and the base of support (Marigold and Patla 2002) and a decreased foot-floor angle to decrease the shear forces required to prevent a slip (Brady et al 2000; Cham and Redfern 2002; Marigold et al 2003; Marigold and Patla 2002),. The magnitude of the muscular response diminishes quickly, often in one trial, as the response to the slip becomes more efficient (Henry et al 1998; Marigold et al 2003; Marigold and Patla 2002; Tang et al 1998b). In these previous studies, both prior experience and knowledge could account for the observed adaptive behaviours. 
There can only be one truly unexpected slip. After being alerted to the possibility of a slip, behaviour changes in preparation for the surface (Brady et al 2000; Cham and Redfern 2002; Heiden et al 2006; Marigold and Patla 2002; Myung and Smith 1997). We wanted to control behaviour in order to examine the adaptive changes in response to the slippery surface and not in expectation of a slippery surface. We therefore provided knowledge of when a slippery surface occurred so the adaptive behaviours would be present only during the slippery stops and not during the non-slippery stops. The purpose of this study is to examine the organization of the adaptation to a slippery surface during gait termination in young healthy adults. We hypothesize that adaptations to the slippery surface will occur within the first two trials after an unexpected slip and that behaviour will change from a supportive (a reactive strategy to prevent collapse during the stop) to a surfing (a proactive strategy to maintain stability during the stop) strategy.

\subsection{MATERIALS AND METHODS}

\subsubsection{Protocol}

The adaptation to stopping on a slippery surface was investigated in eight young, healthy participants (four males and four females, mean age $+/-\mathrm{SD}=24,+/-3$ years). Experimental set-up included a four-inch high wooden walkway with aluminum rollers covering one force plate (See Marigold and Patla, (2002) for detailed description of the roller apparatus).As the subject stepped with their left foot on the first force plate (covered by wood), a monitor at the end of the walkway signaled for the subject to stop with both feet on 
the second force plate (covered by the rollers) (figure 3.1 shows the set-up of the laboratory during testing). After a series of random stop trials within walk-through trials, the rollers were unlocked without the knowledge of the subject to provide a slippery surface. The subject was then signaled to stop during this trial to produce a purely unexpected slip trial (unexpected slippery stop trial). The rollers were aligned so the slip was only in the direction of progression and participants slipped on and over the top of the rollers. (Data reporting comparisons between non-slippery stops and the first slippery stop have been reported previously; Oates et al 2005).

After the first slip trial the subject was informed that the display would randomly ask the person to stop on the rollers. In five of the stop trials the rollers were unlocked to create a slippery surface and the display at the end of the walkway read "Stop Slippery". These trials were called the cued slippery stop trials. The other five stop trials were non-slippery stops and the display at the end of the walkway read "Stop". These trials were called the cued nonslippery stop trials. These ten stop trials (five slippery, five non-slippery) were randomly placed within fifty walk-through trials to discourage anticipation of the stop trials. The protocol was approved by the University of Waterloo Ethics committee and safety measures were taken to ensure participants did not fall during any of the trials.

\subsubsection{Kinetics}

Two $_{\text {AMTI }}{ }^{\odot}$ force plates were used to collect ground reaction forces sampled at 2400 Hz. Previous testing (A Oates unpublished observations) of the force plates with the wooden platform and roller coverings demonstrated accurate (within 2\%) data recording, with the 
exception of the natural frequency and the sensitivity of the system. These system differences were deemed acceptable for this experiment. Force plate data were low-pass filtered at $60 \mathrm{~Hz}$ and analyzed using a custom program (MIshac Kinetics).

Braking impulse was calculated as the integration of the horizontal ground reaction force in the direction of progression over a set time period. The integration period was determined by the length of foot contact on the rollers during the unexpected slippery stop trials. This integration period was constant within a subject but varied between subjects according to the length of foot contact by each subject on the slippery surface (range $=0.12$ to 0.35 seconds). The peak propulsive force and the propulsive impulse (integrated propulsive force) were calculated for the entire time propulsion was created by the trail/left limb and compared between conditions. The braking and propulsive impulses and the peak braking and propulsive forces were compared between unexpected slippery stop, cued slippery stop and the average of the cued non-slippery stop trials conditions.

\subsubsection{Kinematics}

Three Optotrak@ (Northern Digital Inc., Waterloo) cameras recorded kinematic data from 21-IREDs (infra-red emitting diodes) at $60 \mathrm{~Hz}$ to create a 12 -segment model for centre of mass (COM) calculation. IREDs were placed at: xyphoid process and bilaterally at the fifth metatarsal head, heel, lateral malleolus, lateral femoral condyle, greater trochanter, ASIS, acromion process, olecranon, radial styloid, and medial clavicle. Raw data was processed using Optofix and CofM software (MIshac Kinetics) and low-pass filtered at $6 \mathrm{~Hz}$. For two of the participants, the marker data on the elbows were inadequate to provide 


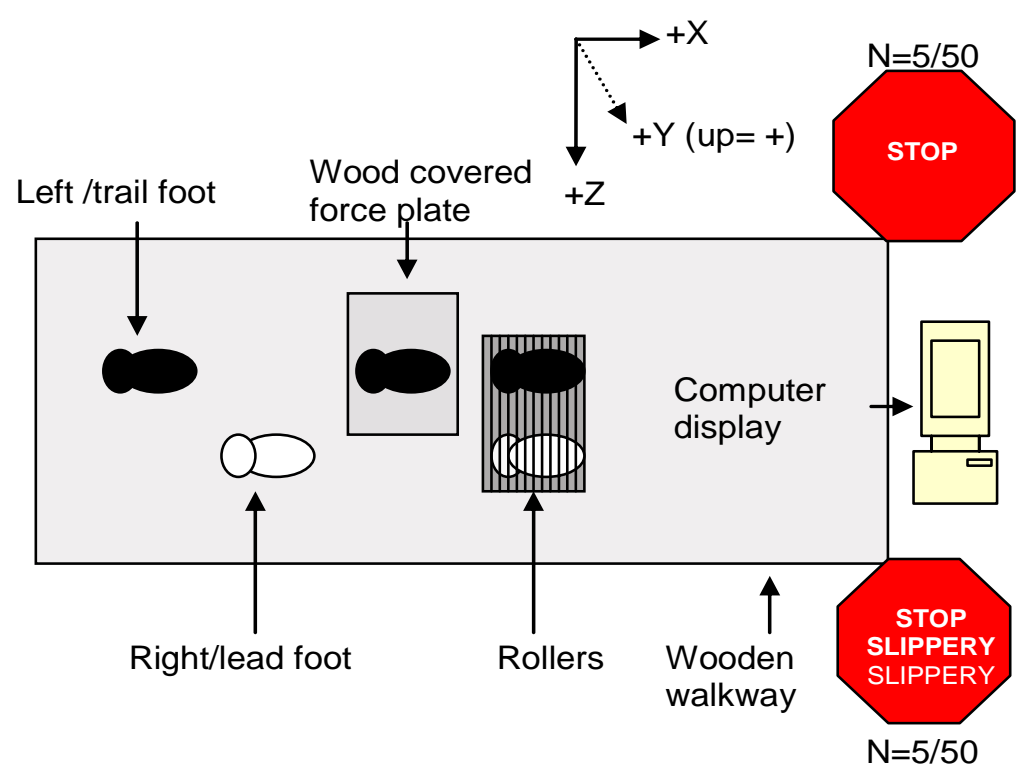

Figure 3.1: Diagram of laboratory set-up and display screens. Diagram is not to scale.

sufficient time periods over which to observe the COM movement; therefore, the arms were removed from the COM model to create a 10-segment COM model.

Peak downward displacement of the COM was calculated from the time of heel contact on the rollers for 500ms. Values of the peak downward COM position were normalized to the average value during walk-through trials to account for different heights of the participants. The anterior-posterior (A-P) positions of the COM and the heel marker of the lead limb were compared to show the difference at heel contact on the rollers during gait termination. The angle of the foot at heel contact on the rollers was calculated using the heel and $5^{\text {th }}$ metatarsal markers $\left(\theta=\tan ^{-1}(\right.$ vertical metatarsal position - vertical heel position/ A-P metatarsal position - A-P heel position)). Qualitative assessment of the video data showed that the arm raise was produced with a fairly straight arm and, therefore, the maximum height of both arms was calculated as the maximum height of the marker on the radial styloid within the first 500ms after heel contact on the rollers. Values were normalized to the slippery stop trial to account for different arm lengths and body heights. 
The forward velocity of the COM at heel contact of the trail limb on the woodcovered force plate was measured to demonstrate any changes in the forward velocity approaching the rollers. Time to slow was defined as the time when the COM forward velocity fell below the average minus two standard deviations of the average forward velocity of the walk-through trials. The time to stop was defined as the time when forward velocity fell within the average plus two standard deviations of the average velocity (over $500 \mathrm{~ms}$ ) one second after the right heel contact on the rollers of the cued non-slippery stop trials (as reported in Oates et al 2005).

\subsubsection{EMG}

Muscle activity was recorded from 10 pairs of bipolar Kendall Meditrace ${ }^{\circledR}$ (Chicopee, MA) surface electrodes: tibialis anterior (TIB), soleus (SOL), rectus femoris (RF), biceps femoris (BF), and lower erector spinae (LES), bilaterally) sampled at $2400 \mathrm{~Hz}(0.5-1 \mathrm{~K}$ gain with a Bortec ${ }^{\odot}$ AMT-8 amplifier). EMG data were analyzed using a custom-designed Matlab program (Mathworks, Natick, MA) which full-wave rectified and filtered $\left(2^{\text {nd }}\right.$ order Butterworth with a low pass frequency cut-off of $10 \mathrm{~Hz}$ ) the data. The EMG was integrated (iEMG) from the time at which the participant received the signal to stop (trail-limb heel contact on the first force plate) for one second. The time of signal was chosen as the alignment point for the iEMG calculations to show any modifications that may occur before stepping on the rollers. One second of examination was chosen to include the reaction to the signal and the first step onto the rollers. 
Statistical analysis was performed using SPSS 14.0 for Windows ${ }^{\odot}$ (Chicago, Ill.). A one-way repeated measures design was used with the unexpected slippery stop, cued slippery stop trials 1 thru 5, and the average of the cued non-slippery stops to test for adaptive changes. Post-hoc analysis was done using Tukey’s tests $(\alpha=.05)$. Reported trends are based on qualitative observations and do not imply statistical significance.

\subsection{RESULTS}

\subsubsection{Kinetics}

Figure 3.2 shows the results of the peak and integrated braking force over the unexpected slippery stop, cued slippery stop trials and the average of the cued non-slippery stop trials. Both peak braking force and integrated braking force showed a significant trial effect (peak $\mathrm{F}=16.854, \mathrm{p}<.001$; integrated $\mathrm{F}=58.262, \mathrm{p}<.001$ ). The peak braking force significantly diminished after the unexpected slippery stop trial (cued slippery stop $1 \mathrm{p}=.004$, cued slippery stop $2 \mathrm{p}<.001$, cued slippery stop 3 and $4 \mathrm{p}=.001$, cued slippery stop5 $\mathrm{p}<.001$ ). The integrated braking force also significantly decreased compared to the slippery stop trial (cued slippery stop $1 \mathrm{p}=.012$, cued slippery stop $2-4 \mathrm{p}<.001$, and cued slippery stop 5 $\mathrm{p}=.001)$. The average of the cued non-slippery stop trials used significantly greater amounts of braking force compared to the cued slippery stop trials (peak braking force: all cued slippery stop trials $\mathrm{p}<.001$, integrated braking force: all cued slippery stop trials $\mathrm{p}<.001$ ). The integrated braking force was smaller in the unexpected slippery stop trial compared to 
the average cued non-slippery stop trials $(p<.001)$, but the peak braking force in the unexpected slippery stop trial was not different than the average cued non-slippery stop trials.

The changes in the peak braking force and braking impulse after the first slip suggests a change in the gait termination strategy. The propulsive forces (integrated or peak maximum) of the trailing limb showed no significant trial effect. The lack of change in the propulsive forces suggests the participants did not decrease propulsion to stop walking.

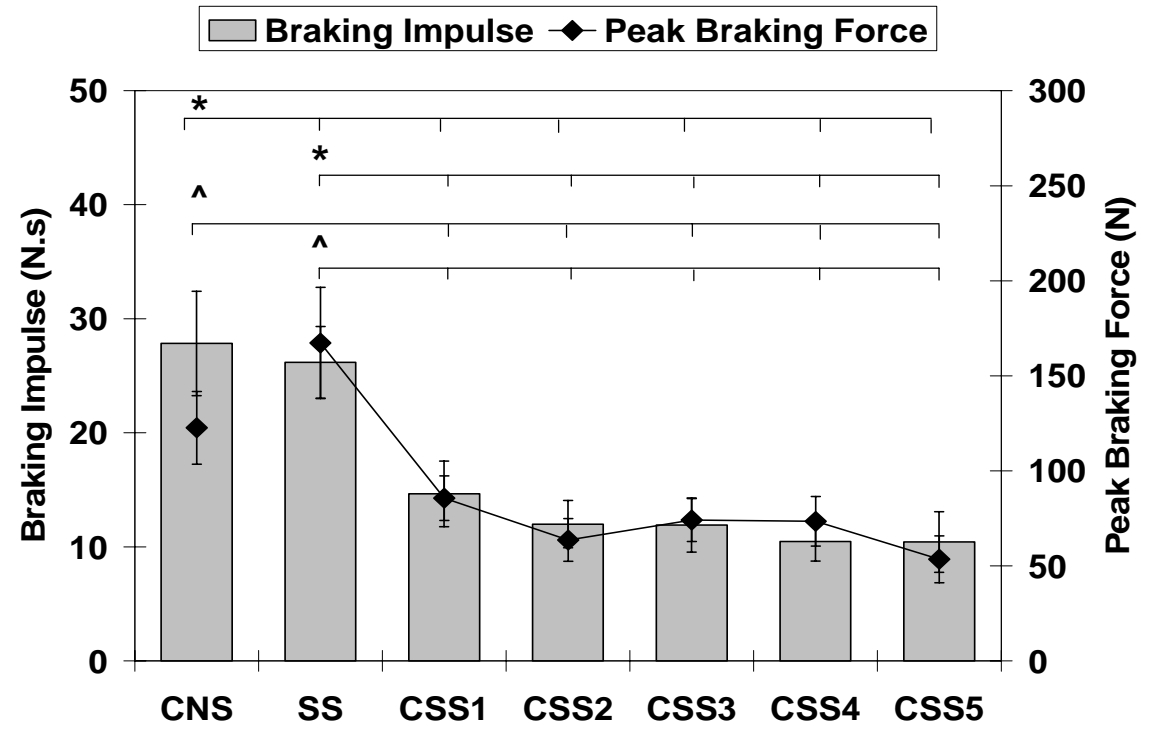

Figure 3.2: Average braking force impulse (left vertical axis) and peak braking force (right vertical axis) (+/- SE). * indicates significant difference in the average braking impulse from that trial and the other trials indicated by the vertical lines. $\wedge$ indicates significant difference in the peak braking force from that trial and the other trials indicated by the vertical lines. (CNS = average of the cued non-slippery stop trials, CSS = cued slippery stop trials, $\mathrm{SS}=$ unexpected slippery stop trial).

\subsubsection{Kinematics}

The approach velocity showed a significant trial effect $(\mathrm{F}=2.668, \mathrm{p}=.028)$. The first and second cued slippery stop trials were significantly slower than the unexpected slip (cued slippery stop $1 \mathrm{p}=.023$, cued slippery stop $2 \mathrm{p}=.027$ ) which suggests that after the first slip 


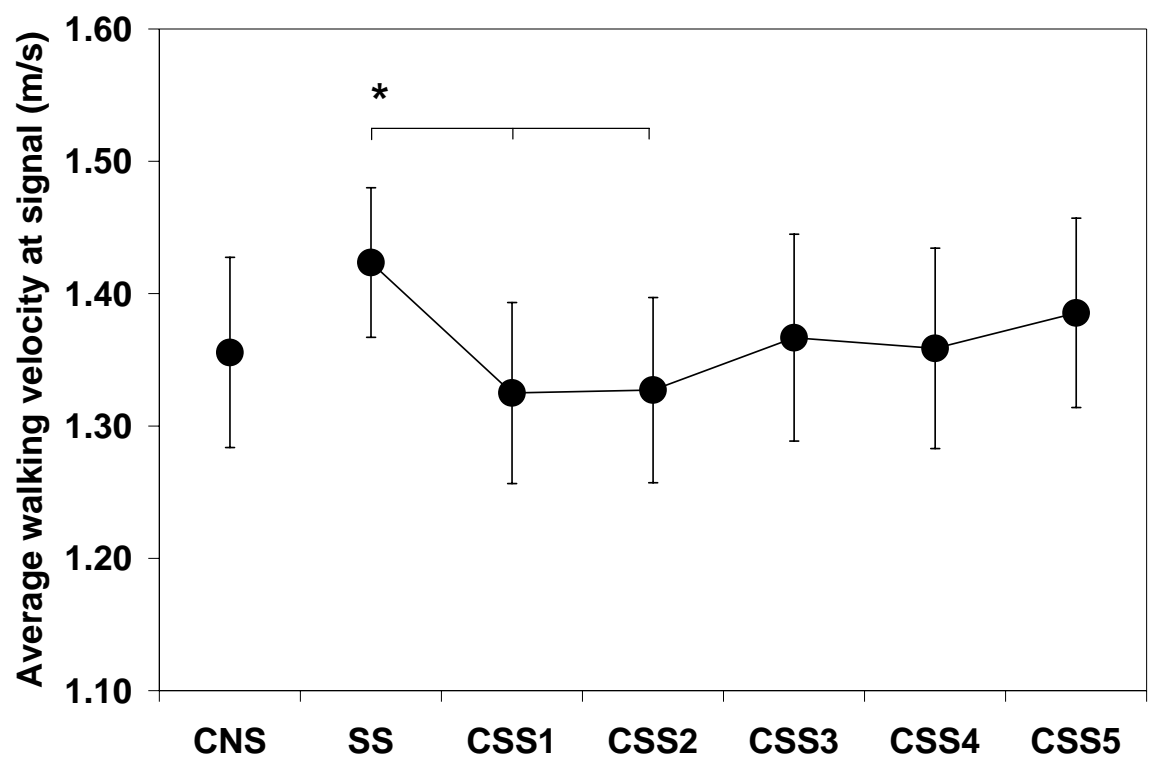

Figure 3.3: Average velocity one step before rollers (+/- SE). * indicates a significant difference between that trial and the other trials indicated by the vertical lines. (CNS = average of the cued, non-slippery stop trials, CSS = cued slippery stop trials, $\mathrm{SS}=$ unexpected slippery stop trial).

trial, participants slowed their walking but quickly returned to their pre-unexpected slip speed after the two cued slippery stop trials. Figure 3.3 shows the average approach velocity for the unexpected slippery stop, the cued slippery stop and average of the cued non-slippery stop trials. The time to slow showed a significant trial effect $(F=3.149, p=.012)$. The time to slow for the cued slippery stop 2 trial was earlier than the cued slippery stop $5(p=.005)$ but there is no trend over the trials. There was also a significant trial effect for the time to stop $(\mathrm{F}=3.004, \mathrm{p}=.016)$. No post-hoc differences were revealed between trials yet there is a trend of an increased time to stop in the cued slippery stop trials after the first unexpected slippery stop. 
Figure 3.4 shows the peak downward displacement in the COM during the time between heel contact on the rollers and $500 \mathrm{~ms}$ after heel contact. There was no significant trial effect in the vertical displacement of the $\operatorname{COM}(\mathrm{F}=1.192, \mathrm{p}=.329)$; however, plotting the data shows that participants dropped their COM lower in the slippery stop trial when compared to all of the cued slippery stop trials and the average of the cued non-slippery stop trials.

The average distance between the lead limb heel marker and the COM in the anterior-

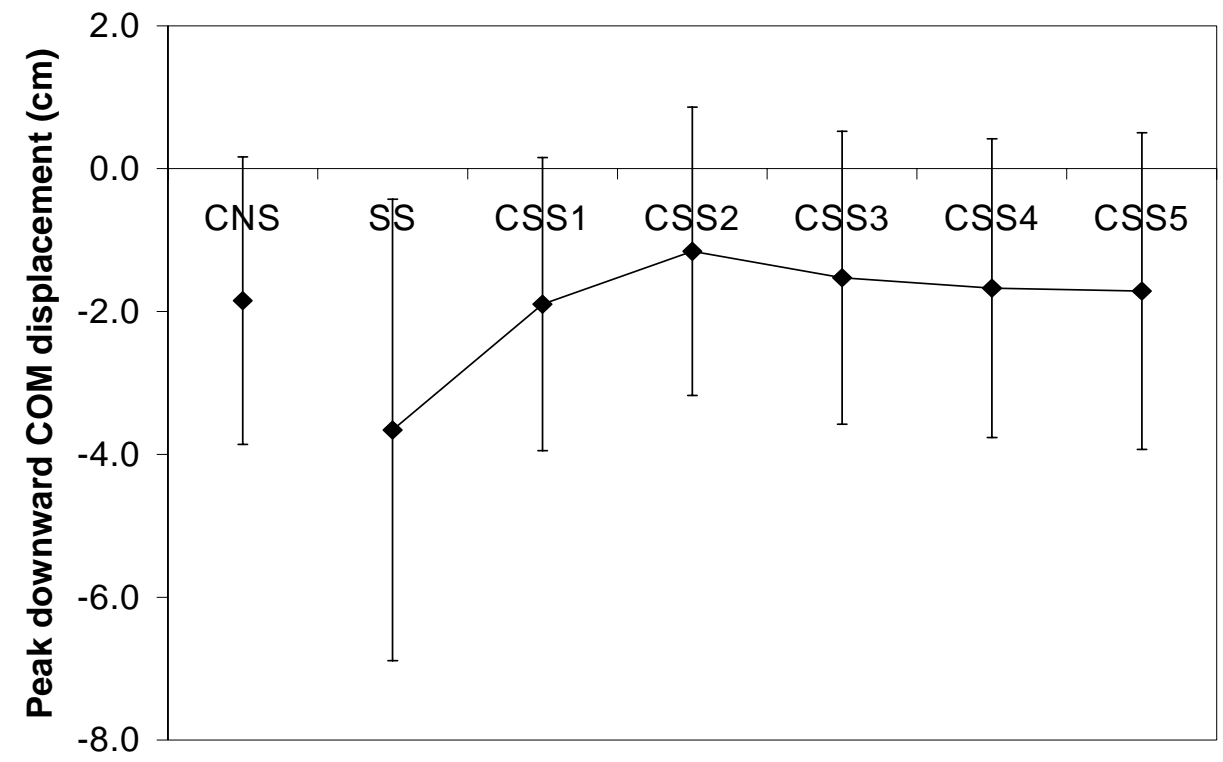

Figure 3.4: Peak downward displacement of the COM (+/- SE). Values are normalized to the average value during walk-through trials. (CNS $=$ average of the cued non-slippery stops, $\mathbf{S S}=$ unexpected slippery stop, $\mathrm{CSS}=$ cued slippery stop).

posterior plane is plotted in figure 3.5. The plot shows a significant decrease in distance between the heel and the COM over the cued slippery stop trials $(\mathrm{F}=5.286, \mathrm{p}<.001)$. Post-hoc analysis revealed an adaptation within two trials after the first unexpected slip: a significantly decreased distance between the lead heel marker and the COM in cued slippery stop 2 


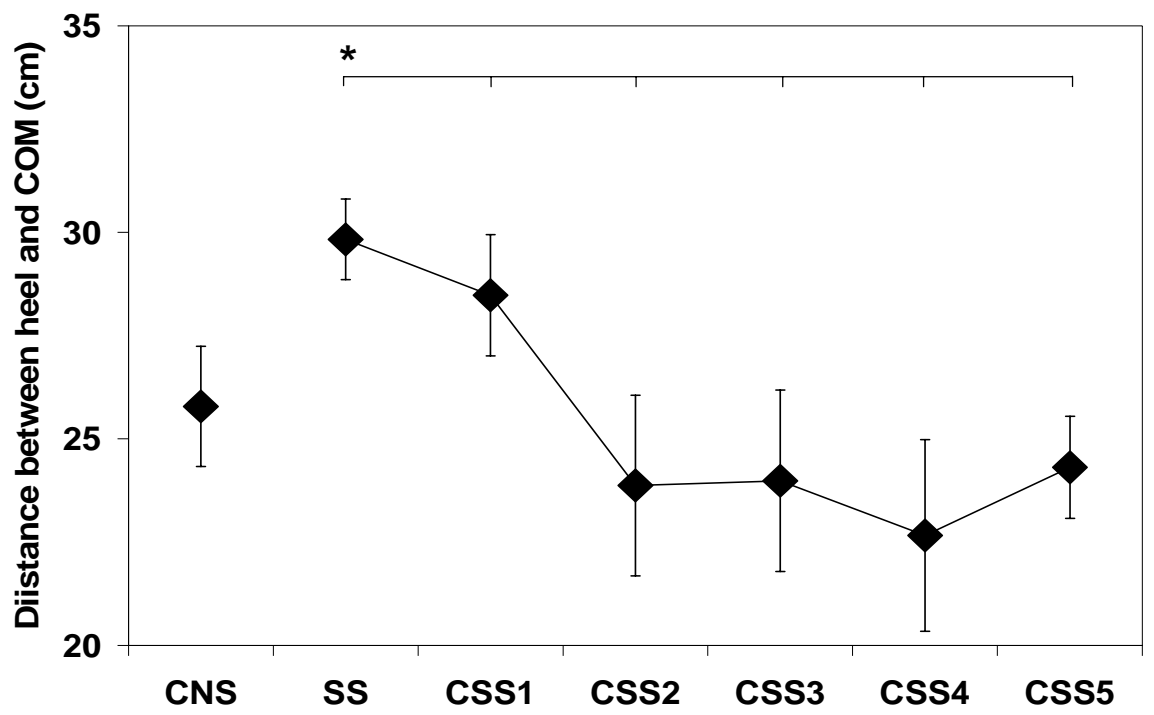

Figure 3.5: Average distance between the lead limb heel and the COM in the A-P direction at heel contact on the rollers $(+/-\mathrm{SE}) . *$ indicates a significant difference between that trial and other trials indicated by the vertical lines. (CNS $=$ average of the cued non-slippery stops, SS = unexpected slippery stop, CSS = cued slippery stop).

$(p=.012)$, cued slippery stop $3(p=.014)$, cued slippery stop $4(p=.001)$ and cued slippery stop $5(\mathrm{p}=.024)$ when compared to the unexpected slippery stop trial.

The angle of the foot at heel contact on the rollers significantly changed between trials $(\mathrm{F}=9.829, \mathrm{p}<.001)$, as shown in figure 3.6, and demonstrated an adaptation within two trials after the first, unexpected slippery stop. When compared to the slippery stop trial, the foot landed significantly flatter for all of trials except the first cued slippery stop trial (cued non-slippery stop average $\mathrm{p}=.001$, cued slippery stop $2-5 \mathrm{p}<.001$ ). The cued slippery stop 1 trial showed a significantly larger foot angle at heel contact than the cued slippery stop 2 $(p=.013)$, cued slippery stop $3(p=.016)$, and cued slippery stop $4(p=.007)$. The flatter foot after the unexpected slippery stop trial in subsequent trials including the non-slippery stops suggests an overly cautious foot-landing strategy for a non-slippery surface. 


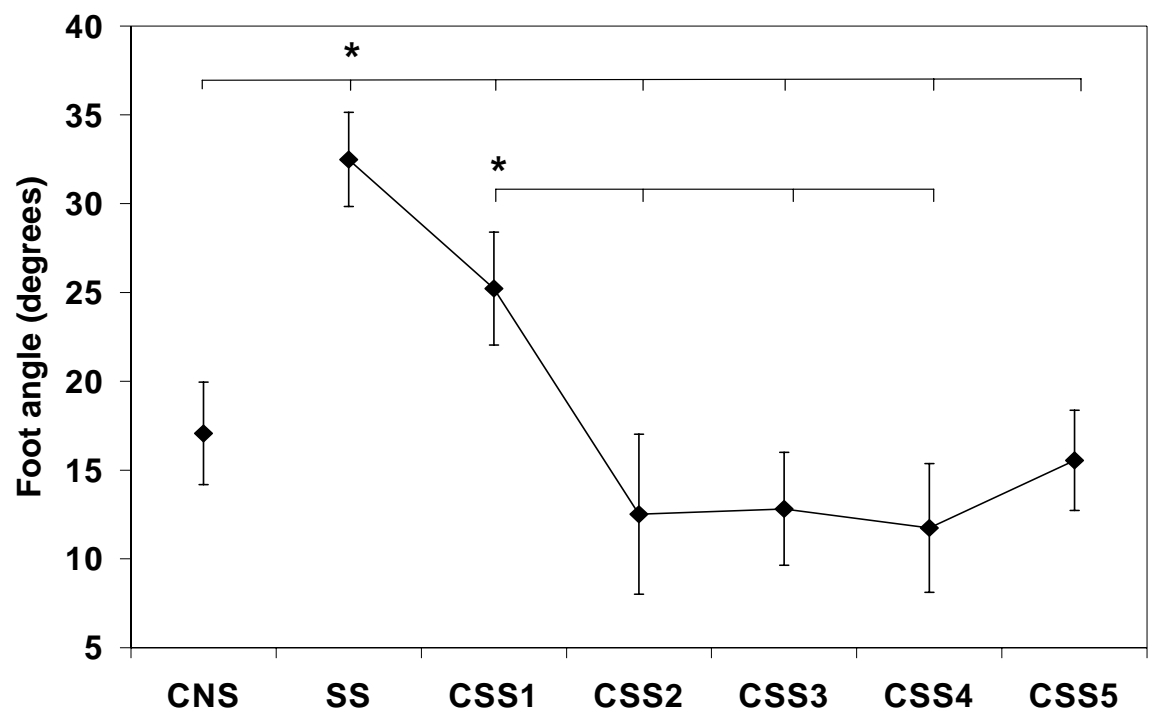

Figure 3.6: Foot angle at lead limb contact on the rollers (+/- SE). * indicates a significant difference between trials. (CNS = average of the cued non-slippery stops, SS = unexpected slippery stop, CSS = cued slippery stop).

Figure 3.7 shows the average heights of the right and left arm raises for all conditions.

There was a significant trial effect in both the left $(\mathrm{F}=5.400, \mathrm{p}<.001)$ and right $(\mathrm{F}=3.488$, $\mathrm{p}=.007)$ arms. The left arm was raised significantly higher in the unexpected slippery stop trial when compared to the cued slippery stop $2(p=.006)$, cued slippery stop $3(p=.027)$, cued slippery stop $4(p=.004)$ and the average of the cued non-slippery stop trials $(p<.001)$. The left arm was also higher in the first cued slippery stop trial compared to the average of the cued non-slippery stop trials $(\mathrm{p}=.048)$. Interestingly, the right arm raise was lower in the slippery stop trial compared to the cued slippery stop $1(\mathrm{p}=.009)$ and cued slippery stop 2 trials $(\mathrm{p}=.021)$. Thus the left arm showed adaptive changes within two cued slippery stop trials but the right arm did not as the response in the right arm became more like that seen in the first, unexpected slippery stop trial. 


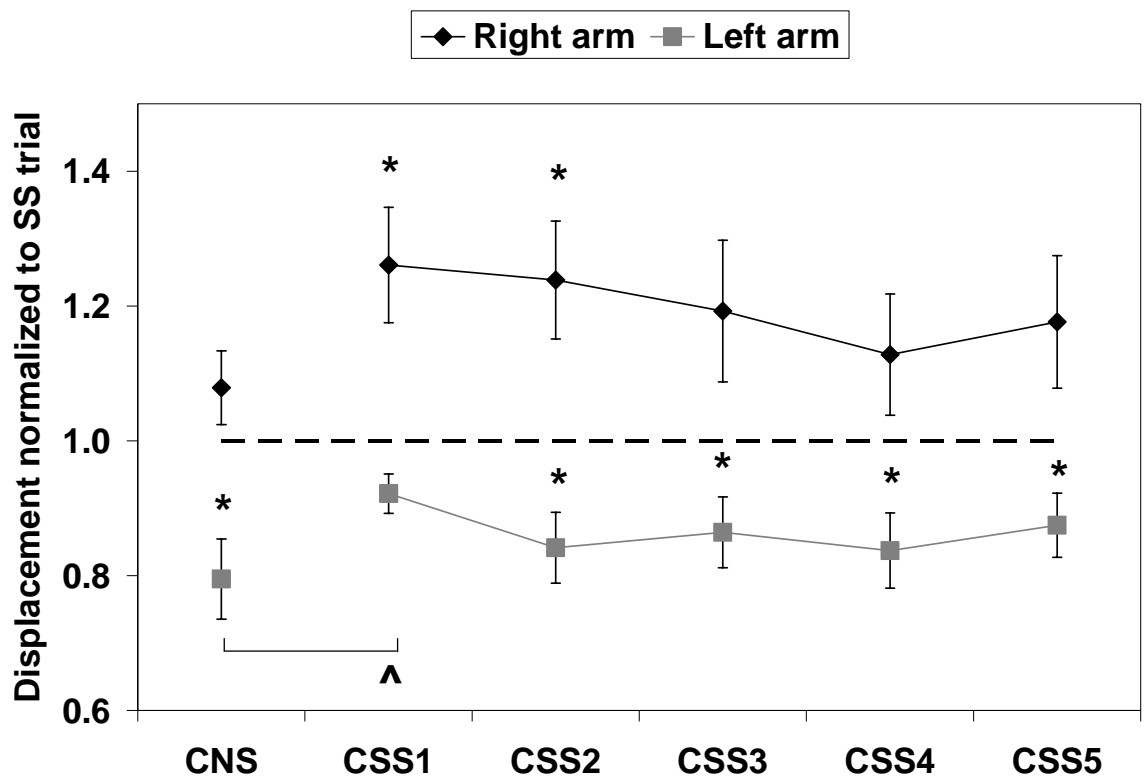

Figure 3.7: Peak arm raise height normalized to the unexpected slippery stop trial as indicated by the dashed line $+/-\mathrm{SE}$ (value $=$ trial/SS trial). * indicates a significant difference from the SS trial, ${ }^{\wedge}$ indicates a significant difference between trials. $(\mathrm{CNS}=$ average of the cued non-slippery stops, $\mathrm{SS}=$ unexpected slippery stop, CSS = cued slippery stop).

\subsubsection{Muscle response}

Figure 3.8 is a stacked plot of the average integrated EMG (iEMG) values for all conditions and both limbs. Table 3.1 displays all of the values displayed in figure 3.8. The values in Table 3.1 are normalized to the unexpected slippery stop trial (trial/SS trial = value). The lead limb SOL showed no significant trial effect $(\mathrm{F}=1.353, \mathrm{p}=.256)$. The TIB muscle activity demonstrated a significant trial effect $(F=11.026, p<.001)$ with a significant adaptation by the fourth cued slippery stop trial. The lead limb BF muscle also resulted in a significant trial effect $(\mathrm{F}=8.800, \mathrm{p}<.001)$ and was significantly different by the fourth cued slippery stop like the lead limb TIB. Activity in the lead limb RF muscles showed a significant trial effect $(\mathrm{F}=15.813, \mathrm{p}<.001)$ and with a significant decrease by the second cued 

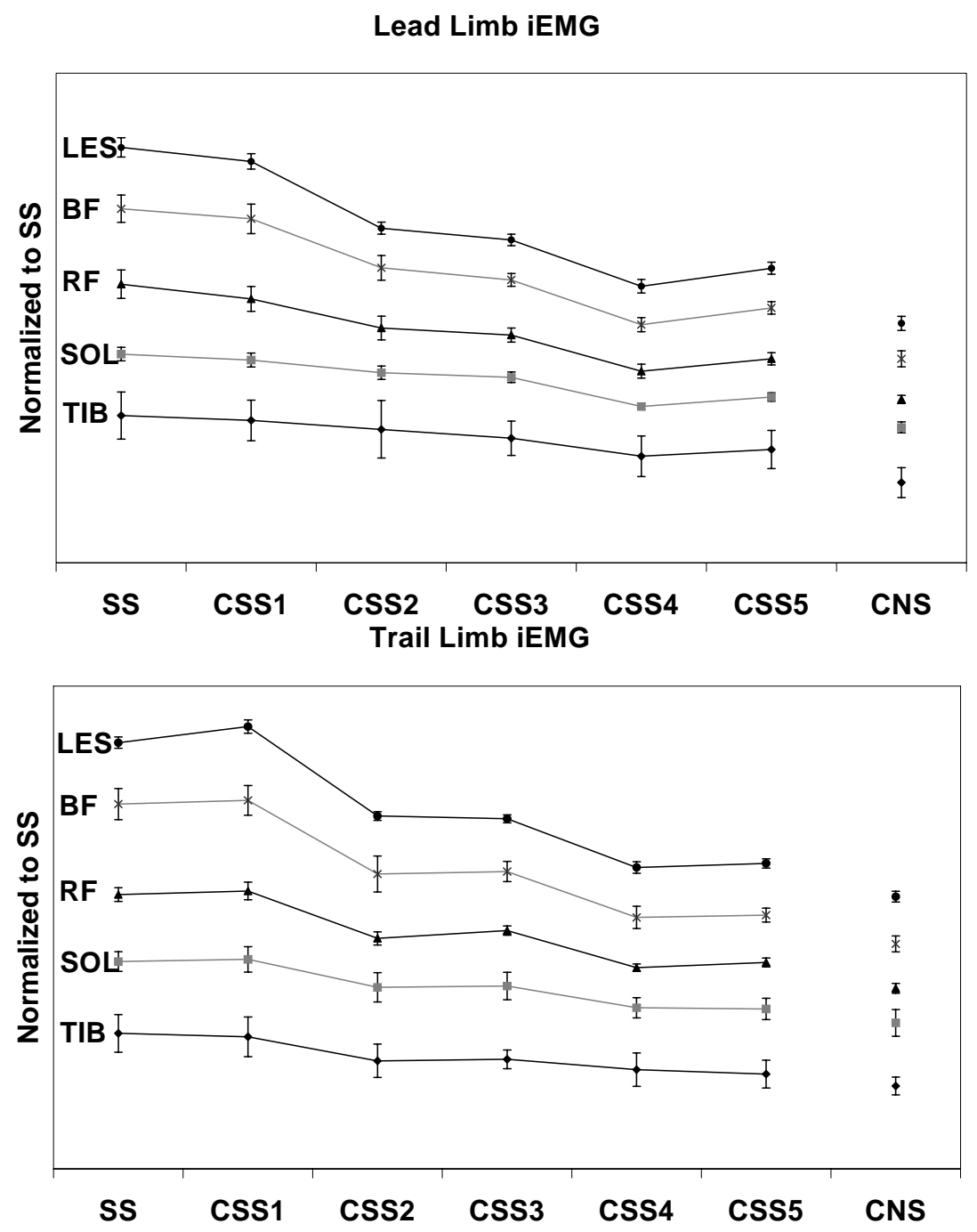

Figure 3.8: Stacked plots of the average integrated EMG trends for each muscle in the lead (top plot) and trail (bottom plot) limb (+/- SE). (CNS = average of the cued nonslippery stops, $\mathrm{SS}=$ unexpected slippery stop, $\mathrm{CSS}=$ cued slippery stop). All SS values are $=\mathbf{1 . 0}$.

slippery stop trial. A significant trial effect was seen in the lead limb LES muscle $(\mathrm{F}=11.311$, $\mathrm{p}<.001)$ and showed a similar pattern to the lead limb RF activity in that there was a significant adaptation by the second cued slippery stop. 
All of the trail limb muscles showed a significant trial effect (SOL $F=3.109, p=.013$; TIB F=6.098, $\mathrm{p}<.001 ; \mathrm{BF} F=12.756, \mathrm{p}<.001, \mathrm{RF} F=11.905, \mathrm{p}<.001$, and LES F=6.978, $\mathrm{p}<.001)$. The TIB and RF muscles showed adaptations by the fourth cued slippery stop trial. The BF significantly decreased its activity by the second cued slippery stop trial. Despite significant trial effects, the SOL and LES muscles did not show any significant decreases in activity compared to the first unexpected slippery stop.

\subsection{DISCUSSION}

With repeated exposure and information about the surface characteristics, participants adapted to stopping on the slippery surface. While some of the original slip response remained (arm raise and increased muscle activation), the magnitude of those responses diminished throughout the cued slippery stop trials and each participant was able to stop safely in all conditions. The movement pattern seemed to change from a reactive supportive strategy to a proactive surfing strategy where the COM remained elevated and the COM was

Table 3.1: Average iEMG values normalized to unexpected slippery stop trial (trial/SS trial) as shown in figure 3.8 .

\begin{tabular}{lrrrrrc}
\hline Muscle & CSS1 & CSS2 & CSS3 & CSS4 & CSS5 & CNS Avg. \\
\hline \hline RLES & 0.98 & 0.70 & 0.69 & 0.65 & 0.69 & 0.59 \\
RBF & 1.10 & 0.86 & 0.80 & 0.68 & 0.73 & 0.57 \\
RRF & 0.91 & 0.62 & 0.67 & 0.54 & 0.58 & 0.45 \\
RSOL & 0.98 & 0.94 & 1.04 & 0.86 & 0.91 & 0.94 \\
RTA & 0.99 & 0.87 & 0.88 & 0.72 & 0.77 & 0.56 \\
& & & & & & \\
LLES & 1.22 & 0.96 & 0.89 & 0.81 & 0.85 & 0.76 \\
LBF & 1.06 & 0.66 & 0.68 & 0.56 & 0.55 & 0.50 \\
LRF & 1.03 & 0.77 & 0.87 & 0.62 & 0.71 & 0.54 \\
LSOL & 1.05 & 0.99 & 1.00 & 0.85 & 0.90 & 0.86 \\
LTA & 1.00 & 0.83 & 0.88 & 0.74 & 0.72 & 0.65
\end{tabular}


positioned closer to the leading edge of the BOS. The adaptive changes occurred quickly, most often within one trial of the unexpected slippery stop, as summarized in table 3.1.

The ground reaction forces under the lead foot illustrate the reactive changes that occur with repeated exposure to the slippery surface. The decreased peak braking force and braking force impulse over the subsequent cued slippery stop trials demonstrates that the participants did not use the same strategy to stop on the slippery surface as in the first unexpected slip and in the cued non-slippery stop trials (Cham and Redfern 2002; Heiden et al 2006). With knowledge of the slippery surface, participants did not use an increase in braking force applied through the lead limb to stop walking and this change occurred immediately after the first slip experience. The similarity in the peak braking force for the unexpected slippery stop trial and the average cued non-slippery stop trials shows that participants attempted to use a similar braking strategy in the first slip exposure. The participants did not significantly decrease the amount of propulsive forces to stop on the slippery surface. The consistency in the propulsive force suggests that gait termination was facilitated by a means other than increased braking or decreased propulsion and that this parameter was not proactively manipulated to cope with the slippery surface.

The differences in the approach velocity demonstrate that participants proactively slowed their walking speed after the first, unexpected slip but then returned to pre-slip walking velocity with repeated trials. The initial slowing shows an increased cautiousness during those gait termination trials that used a slippery surface (Heiden et al 2006). The return to pre-first slip velocity demonstrates that participants were able to accommodate the 
slippery surface within two trials without slowing their walking velocity. The delayed time to slow in the final cued slippery stop trial suggests that participants felt confident in their ability to stop on the slippery surface and did not attempt to slow their forward progression earlier in order to aide gait termination.

A lowering of the COM is used in the first, unexpected slip (Oates et al 2005) but is not used in subsequent slip trials. This change in reactive behaviour shows that participants immediately altered how they stopped on the slippery surface, using the knowledge that the surface was slippery by elevating their COM (Marigold and Patla, 2002). Qualitative observations confirm these data as participants seemed to "surf" the rollers rather than lowering their bodies during the slip trials. The decreasing distance between the heel and the COM position in the anterior-posterior direction at heel contact shows that participants kept their COM closer to the lead limb during repeated trials on the slippery surface in a proactive manner; this adaptation also occurred early (after two experiences on the slippery surface). By keeping their COM close to the lead limb, the participant is able to move the COM within the newly-formed base of support faster (Bhatt, Wang and Pai 2006), and facilitate gait termination. This surfing strategy which is also seen in locomotion over a slippery surface (Marigold and Patla 2002) helps to keep the COM within the BOS during the slip until either the slip has ended or stability has been regained by stepping outside of the slippery surface (e.g., shortened subsequent step). 
Table 3.2: Summary table for dependent measures showing when adaptation occurred after the unexpected slippery stop trial (SS). Information in parentheses indicates when one trial was significantly different from the SS trial but the change did not persist across trials in that condition. No difference from the SS trial is indicated by n.d.

\begin{tabular}{lc}
\hline Dependent Measure & Trial adaptation occurred \\
\hline \hline Peak braking force & CSS1 \\
Braking impulse & CSS1 \\
Peak propulsive force & n.d. \\
Propulsive impulse & n.d. \\
Walking velocity & (CSS1 and CCS2<SS) \\
Time to slow & n.d. \\
Time to stop & n.d. \\
Peak downward COM movement & CSS1 \\
Distance between COM and lead heel & CSS1 \\
Angle of foot & CSS1 \\
Right arm raise & CSS2 \\
Left arm raise & \\
Lead limb iEMG: & n.d. \\
Soleus & CSS4 \\
Tibialis Anterior & CSS2 \\
Rectus Femoris & CSS4 \\
Biceps Femoris & CSS2 \\
Lower Erector Spinae & \\
Trail limb iEMG: & n.d. \\
Soleus & CSS4 \\
Tibialis Anterior & CSS4 \\
Rectus Femoris & CSS2 \\
Biceps Femoris & n.d. \\
Lower Erector Spinae &
\end{tabular}

A flatter foot is important in adapting to the slippery surface as shown by the decreasing foot-floor angle at contact on the rollers. The flattened foot allows for increased surface-area contact with the slippery surface and reduces the shear forces between the heel and the surface (Brady et al 2000; Cham and Redfern 2002; Heiden et al 2006; Marigold and Patla 2002; Marigold et al 2003). The significant change in the foot-floor angle shows that the participants were able to quickly (after the first cued slippery stop trial) alter their foot 
landing angle as a proactive measure to reduce the amount of slippage on the rollers. The presence of a flattened foot in the cued non-slippery stop trials suggests an overly cautious step onto the rollers and, perhaps, mistrust in the protocol (i.e., that the rollers were actually locked and not slippery) after being once deceived for the unexpected slippery stop. The awareness of the possibility of a slip occurring may have been enough of a stimulus to cause the adaptive behaviour in the absence of the slippery surface (Heiden et al 2006).

Any arm raise occurred after the slip was initiated and, therefore, is a reactive component of the motor program for gait termination on a slippery surface. When the left and right arm raises are examined separately, the left arm shows some early adaptation through a diminished arm raise with subsequent slip trials. The right arm also shows a change in behaviour after the first slip but, opposite to the left arm, the right arm raises higher in the first two cued slippery stop trials. The diminished response in the left arm could be due to an elimination of a startle response (Nieuwenhuijzen et al 2000), an attempt to create a smooth transition into gait termination (Marigold et al 2003; Nieuwenhuijzen et al 2000) or simply an increased comfort with the slippery surface (Marigold and Patla, 2002). The increased response in the right arm may be a pre-planned action to control stability while stopping on the slippery surface (Marigold et al 2003; Tang and Woollacott 1998a) in an attempt to increase stability on the side of the body which is experiencing the slip to aide in the smooth transition from a stabilizing response during the slip to stance. 
All of the muscles examined showed attenuation in the response over repeated cued slippery stop trials. The decrease in the response shows an adaptation to the slippery surface and an ability to use prior knowledge of the surface characteristics and influence this reactive element of the slip response (Marigold and Patla 2002). The sustained magnitude of the responses in both the lead and the trail limb ankle extensors suggests that these muscles are imperative in the gait termination program. The ankle extensors in both limbs are used to stabilize the ankle joints throughout GT regardless of the surface characteristics; the lead limb ankle extensors would be ineffective in generating a braking force on the rollers (as supported by the kinetic data from the force plates) but must have been required to generate sufficient force to stabilize the lead ankle during the slippery stops (Chambers and Cham 2006). An increased push-off from the trail limb would be inappropriate for any situation of gait termination; thus the trail limb ankle extensors must have functioned to provide stability to the ankle joint in the slippery and non-slippery stops.

The thigh and trunk musculature quickly diminished the magnitude of their response in the following cued slippery stop trials as the participant became more efficient at stopping on a slippery surface. The TIB for both limbs and the BF for the lead limb did not show an adaptation until the fourth cued slippery stop suggesting they are important in the slip recovery response and require repeated exposures to the slippery surface to change motor patterns. 
If the participants did not use an increase in braking force or a decrease in propulsive force to stop walking, how was gait termination completed? Each muscle increased its activity beyond what was used in the walk-through trials. The increase in muscle response would stiffen the joints in the lead/supporting limb and slow the forward movement of the swing limb which would help decrease the forward momentum of the body. The elevated COM in the cued slippery stop trials compared to the slippery stop trial also suggests that participants allowed their COM to remain elevated during the stops instead of dropping again as in steady-state locomotion. Finally, at heel contact on the rollers in the cued slippery stop trials, COM was held closer to the new base of support to allow a smooth transition from steady-state gait to gait termination despite the slippery surface.

A limitation to this study involves the external validity of the slip on the rollers. The rollers used to cause the slip are one-dimensional and, therefore, do not fully imitate a slip in a real-world environment which would allow a slip in both horizontal planes (Troy and Grabiner 2006). In addition, participants could have used the wooden platform at the end of the rollers to aid in their recovery from the slip whereas, on real ice or on a contaminated surface in a workplace, the boundaries of the slippery surface may be too large to use the edges in the slip recovery (Troy and Grabiner 2006). While the constraints of the laboratory potentially facilitated the slip recovery, we are confident that we were able to elicit a genuine slip response which can be generalized to every day slips during gait termination.

In conclusion, the ability of young, healthy adults to stop on a slippery surface quickly adapts within two exposures and becomes more efficient with knowledge of the 
surface characteristics and repeated exposure. Most of the proactive and reactive changes in behaviour occur immediately after the unexpected slippery stop with the exception of the left arm (took two trials to show an adaptation) and the lower limb musculature (took two to four trials to show an adaptation). The lead limb and the distal segments especially seem to have the most influence on the gait termination and slip recovery responses in maintaining balance and stopping safely on a slippery surface as their responses took the longest to show a change in the motor program for gait termination on a slippery surface. 


\subsection{REFERENCES}

Bhatt T, Wang E, and Pai Y-C. (2006). Retention of adaptive control over varying intervals: Prevention of slipinduced backward balance loss during gait. J. Neurophysiol, 95: 2913-2922.

Brady RA, Pavol MJ, Owings TM and Grabiner MD. (2000). Foot displacement but not velocity predicts the outcome of a slip induced in young subjects while walking. J. Biomech, 33: 803-808.

Cham R and Redfern MS (2001). Lower extremity corrective reactions to slip events. J. Biomech, 34: 14391445.

Cham R, and Redfern MS. (2002). Changes in gait when anticipating slippery floors. Gait Posture, 15: 159-171.

Chambers AJ and Cham R. (2006). Slip-related muscle activation patterns in the stance leg during walking. Doi:10.1016/j.gaitpost.2006.06.007.

Ferber R, Osternig LR, Woollacott MH, Wasielewski NJ, and Lee J. (2002). Reactive balance adjustments to unexpected perturbations during human walking. Gait and Posture, 16: 238-248.

Heiden TL, Sanderson DJ, Inglis JT, and Siegmund GP. (2006). Adaptations to normal human gait on potentially slippery surfaces: The effects of awareness and prior slip experience. Gait Posture, 24: 237-246.

Henry SM, Fung J, and Horak FB. (1998). EMG responses to maintain stance during multidirectional surface translations. J. Neurophysiol., 80: 1939-1950.

Marigold DS, and Patla AE. (2002). Strategies for dynamic stability during locomotion on a slippery surface: effects of prior experience with and knowledge about surface characteristics. J Neurophysiol, 88: 339-353.

Marigold DS, Bethune AJ, and Patla AE. (2003). Role of the unperturbed limb and arms in the reactive recovery response to an unexpected slip during locomotion. J Neurophysiol, 89: 1727-1737.

Misiaszek JE, Stephens MJ, Yang JF, and Pearson KG. (2000). Early corrective reactions of the leg to perturbations at the torso during walking in humans. Exp Bran Res, 131: 511-523.

Misiaszek JE. (2003). Early activation of arm and leg muscles following pulls to the waist during walking. Exp. Brain Res., 151: 318-329.

Myung R and Smith JL. (1997). The effect of load carrying and floor contaminants on slip and fall parameters. Ergonomics, 40 (2): 235-246.

Nieuwenhuijzen PHJA, Schillings AM, Van Galen GP, and Duysens J. (2000). Modulation of the startle response during human gait. J Neurophysiol, 84: 65-74.

Oates AR, Patla AE, Frank JS, and Greig MA. (2005). Control of dynamic stability during gait termination on a slippery surface. J Neurophysiol, 93:64-70.

Tang P and Woollacott MH. (1998a). Inefficient postural responses to unexpected slips during walking in older adults. J Gerontology: Med. Sci., 53A (6): M471-M480.

Tang P, Woollacott MH, and Chong RKY. (1998b). Control of reactive balance adjustments in perturbed human walking: roles of proximal and distal postural muscle activity. Exp Brain Res., 199: 141-152.

Troy KL and Grabiner. (2006). Recovery responses to surrogate slipping tasks differ from responses to actual slips. Doi:10.1016/j.gaitpost.2005.09009. 
CHAPTER 4: CONTROL OF DYNAMIC STABILITY DURING GAIT TERMINATION ON A SLIPPERY SURFACE WITH PARKINSON'S DISEASE 


\subsection{INTRODUCTION}

Gait termination is a challenging task requiring control of the forward momentum of the body's centre of mass (COM). This forward momentum must be dissipated and the COM held within a newly formed base of support. Stopping involves increasing the amount of braking force to slow forward progression and decreasing the amount of propulsive force used to move the body forward (Hase and Stein 1998; Jaeger and Vanitchatchavan 1992). If these strategies are unsuccessful, other movements such as another step or a rise-to-toes (Hase and Stein 1998) may be taken in order to stop safely. This voluntary change in motor programs, from walking to stopping, may pose a problem to people with Parkinson's disease (PD) who have difficulties switching between tasks (Abbruzzese and Berardelli 2003).

Typical Parkinsonian gait (a stiff, shuffling, slow walk) can include festination when attempting to stop (Bloem and Bhatia 2004; Morris et al 2001). This festination suggests a poor control over the ability to stop within one or two steps. Research shows that traditional dopamine replacement therapy can restore Parkinsonian gait to more normal speeds and stride-length but may cause more instability due to increased mobility and variability caused by the medication (Horak et al 1996; Morris 2006; Morris et al 2001). The need for a voluntary change in motor program along with difficulty stopping makes gait termination a potentially difficult task for someone with Parkinson's disease.

The instability seen in PD in response to an external perturbation is not corrected with medication (Carpenter et al 2004; Horak et al 1996). A slip during gait termination, therefore, would pose an even greater threat to someone with PD than gait termination alone. A slip 
during a stop requires the integration of a balance-recovery response into the voluntary gait termination program. Past research on automatic postural responses shows that the relative timing of balance-correcting movements is unaffected by PD despite decreased force output (Carpenter et al 2004; Dimitrova et al 2004; Horak et al 1996; Morris et al 2001; Nieuwboer et al 2004). Thus, the response of someone with PD should occur within the same time period as a healthy control but may be diminished in magnitude.

The purpose of this study is to investigate how PD affects the ability to switch motor programs from a steady-state locomotor pattern to gait termination (GT) and to examine the ability of someone with PD to integrate a reactive, balance maintenance strategy into a planned GT program. Comparing persons with PD to age-matched controls during planned and cued GT on both a stable and a moving surface will illustrate the effect of PD on the GT motor program and the balance recovery response. We hypothesize that PD will negatively affect the ability to quickly change motor programs in that the PD group will take longer to stop and will be less stable than the control group in the non-slippery conditions. We also hypothesize that, due to the lack of protection from an external perturbation while on medication, the PD group will be less stable than the control group while stopping on a slippery surface. 
Table 4.1: Age, motor UPDRS score, and Hoehn and Yahr score for PD participants

\begin{tabular}{cccc}
\hline ID & Age & UPDRS & H \& $\mathbf{~}$ \\
\hline \hline 1 & 71 & 31.5 & 2.5 \\
2 & 68 & 36 & 2 \\
3 & 67 & 40 & 3 \\
4 & 62 & 42.5 & 2 \\
5 & 51 & 36.5 & 2 \\
6 & 78 & 44 & 3 \\
7 & 63 & 7 & 1 \\
8 & 73 & 29 & 2 \\
9 & 62 & 24 & 2
\end{tabular}

\subsection{METHODS AND MATERIALS}

\subsubsection{Protocol}

Nine participants with idiopathic PD (age 66.3 +/- 7.3 years SD) and ten age-matched controls (age 65.4 +/- 7.3 years SD) participated in this study (see table 1 for PD participant characteristics). All PD participants were on their usual medication during testing. Before data collection, a modified Unified Parkinson's Disease Rating Scale (UPDRS) examination was administered to examine the motor capabilities of individuals with PD where a higher score represents more disability. The severity of Parkinsonism, as measured by the Hoehn and Yahr scale ranged from 1 to 3 . All participants were able to walk independently and reported that they were free of orthopaedic, psychological, or other neurological disorders which could affect their ability to perform the tasks. A safety harness was worn throughout data collection. All participants provided written, informed consent for protocols approved by the Institutional Review Board for OHSU which stated that the plate may unexpectedly 
move underneath their feet when stepped on. Approval for this protocol was also obtained through the University of Waterloo's Office of Research Ethics.

All participants experienced four types of trials: 1) Walk-through trials were when the participant walked to the end of the room towards a set of lights. The lights were round, approximately $10 \mathrm{~cm}$ in diameter, resembled the red brake lights on a small motor vehicle and were located at eye level at the far end of the room. These trials provided baseline gait information and allowed the participant to become familiar with the laboratory set-up. 2) Planned non-slippery stop trials involved stopping on stationary force plates in the middle of the room. The lights at the end of the room were illuminated the entire time of the trial and the participants knew from the beginning of the trial that they were going to stop on the force plates. The planned non-slippery stop trials show how PD affects the ability to generate a stop over multiple steps. 3) Non-slippery stop trials involved cued GT on the same stationary force plates. The non-slippery stop trials were randomly placed throughout a series of 20 trials. The lights at the end of the room were controlled by placing an infrared light beam positioned at left foot contact (one step) before reaching the force plates. When the light beam was interrupted during the non-slippery stop trials, the lights illuminated. The participants were informed that if the lights illuminated then they were to stop with both feet on the force plates. Participants were not told to try to step on the force plates but to walk normally towards the end of the room. Their starting location was manipulated so that they would step on the right force plate with their right foot. The participant received the signal to stop on the left limb (here after referred to as the trail limb), stepped on a force plate with the 
right limb (here after referred to as the lead limb) and then finished gait termination with the trail limb on a force plate next to the lead foot. If the lights did not illuminate then they were to continue walking to the end of the room. The non-slippery stop trials show how PD affects the ability to stop within one step.

The final trial condition was 4) the slippery stop trial. The slippery stop trial immediately followed the series of non-slippery trials and participants were given the same instructions as the non-slippery stop trials. When the participant stepped on the first force plate (always with the lead foot) the plate moved forward rapidly and unexpectedly, mimicking a slippery surface. The plate movement was controlled using a force-integration function where the plate continued to accelerate until it reached its maximum displacement (an average of $14.7 \mathrm{~cm}$ forward at $0.47 \mathrm{~m} / \mathrm{s}$ ). This perturbation is similar in displacement but slower in slip velocity to previous investigations of stopping on a slippery surface (Oates et al 2005). The slippery stop trials show how PD affects the ability to integrate a balancecorrecting response to an external perturbation while stopping within one step.

\subsubsection{Measurement}

Kinetic data were captured from custom-made force plates using a QNX data collection system ( sampling rate $=480 \mathrm{~Hz}$ ) and used for identifying foot contact on the moving force plate. A high-resolution Motion Analysis System (Santa Rosa, CA) with 7video cameras, sampling at $60 \mathrm{~Hz}$, provided 3-dimensional spatial coordinate information about body segment displacements. Markers were placed on multiple anatomical landmarks bilaterally including the ear, acromion process, olecranon, styloid process, anterior superior 
iliac crest, greater trochanter, lateral femoral condyle, lateral malleolus, heel and fifth metatarsal, and on the xyphoid process.

\subsubsection{Analysis}

Step length and width were calculated from the heel markers of both feet. Step length was defined as the anterior-posterior (A-P) distance from the trail limb heel marker to the lead limb heel marker in subsequent steps. Step width was defined as the absolute mediallateral (M-L) distance between heel markers in subsequent steps. A 12-segment centre of mass $(\mathrm{COM})$ model was calculated using a custom-designed MATLAB program (Mathworks, Natick, MA) with marker data low-pass filtered at 6Hz. COM velocity was calculated by differentiating the COM displacement. The time to slow was defined as the time when the COM velocity in the anterior-posterior plane fell below the average minus two standard deviations of the average forward velocity of the COM during the walk-through trials. The time to stop was defined as the time when forward velocity fell within the average plus two standard deviations of the average velocity (over 500ms) one second after the right heel contact on the first force plate of the planned non-slippery stop trials. The time to slow represents the beginning of gait termination; the time to stop represents the completion of gait termination.

A margin of dynamic stability was calculated using the method developed by Hof et al (2005) which calculates an extrapolated COM position (xCOM) using the equation: $\mathrm{xCOM}$ $=\mathrm{COM}$ position $+\mathrm{COM}$ velocity $/ \sqrt{ }(\mathrm{g} / \mathrm{l})$, where $\mathrm{g}=$ acceleration due to gravity and $\mathrm{l}=$ vertical height of the COM. The position of the $\mathrm{xCOM}$ was compared to the position of the 
marker on the fifth metatarsal on the foot that was stepping (i.e., to the right foot during the first step of GT and to the left foot during the second step of GT) at the time of foot contact. This analysis incorporates both the relative position of the sagittal COM with respect to the foot and the velocity of the COM. The magnitude of the difference in the horizontal planes is the dynamic margin of stability.

Data were entered into a $2 \times 2$ (group $\mathrm{x}$ condition) ANOVA to compare average values between the non-slippery stop trials (planned and cued), and non-slippery stop and slippery stop (both cued) trials as separate analyses. Significance level was set at $\alpha=0.05$.

\subsection{RESULTS}

\subsubsection{Planned versus Cued non-slippery stops}

The PD group walked significantly slower than the control group with mean values of $1.259 \mathrm{~m} / \mathrm{s}$ for the control group and $0.916 \mathrm{~m} / \mathrm{s}$ for the PD group. The slowness in the PD group is representative of the bradykinetic nature of Parkinson's disease. The forward velocity of the COM at heel contact before GT (the last step before the force plates) was similar within groups for both conditions. Figure 4.1 illustrates the average heel contact locations for both groups in all three conditions (controls are represented by dark squares, PD by light squares). For the first step of GT onto the force plates the PD group showed a significantly shorter step in both the planned and the cued non-slippery stops $(\mathrm{F}=8.380, \mathrm{p}$ $=0.007)$. PD also had a wider step for the first step of GT onto the force plates in both planned and cued non-slippery stops $(\mathrm{F}=17.462, \mathrm{p}<0.005)$. 

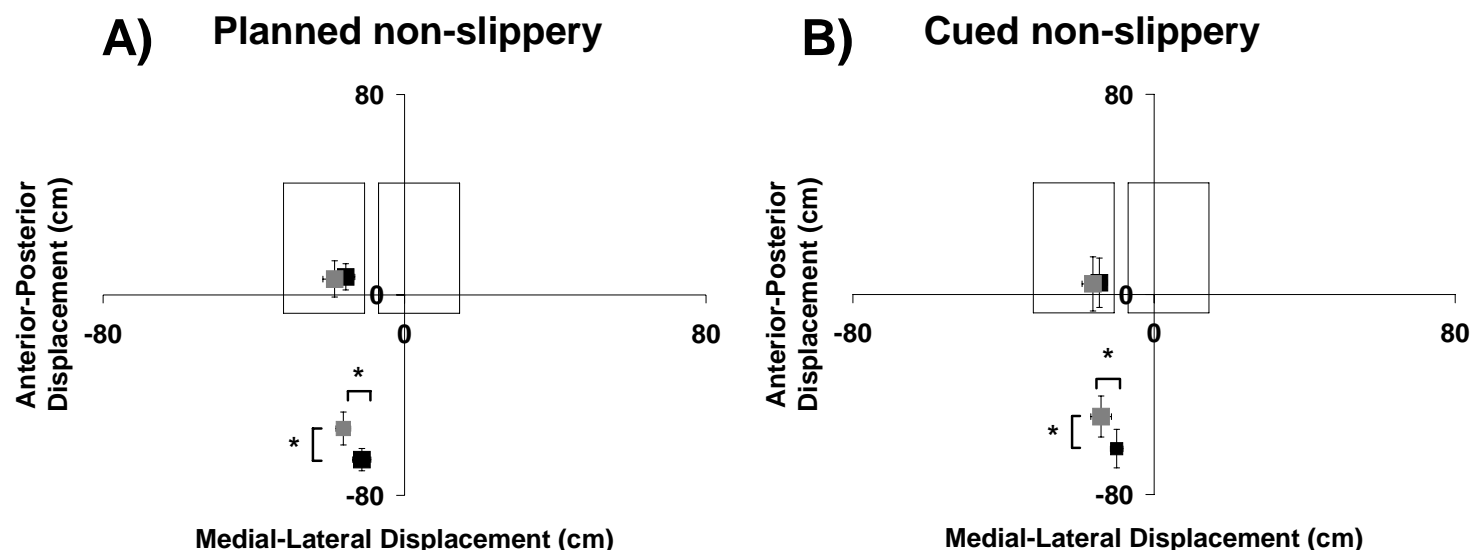

C) Slippery stop left heel contact

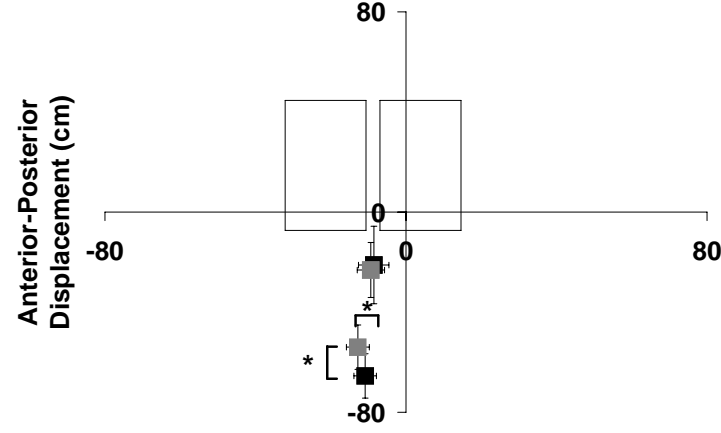

Medial-Lateral Displacement (cm)

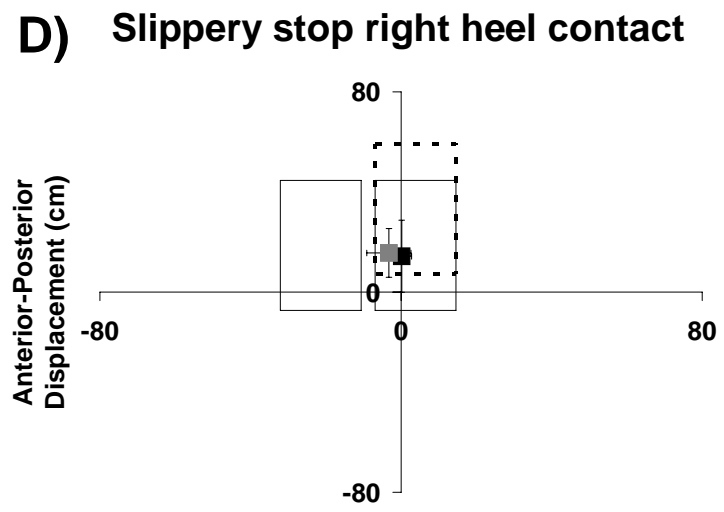

Medial-Lateral Displacement (cm)

Figure 4. 1: Comparisons for step length and width for PD and control groups. The rectangles on the plots are the position of the force plates; in figure $D$, the dashed rectangle is the final position of the moving right force plate. $(0,0)$ is the location of the lead heel contact on the force plate. The squares (dark are control, light are PD; error bars are SD) show the location of the left heel contact in A-C and right heel contact after the force plate has moved forward in $D$. The squares closest to $(0,0)$ are the terminal position of the left heel (A-C) or right heel (D). The lower squares in $\mathrm{A}-\mathrm{C}$ are the location of the left heel contact preceding gait termination. * indicates a significant difference between groups (vertical line $=$ significant difference in step length, horizontal line = significant difference in step width).

The margin of dynamic stability during GT of both groups in all three conditions is

illustrated in figure 4.2. The control group was significantly more stable than the PD group (i.e., had a larger margin of stability) in the anterior-posterior (A-P) plane in the first step of non-slippery GT $(\mathrm{F}=8.365, \mathrm{p}=0.007)$. Both groups were significantly more stable in the medial-lateral (M-L) plane during the first step for the planned stop trials compared to the 


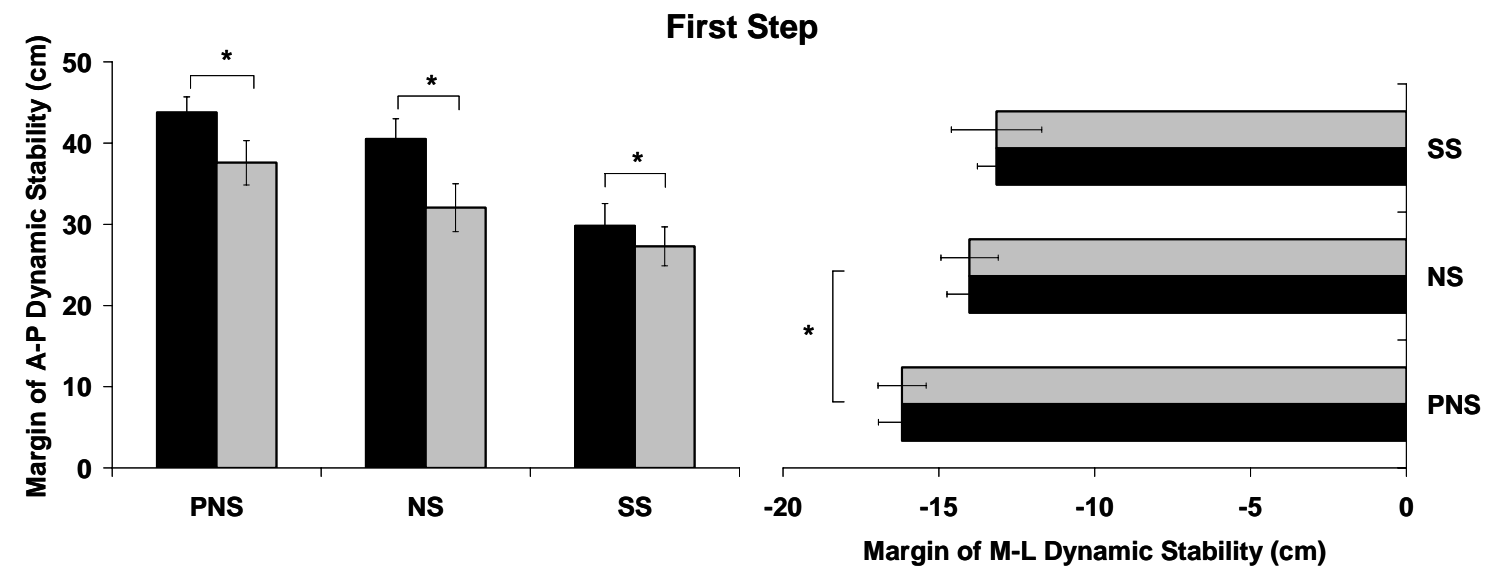

Second Step
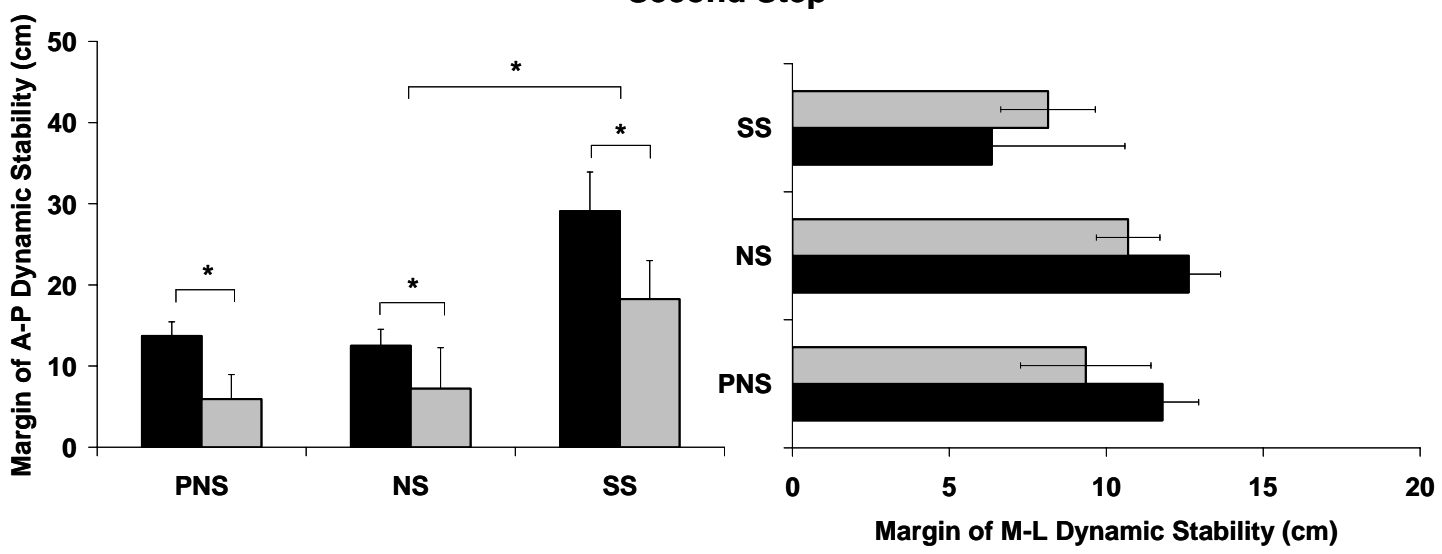

Figure 4.2: Dynamic stability during the first and second step of gait termination (+/SE). * indicates a significant difference between groups or trials. The control group is represented by the dark bars, the $\mathrm{PD}$ group is represented by the light bars. (PNS = planned slippery stops, NS = cued non-slippery stops, SS = unexpected slippery stop). cued non-slippery stop trials $(\mathrm{F}=8.193, \mathrm{p}=0.007$,). In the final step of $\mathrm{GT}$, the control group was more stable than the PD group in the A-P plane $(F=4.361, \mathrm{p}=0.044)$ but there was no difference between groups in the $\mathrm{M}-\mathrm{L}$ plane $(\mathrm{F}=2.575, \mathrm{p}=0.118)$.

Both groups were able to slow and stop with similar timing for the planned and the cued non-slippery stop. Mean values of the time to slow for the control and PD groups were $215 \mathrm{~ms}$ and $224 \mathrm{~ms}$ after the signal was (cued stop) or would have been (planned stop) given. 

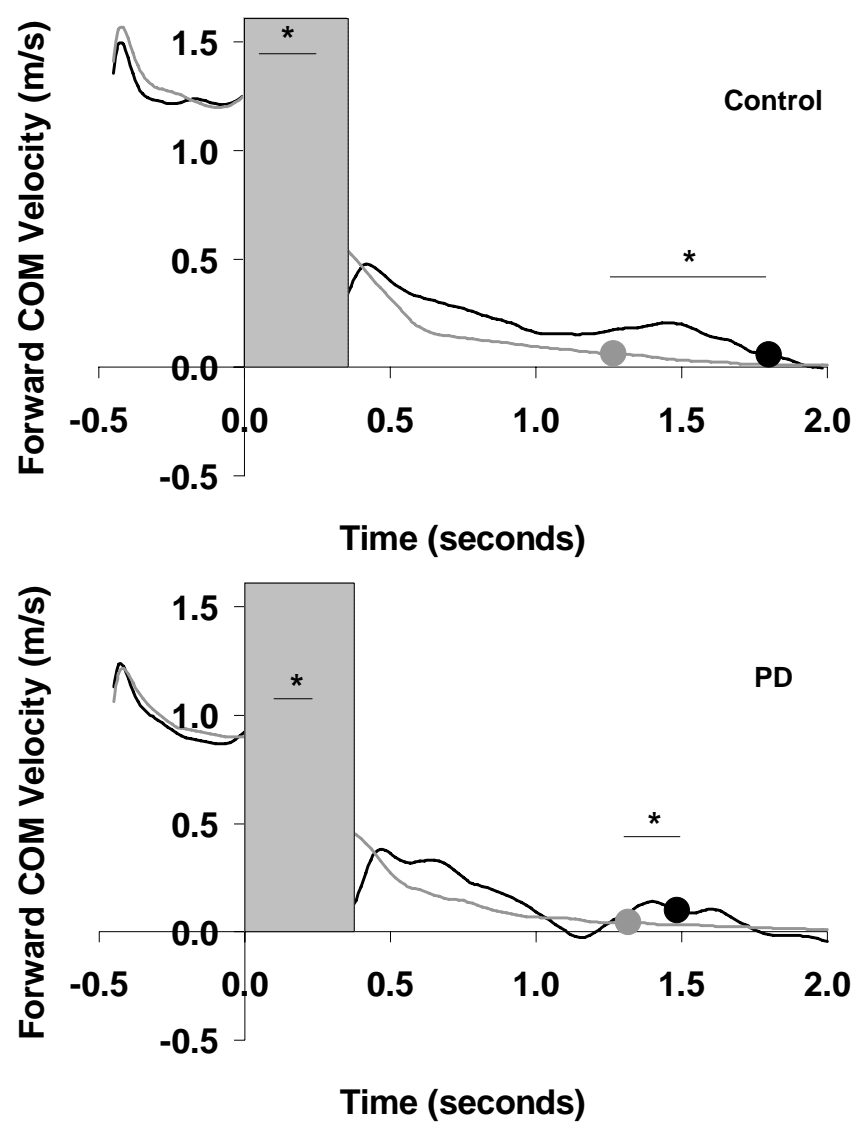

Figure 4.3: Forward COM velocity tracings showing time to slow and time to stop in representative control and $P D$ participants. The triangle-shape represents time to slow, the circle represents time to stop. Time of zero seconds is heel contact on the force plate. The light lines represent the cued non-slippery stop trials, the dark lines represent the unexpected slippery stop trial. The shaded area is the time during which the platform moved forward. * indicates a significant difference of the group averages between conditions.

Mean values of the time to stop for the control and PD groups were $945 \mathrm{~ms}$ and $946 \mathrm{~ms}$ after the signal was (cued stop) or would have been (planned stop) given.

\subsubsection{Non-slippery versus slippery stops}

The PD group walked significantly slower than the control group $(\mathrm{F}=31.428, \mathrm{p}$ $<0.005$ ) with mean values for the PD and control groups of $0.953 \mathrm{~m} / \mathrm{s}$ and $1.291 \mathrm{~m} / \mathrm{s}$ 
respectively. The forward velocity of the COM at heel contact before GT (the last step before the force plates) was similar for both conditions within each group. For the first step of GT onto the force plates the PD group showed a significantly shorter step in both the nonslippery and slippery stop conditions $(\mathrm{F}=6.010, \mathrm{p}=0.02)$ (figure 1$)$. PD also had a wider step for the first step of GT onto the force plates in both the non-slippery and slippery stop conditions $(\mathrm{F}=7.121, \mathrm{p}=0.012)$. Step length was significantly longer in the final step for slippery stop trials in both groups when compared to the non-slippery stop trials $(\mathrm{p}<0.005, \mathrm{~F}$ $=18.195)$. There was no significant difference between groups for the step width in the final step of the slippery stop trial $(\mathrm{F}=0.998, \mathrm{p}=0.327)$.

The dynamic stability during GT of both groups in all three conditions is illustrated in figure 4.2. The control group was significantly more stable than the PD group (i.e., had a larger margin of stability) in the anterior-posterior $(\mathrm{A}-\mathrm{P})$ plane in the first $(\mathrm{F}=4.275, \mathrm{p}=.046)$ and second $(\mathrm{F}=3.517, \mathrm{p}=.069)$ step of GT in the non-slippery and slippery cued GT conditions despite a slightly higher p-value in the second step results. The final step of the slippery stop trial was more stable (had a larger stability margin) than the final step for the non-slippery stop trials in the A-P plane for both groups $(\mathrm{F}=10.301, \mathrm{p}=0.003)$. The increased margin of dynamic stability in the slippery stop trials is likely due to the increased forward displacement of the foot caused by the anterior displacement of the force plate. Similar to the planned non-slippery stop trials, there was no difference between groups in the M-L plane for the slippery stop trial (first step $F=0.607, p=0.441$; final step $F=0.001$, $p$ $=0.978)$. 
All participants slowed significantly earlier during the slippery stop trials $(\mathrm{F}=5.620$, $\mathrm{p}=0.024)$ but stopped later during the slippery stop trials $(\mathrm{F}=18.041, \mathrm{p}<0.005)$ when compared to the non-slippery stop trials (figure 4.3). When aligned with $\mathrm{HC}$ on the right force plate, the mean time to slow for both groups was $0.209 \mathrm{~ms}$ (non-slippery stop) and $0.089 \mathrm{~ms}$ (slippery stop). The mean time to stop for both groups was $0.905 \mathrm{~ms}$ (non-slippery stop) and $1.280 \mathrm{~ms}$ (slippery stop).

\subsection{DISCUSSION}

\subsubsection{Planned versus cued non-slippery stops}

Congruent with past research on persons with Parkinson's disease (Bishop et al 2006; Bloem and Bhatia 2004; Morris et al 2001), this study found that the PD group walked slower and had shorter steps than the age-matched controls. This finding was significant despite the PD group being on their dopamine replacement medication. The step width differs from previous findings of transitional movements (Morris et al 2001) in that the PD group displayed a wider step during the first step of gait termination. Despite being equally stable to the control group in the medial-lateral plane, the PD participants were less stable throughout both steps of GT in the anterior-posterior plane. The increased step width during GT may have been an attempt by the PD group to enhance their stability as they stopped.

Both groups created a larger, lateral margin of stability at the start of GT when the stop was planned compared to when it was a cued stop, but were equally stable during the final step of the stop in both conditions. The increased stability margin may have been a 
deliberate, stability-enhancing action that took more time to execute than the cued GT trials allowed for in that first step. The PD group, therefore, was able to take advantage of the opportunity for motor planning in the same manner the control group did. Also, both the control and PD groups were able to slow forward progression and stop within a similar time which suggests that Parkinson's disease does not affect the ability to stop within a normal amount of time.

Both planned and cued non-slippery stops performed in this study required the participant to initiate the GT command. Planned GT allowed the motor program to be generated and carried out over multiple steps. Cued GT required the motor program to change from continuous gait to a stop within two steps. The PD group was able to stop safely

and effectively in both conditions. Their increased step width may have been a strategy used to compensate for the diminished stability in the plane of progression but overall, the PD group was able to perform both planned and cued GT similar to age-matched controls. The timing of the gait termination program was similar between groups despite the decreased walking velocity of the PD group one step before the force plates. The stability of the timing of the movements suggests a common strategy used for both planned and cued gait termination (Bishop et al 2003).

\subsubsection{Non-slippery versus slippery stops}

The approach velocity at the time the signal to stop was given is similar for both conditions suggesting that the participants did not anticipate the stop or the slip trial. The 
increased step length after the slip is due to the forward movement of the foot on the force plate and does not indicate any behvioural modification resulting from the slip.

The ability of both groups to slow their forward progression faster in the slippery stop trials than the non-slippery stop trials suggests that the CNS of both control and PD participants recognized that the slip caused unwanted movement forward so they compensated quickly to try and stop the excess movement. The participants were not able to completely stop within the same time frame as a non-slippery stop suggesting that, although they recognized and began to slow forward progression faster, the slip required more time to stabilize and completely stop on the moving surface.

Past research has suggested that dopamine replacement therapy does not increase the ability of someone with PD to effectively handle external perturbations (Carpenter et al 2004; Frank et al 2000; Horak et al 1996). The major difference between the participants with PD and the age-matched controls were the step width and dynamic stability in the plane of progression. The instability may be a consequence of the increased mobility produced by the medication and the increased step width may be an attempt to correct for this instability during GT.

In summary, the participants with PD were able to accurately stop in all conditions. Their strategy was slower and safer than the age-matched controls. When there is opportunity to plan the stop multiple steps in advance, both groups increased their M-L dynamic stability during the first step of GT. The cueing did not have any other effect on the participants in that they were able to perform both the planned and the cued GT within the same time frames 
of each other. The unexpected slip during GT did, however, change the behaviours of both groups by slowing their forward progression earlier than in a non-slippery. The generalized slip response seen in previous studies examining slips during gait (e.g., Marigold and Patla 2002 (low friction apparatus), Tang and Woollacott 1999 (unexpected anterior platform translation) and gait termination (Oates et al 2005 (low friction apparatus)) was seen in both the control and PD group which suggests the CNS develops a patterned response to a slippery perturbation that is not affected by age or PD. Parkinson's disease did not seem to affect the ability to integrate a balance correcting response with a voluntary movement such as gait termination but did cause instability throughout gait termination in the plane of progression. Future research should question how quickly these groups are able to adapt to stopping on a slippery surface and if Parkinson's disease affects the ability to modify their behaviours. 


\subsection{REFERENCES}

Abbruzzese G, and Berardelli A. (2003). Research Review: Sensorimotor integration in movement disorders. Movement Disorders, 18(3): 231-240.

Bishop B, Brunt D, Kukulka C, Tillman MD, and Pathare N. (2003). Braking impulse and muscle activation during unplanned gait termination in human subjects with Parkinsonism. Neurosci Letters, 348: 89-92.

Bishop M, Brunt D, and Marjama-Lyons J. (2006). Do people with Parkinson's disease change strategy during unplanned gait termination? Neuroscience Letters, 397: 240-244.

Bloem BR and Bhatia KP. (2004). Gait and balance in basal ganglia disorders. In Clinical Disorders of Balance, Posture and Gait ( $2^{\text {nd }}$ Edition). Eds. Bronstein AM, Brandt T, Woollacott MH, and Nutt JG: 173-206.

Carpenter MG, Allum JHJ, Honegger F, Adkin AL, and Bloem BR. (2004). Postural abnormalities to multidirectional stance perturbations in Parkinson's disease. J Neurol Neurosurg Psychiatry, 75: 1245-1254.

Dimitrova D, Horak FB, and Nutt JG. (2004). Postural muscle responses to multidirectional translations in patients with Parkinson's disease. J Neurophysiol, 91: 489-501.

Frank JS, Horak FB, and Nutt J. (2000). Centrally initiated postural adjustments in Parkinsonian patients on and off levodopa. J Neurophysiol, 84: 2440-2448.

Hase K, and Stein RB. (1998). Analysis of rapid stopping during human walking. J Neurophysiol, 80: 255-261.

Hof AL, Gasendam MGJ, and Sinke WE. (2005). The condition for dynamic stability. J Biomechanics, 38: 1-8.

Horak FB, Frank J, and Nutt J. (1996). Effects of dopamine on postural control in Parkinsonian subjects: Scaling, set, and tone. J Neurophysiol, 75(6): 2380-2396.

Jaeger R, and Vanitchatchavan P. (1992). Ground reaction forces during termination of human gait. J Biomech, 25 (10): 1233-1236.

Marigold DS, and Patla AE. (2002). Strategies for dynamic stability during locomotion on a slippery surface: effects of prior experience with and knowledge about surface characteristics. J Neurophysiol, 88: 339-353.

Morris ME. (2006). Locomotor training in people with Parkinson disease. Physical Therapy, 86(10): 14261435.

Morris ME, Huxham F, McGinley J, Dodd K, and Iansek R. (2001). Review: The biomechanics and motor control of gait in Parkinson disease. Clinical Biomechanics, 16: 459-470.

Nieuwboer A, Dom R, De Weerdt W, Desloovere K, Janssens L, and Vangheluwe S. (2004). Electromyographic profiles of gait prior to onset of freezing episodes in patients with Parkinson's disease. Brain, 127: 1650-1660.

Oates AR, Patla AE, Frank JS, and Greig MA. (2005). Control of dynamic stability during gait termination on a slippery surface. J Neurophysiol, 93:64-70.

Tang PF, and Woollacott MH. (1999). Phase-dependent modulation of proximal and distal postural responses to slips in young and older adults. J Gerontol: MED SCI, 54A(2): M89-M102. 
CHAPTER 5: ADAPTATION TO GAIT TERMINATION ON A SLIPPERY SURFACE WITH PARKINSON'S DISEASE 


\subsection{INTRODUCTION}

Stopping on a slippery surface requires a reactive balance-correcting response to be integrated into a voluntary, gait termination program. Knowledge of and experience with the slippery surface allows the use of feed forward control to increase stability and stop more safely and efficiently (Bhatt et al 2006; Marigold and Patla 2002). The behavioural adaptations when encountering a slippery surface include a decreased step length when approaching the surface (Bhatt et al 2006; Brady et al 2000; Cham and Redfern 2002; Lockhart et al 2006; Moyer et al 2006), flattened foot (Brady et al 2000; Cham and Redfern 2002; Heiden et al 2006; Marigold and Patla 2002; Moyer et al 2006), diminished arm raise (Marigold and Patla 2002; Marigold et al 2003; Tang and Woollacott 1998), decreased muscular effort (Marigold and Patla 2002) and lengthened subsequent step (Marigold et al 2003; Myung and Smith 1997; Tang and Woollacott 1998).

Parkinson's disease (PD), caused by decreased dopaminergic activity from the basal ganglia, is a disease causing movement and balance difficulties (Abbruzzese and Berardelli 2003; Frank et al 2000; Horak et al 1996; Morris 2006). Parkinson's disease disrupts the ability to adapt to new environments, learn a new skill and can limit the ability to quickly change motor programs (Bishop et al 2006; Morris 2006). PD also affects sensorimotor integration and can interfere with the integration of a reactive and proactive motor program (Abbruzzese and Berardelli 2003). As PD progresses, gait termination can become more difficult as people with PD may exhibit festination when attempting to stop (Bloem and Bhatia 2004; Morris 2006). Traditional dopaminergic replacement therapy seems to have 
little effect on the ability to handle external balance perturbations (Carpenter et al 2004; Frank et al 2000; Horak et al 1996; Morris 2006; Morris et al 2001). Developing a proactive gait termination to minimize the reliance on a reactive strategy may be very difficult for a person with PD even with optimal medication. External cues, such as a light source or planned stop location may help someone with PD rely more on the frontal area of the brain to successfully complete a stop within similar time-lines as age-matched controls (Morris 2006; Morris et al 2001).

This study investigates the ability of someone with PD to integrate a reactive, balance-correcting response into a proactive, voluntary gait termination program. The effect of experience with and knowledge of the slippery surface as well as the abilities to plan a movement strategy within one step and over multiple steps is examined in participants with PD and age-matched controls. The differences in behaviours between the participants with PD and the control group will show how the basal ganglia is involved in the integration and planning elements of gait termination on a slippery surface. We hypothesize that PD will affect the ability to develop a stable, proactive response to stopping on the slippery surface in that the PD group will take longer to show adaptations and will be less stable than the control group. 


\subsection{METHODS}

\subsubsection{Protocol}

Nine participants with idiopathic PD (age $66.3+/-7.3$ years SD) and ten age-matched controls (age $65.4+/-7.3$ years SD) participated in this study (see table 1 for PD participant characteristics). All PD participants were on their usual medication during testing. Before data collection, a modified UPDRS examination was administered to examine the motor capabilities of individuals with PD. The severity of Parkinsonism, as measured by the Hoehn and Yahr scale ranged from 1 to 3 . All participants were able to walk independently and reported that they were free of orthopaedic, psychological, or other neurological disorders which could affect their ability to perform the tasks. A safety harness was worn throughout data collection. All participants provided written, informed consent for protocols approved by the Institutional Review Board for OHSU. Approval for this protocol was also obtained through the University of Waterloo's Office of Research Ethics.

All participants experienced four types of trials: 1) Walk-through trials for baseline

Table 5.1: Age, Motor UPDRS score and Hoehn and Yahr stage for PD participants.

\begin{tabular}{cccc}
\hline ID & Age & UPDRS & H \& Y \\
\hline \hline 1 & 71 & 31.5 & 2.5 \\
2 & 68 & 36 & 2 \\
3 & 67 & 40 & 3 \\
4 & 62 & 42.5 & 2 \\
5 & 51 & 36.5 & 2 \\
6 & 78 & 44 & 3 \\
7 & 63 & 7 & 1 \\
8 & 73 & 29 & 2 \\
9 & 62 & 24 & 2 \\
& & &
\end{tabular}


gait information, 2) one unexpected cued slippery stop to measure the effect of PD on the first slip response during gait termination (previously reported in Chapter 4), 3) five planned slippery stops to show the effect of PD on the ability to plan a slippery stop over multiple steps with repeated exposures, and 4) five cued slippery stops to show the effect of PD on adaptation to stopping on a slippery surface within one step with repeated exposures. The participants were asked to walk to the end of the room towards a set of lights. The lights were round, approximately $10 \mathrm{~cm}$ in diameter, resembled the red brake lights on a small motor vehicle and were located at eye level at the far end of the room. If the lights did not illuminate then they were to continue walking to the end of the room. If the lights illuminated then they were to stop with both feet on the force plates. The lights at the end of the room were controlled by placing an infrared light beam positioned at left foot contact (one step) before reaching the force plates. When the light beam was interrupted during the cued stop trials, the lights illuminated. The participant received the signal to stop on the left limb (here after referred to as the trail limb), stepped on a force plate with the right limb (here after referred to as the lead limb) and then finished gait termination with the trail limb on a force plate next to the lead foot. Participants were not told to try to step on the force plates but to walk normally towards the end of the room. Their starting location was manipulated so that they would step on the right force plate with their lead foot.

After a series of cued non-slippery stop trials (see chapter 4), participants stepped with their lead limb on to the force plate which moved forward quickly and unexpectedly mimicking a slippery surface (unexpected slippery stop). The plate movement was 
controlled using a force-integration function where the plate continued to accelerate until it reached its maximum displacement (an average of $14.7 \mathrm{~cm}$ forward at $0.47 \mathrm{~m} / \mathrm{s}$ ). This perturbation is similar in displacement but slower in slip velocity to previous investigations of stopping on a slippery surface (Oates et al 2005). After the first unexpected cued slippery stop trial, participants then performed a series of five planned slippery stop trials in a row. For the planned slippery stop trials, the lights at the end of the room were illuminated the entire trial. Participants knew at the start of the trial that they were going to stop on the force plates and that the right force plate would slip underneath their lead foot. The participants then performed a series of five cued-slippery stop trials where they were asked to stop only if the lights illuminated. The cued slippery stop trials were randomly placed among 15 walkthrough trials and participants were told that if they were asked to stop, the force plate would move forward under their lead limb. The planned slippery stops were always performed after the unexpected, cued slippery stop and before the cued slippery stop trials. This order was chosen to allow practice with knowledge of the stop and the slippery surface (planned slippery stops, the easier task) before requiring a stop within one step on a slippery surface (cued slippery stops, the more difficult task) as it was hypothesized that the PD group would be less stable and this grouping of trials would decrease the chance of a fall.

\subsubsection{Measurement}

Kinetic data were captured from the custom-made force plates using a QNX data collection system ( sampling rate $=480 \mathrm{~Hz}$ ) and used for identifying foot contact on the moving force plate. A high-resolution Motion Analysis System (Santa Rosa, CA) with 7- 
video cameras, sampling at $60 \mathrm{~Hz}$, provided 3-dimensional spatial coordinate information about body segment displacements. Markers were placed on multiple anatomical landmarks bilaterally including the ear, acromion process, olecranon, styloid process, anterior superior iliac crest, greater trochanter, lateral femoral condyle, lateral malleolus, heel and fifth metatarsal, and on the xyphoid process.

\subsubsection{Analysis}

Step length and width were calculated from the heel markers of both feet. Step length was defined as the anterior-posterior (A-P) distance from the trail limb heel marker to the lead limb heel marker in subsequent steps. Step width was defined as the absolute mediallateral (M-L) distance between heel markers in subsequent steps. Qualitative assessment of the video data showed that the arm raise was produced with a fairly straight arm and, therefore, the maximum height of both arms was calculated as the maximum height of the marker on the radial styloid within the first $500 \mathrm{~ms}$ after heel contact on the rollers. Values were normalized to the slippery stop trial to account for different arm lengths and body heights.

A 12-segment centre of mass (COM) model was calculated using a custom-designed MATLAB program (Mathworks, Natick, MA) with marker data low-pass filtered at 6Hz. $\mathrm{COM}$ velocity was calculated by differentiating the COM displacement. The peak downward COM displacement was determined after heel contact on the slippery surface. Time to slow was defined as the time when the COM velocity in the anterior-posterior plane fell below the average minus two standard deviations of the average forward velocity of the walk-through 
trials. The time to stop was defined as the time when forward velocity fell within the average plus two standard deviations of the average velocity (over 500ms) one second after the right heel contact on the first force plate of previously performed planned non-slippery stop trials (see chapter four). The time to slow represents the beginning of gait termination; the time to slow represents the completion of gait termination.

A margin of dynamic stability was calculated using the method developed by Hof et al (2005) which calculates an extrapolated COM position (xCOM) using the equation: $\mathrm{xCOM}$ $=\mathrm{COM}$ position $+\mathrm{COM}$ velocity $/ \sqrt{ }(\mathrm{g} / \mathrm{l})$, where $\mathrm{g}=$ acceleration due to gravity and $\mathrm{l}=$ vertical height of the COM. The position of the $\mathrm{xCOM}$ was compared to the position of the marker on the fifth metatarsal on the foot that was stepping (i.e., to the right foot during the first step of GT and to the left foot during the second step of GT) at the time of foot contact. This analysis incorporates both the relative position of the sagittal COM with respect to the foot and the velocity of the COM. The magnitude of the difference in the horizontal planes is the dynamic margin of stability.

Some participants took one or more extra steps (i.e., beyond the first step with the lead limb and the second step with the trail limb) during the slippery stop trials. The number of extra steps was counted and analyzed using a Chi-square analysis $(\alpha=.05)$ to identify any group or trial effects. Discrete data were entered into a group (two levels) by trial (11 levels) one- way repeated measures ANOVA to examine any effects of group or experience over the slip trials $(\alpha=0.05)$. The one-way ANOVA was chosen due to the blocked nature of the trials; the cued slippery stops always followed the planned slippery stops. Planned 
comparisons were made using Tukey's studentized range (HSD) post-hoc tests: To determine when a change in behaviour occurred within conditions, the unexpected slippery stop was compared to the trials of the planned slippery stops and also the trials of the cued slippery stops, separately. To determine how the condition (planned versus cued) affects behaviour, the first cued slippery stop was compared to the final planned slippery stop. Any interaction effects between groups and trials were analyzed in a one-way repeated measure ANOVA with each group separately (i.e., one level for group and 11 levels for condition). All post-hoc differences are reported with a significance level of $\alpha=0.05$. Reported trends are based on qualitative observations and do not imply statistical significance.

\subsection{RESULTS}

\subsubsection{Group effects}

Participants with PD walked slower than the age-matched controls ( $\mathrm{F}=14.18$, $\mathrm{p}=.0015$ ) with mean velocities of $1.0 \mathrm{~m} / \mathrm{s}$ and $1.33 \mathrm{~m} / \mathrm{s}$ for the $\mathrm{PD}$ and control groups, respectively. There were no differences in the time to slow or stop, step parameters, arm raises, or peak downward COM displacement. The PD group had a significantly smaller M-L margin of dynamic stability than controls $(\mathrm{PD}=10.41+/-0.21(\mathrm{SE}), \mathrm{CT}=11.98+/-0.20$ (SE)) during the first step with the lead limb onto the force plate $(\mathrm{F}=5.19, \mathrm{p}=.0359)$ when averaged across trials. The only significant interaction in the analysis was the A-P margin of dynamic stability during the second step of GT $(\mathrm{F}=1.98, \mathrm{p}=.0389)$. There was a trial effect within the PD group $(\mathrm{F}=2.281, \mathrm{p}=.022)$ but there were no significant differences that fell 


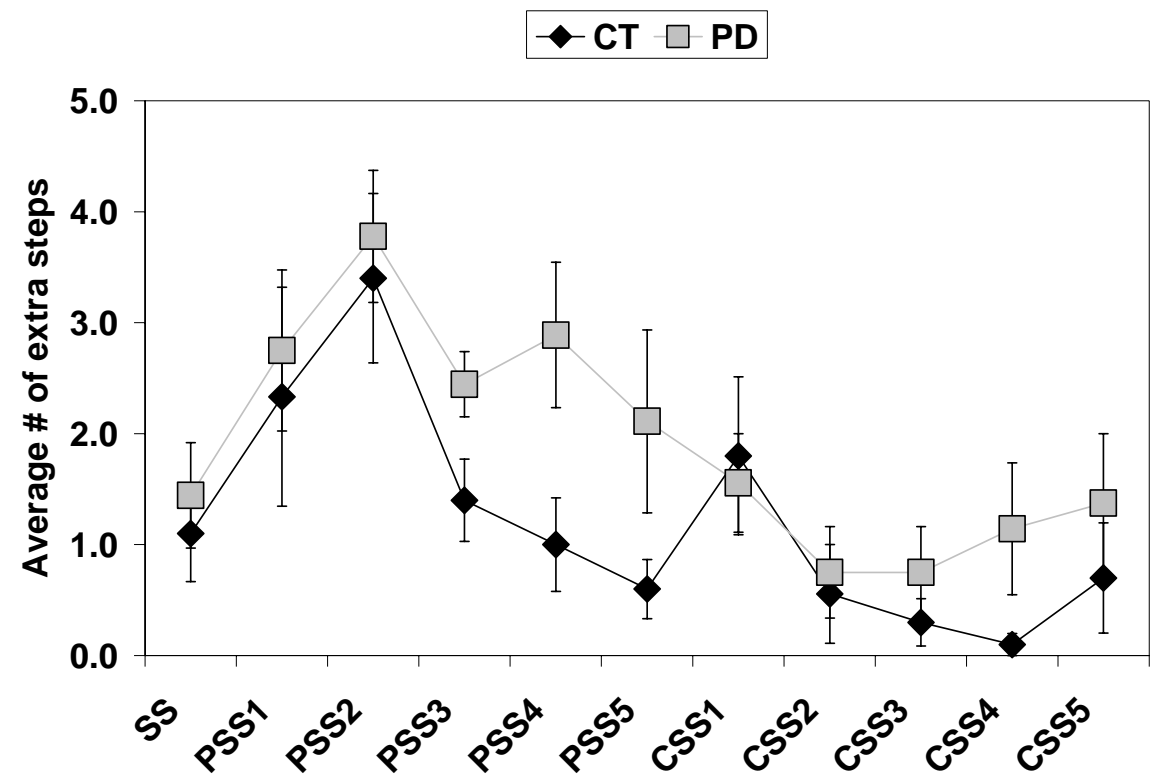

Figure 5.1: Average number of extra steps taken (+/- SE). (SS = unexpected cued slippery stop, PSS = planned slippery stop, CSS = cued slippery stop).

within the planned comparisons. The control group showed no significant trial effect during the second step of GT for the margin of dynamic stability in the plane of progression. Chisquare analysis revealed that participants in the PD group took more extra steps than the control group $(p=.005)$ (figure 5.1). Qualitative observation of figure 5.1 and the differences between the PD and control groups over the subsequent slippery stops suggests that the PD group showed less adaptation (i.e., smaller decreases with repeated experiences) than the control group.

\subsubsection{Trial effects}

There was a significant trial effect in the walking velocity $(F=14.18, p=.0015)$. The second planned slippery stop was slower than the unexpected slippery stop but the remaining planned slippery stops were similar to the unexpected slippery stop. The velocities for the 
cued slippery stops were similar to the unexpected slippery stop across all cued, slippery stop trials. There was a significant trial effect in the time to slow $(\mathrm{F}=2.51, \mathrm{p}=.0079)$ and in the time to stop $(\mathrm{F}=6.06, \mathrm{p}<.0001)$. Both groups slowed their forward progression later in the second planned slippery stop compared to the unexpected slippery stop. There was no difference in the time to slow between the unexpected slippery stop and the cued slippery stops. The time to stop in the first and second planned slippery stops was later than the unexpected slippery stop. There was no difference in the time to stop between the unexpected slippery stop and the cued slippery stops.

Step length showed a significant trial effect during the first step with the lead limb onto the force plate $(\mathrm{F}=6.44, \mathrm{p}<.0001)$ and during the second step with the trail limb of gait termination $(\mathrm{F}=9.79, \mathrm{p}<.0001)$ (figure 2). During the first step of gait termination, step length decreased from the unexpected slippery stop by the third planned slippery stop. The step length values for all of the cued slippery stops were shorter than the unexpected slippery stop. The step with the trail limb increased immediately after the unexpected slippery stop trial and was longer for all of the following planned and cued slippery stop trials.

There was a significant trial effect in step width during the first step with the lead limb onto the force plate $(\mathrm{F}=2.77, \mathrm{p}=.0034)$ and during the second step with the trail limb $(\mathrm{F}=4.97, \mathrm{p}<.0001)$ (figure 5.3). During the first step, the step width was wider for the third planned slippery stop trial compared to the unexpected slippery. Step width increased after the unexpected slippery stop during the subsequent step with the trail limb throughout all of the planned slippery stop trials and the final three cued slippery stop trials. 
The margin of dynamic stability showed a significant trial effect during the first step in the A-P $(\mathrm{F}=6.68, \mathrm{p}<.0001)$ and $\mathrm{M}-\mathrm{L}$ planes $(\mathrm{F}=2.83, \mathrm{p}=.0029)$, and a significant interaction during the second step in the A-P plane (previously discussed in the group effects). The margin of dynamic stability in the plane of progression was lower in the final four planned slippery stops and all of the cued slippery stops when compared to the unexpected slippery stop trial during the first step onto the force plate. There were no differences between trials for the second step with the trail limb in the margin of A-P

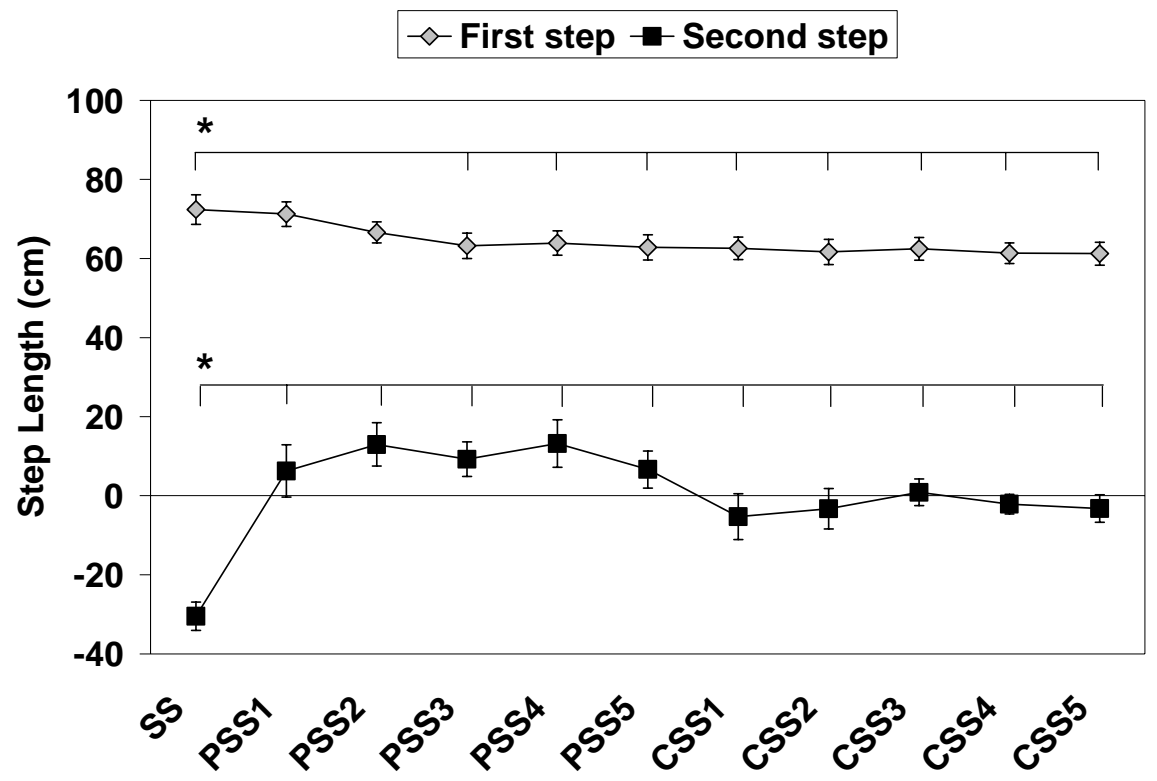

Figure 5.2: Step length during the steps onto the force plate (lead limb) and subsequent, second and final step of gait termination (trail limb) $(+/$ - SE). * indicates a significant difference between that condition and the others indicated with a vertical line. ( $\mathrm{SS}=$ unexpected cued slippery stop, PSS = planned slippery stop, CSS = cued slippery stop).

dynamic stability. The margin of dynamic stability in the M-L plane during the first step onto the force plate was smaller for the unexpected slippery stop compared to the third planned slippery stop. There was no difference from the unexpected slippery stop in any of the subsequent trials during the second step in the margin of M-L dynamic stability. 


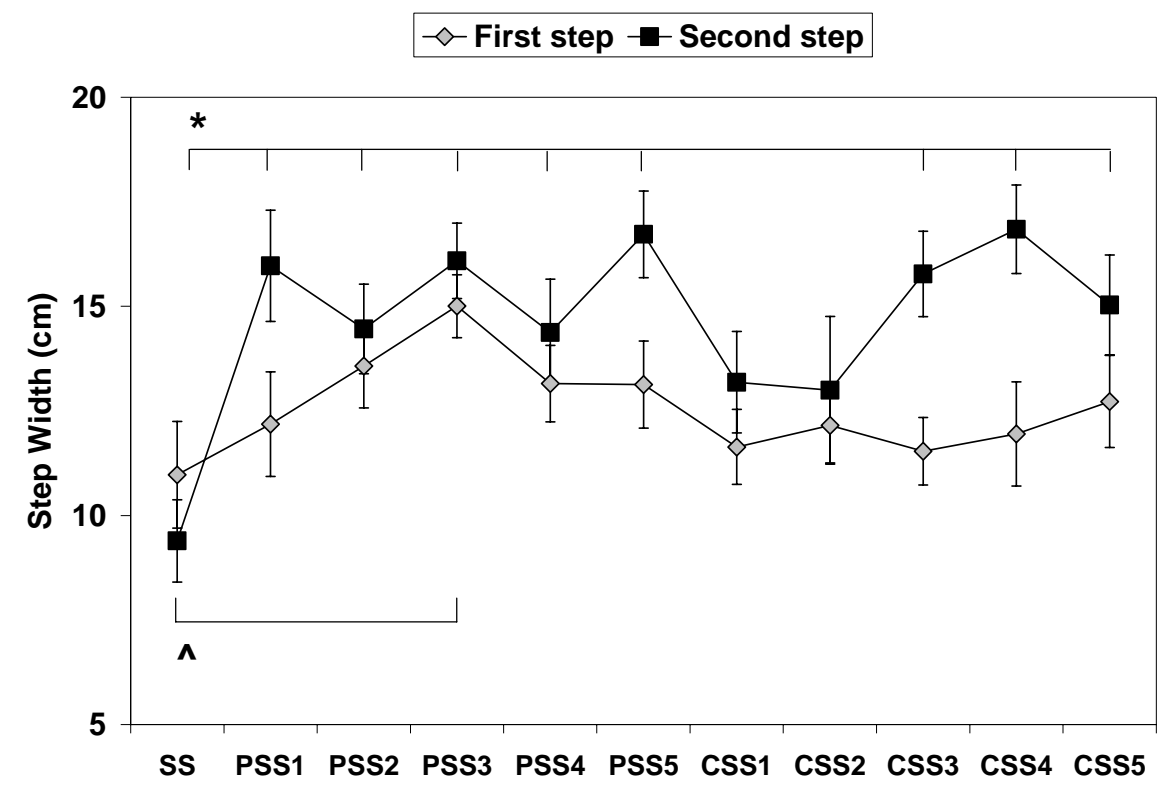

Figure 5.3: Step width during the first step onto the force plate (lead limb) and the

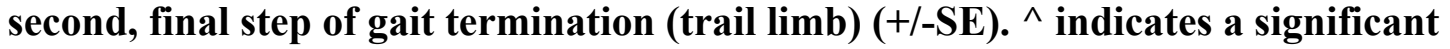
difference in the first step and * indicates a significant difference in the second step between that condition and the others indicated by the horizontal lines. (SS = unexpected cued slippery stop, PSS = planned slippery stop, CSS = cued slippery stop).

There was no trial effect seen in the peak downward COM movement $(\mathrm{F}=1.03$, $\mathrm{p}=.4195$ ) suggesting that participants lowered their COM equally during all slip trials. Both the right $(\mathrm{F}=2.85, \mathrm{p}=.0027)$ and left $(\mathrm{F}=7.33, \mathrm{p}<.0001)$ arms, however, showed a significant trial effect in the height of arm movement used during the slips. The right arm showed no differences within our planned comparisons but the left arm was raised higher during the unexpected slippery stop compared to the final planned slippery stop, and the final three cued slippery stop trials (figure 5.4). 


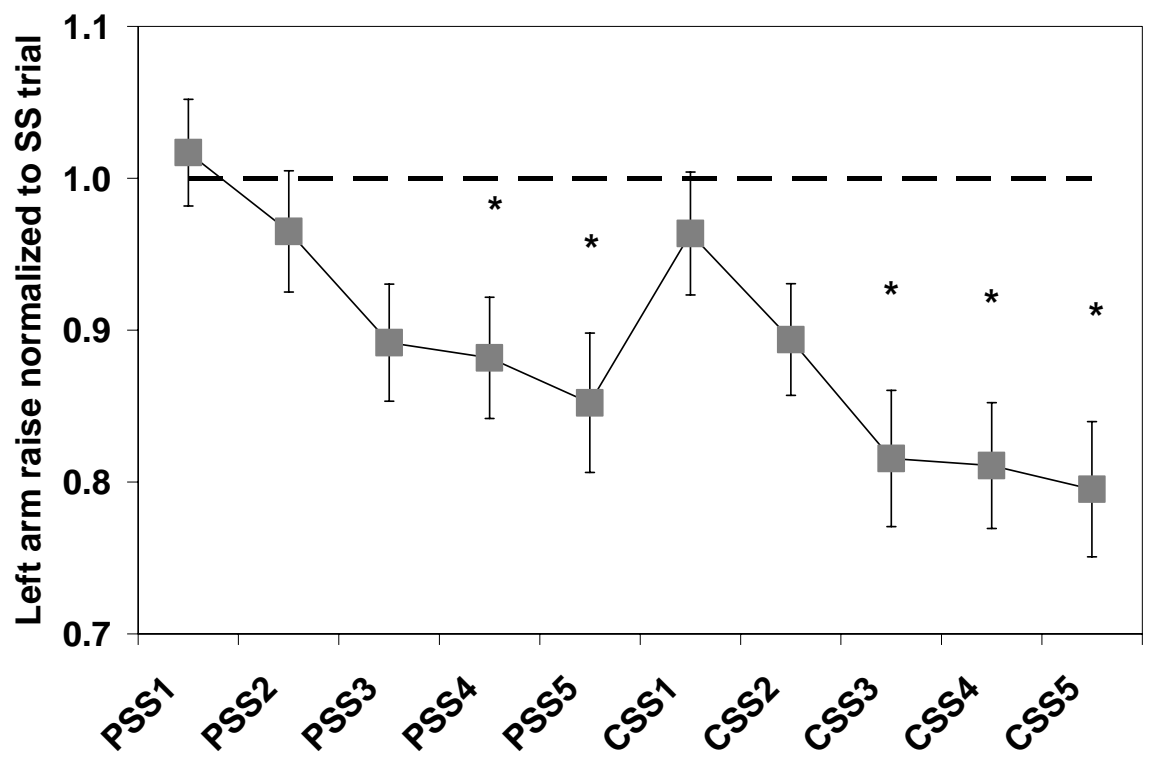

Figure 5.4: Average left arm raise for both groups normalized to unexpected cued slippery stop trial $(+/-\mathrm{SE}) .($ Value $=$ trial/SS trial). $*$ indicates a significant difference between that trial and the unexpected cued slippery stop trial (dashed line). (PSS = planned slippery stop, CSS = cued slippery stop).

The chi-square analysis revealed a significant trial effect for the number of steps taken beyond normal gait termination but does not allow for post-hoc comparison. The plot, however, suggests that participants took more extra steps during the planned slippery stops than the unexpected slippery stop and the cued slippery stop trial (based on trend) (figure $5.1)$.

There seemed to be some trend in the data showing a regression towards the behaviour seen in the unexpected slippery stop when conditions change from planned to cued slippery stops. These trends were not significant suggesting that the type of knowledge (in advance during the planned and within one step during the cued slippery stops) does not affect the adaptation to the slippery surface. 


\subsection{DISCUSSION}

Both the control and the PD group showed adaptations to their behaviour while stopping on a slippery surface. The PD group showed very similar behaviour to the control group suggesting that PD did not affect the ability to integrate a balance-correcting response into a proactive, voluntary gait termination program. The similarity also suggests that the basal ganglia are not involved in the integration and planning elements of gait termination, or in adapting these movements, on a slippery surface. Adaptations included a shorter step onto the force plate, a longer and wider subsequent step, and a diminished arm raise. These adaptive changes usually occurred within three trials of the unexpected slippery stop.

\subsubsection{Integration of reactive and voluntary postural adjustments during gait termination are preserved in PD}

Parkinson's disease seemed to have little effect on the ability to integrate a balancecorrecting response into a voluntary gait termination program or the ability to develop an adaptive strategy to effectively deal with the slippery stops. The PD group walked slower and was less stable than the control group as they took more extra steps and had a smaller margin of M-L dynamic stability during the first step onto the force plate. The interaction effect shown in the A-P margin of dynamic stability during the second step showed no difference in the planned comparisons and is, therefore, not a significant behavioural change. The PD group did, however, seem to show a slower adaptation in the number of extra steps than controls which may reflect instability during the slippery stops which required more extra steps to stop safely. 
People with PD are able to use external cues, visual or sensory, to initiate motor commands from cerebral sources other than the basal ganglia (Abbruzzese and Berardelli 2003; Morris 2006; Morris et al 2001). The PD group may have used the visual cue of the lights during the cued, slippery stop trials and the feedback from the lead limb during the slip to help initiate the gait termination and slip-response programs, respectively. Because PD disrupts the ability to use central set to adapt voluntary movements (Morris 2006) and external reactions caused by changes in the base of support (Horak et al 1996) the external cues may have initiated the motor programs from regions within the CNS other than the basal ganglia (Morris 2006; Morris et al 2001).

The participants with PD in this study were able to show similar adaptations to the slippery surface as age-matched controls in both planned and cued slippery stop trials. Previous research suggests that one set of motor commands is used in both planned and unplanned (cued) gait termination in persons with Parkinson's disease (Bishop et al 2006). Since there was no significant difference between the last planned slippery stop and the first cued slippery stop, a single strategy may have been developed and used for both planned and cued slippery stops.

\subsubsection{Adaptations to gait termination were observed for planned and cued slippery stops}

All participants showed behavioural adaptations to the slippery surface encountered during gait termination. Table 2 is a summary of when the independent variables changed significantly from the first unexpected slippery stop trial. Only those variables which showed 
a persistent change are considered an adaptive change. The decreased velocity in the second planned stop is likely the cause for the earlier time to slow in the second planned slippery stop. After the unexpected slip, it takes longer to stop in the following two planned slippery stops and then behaviour becomes similar to the unexpected slippery stop. This change in the time to stop is not likely an adaptation as it did not persist over the following slippery stop trials but rather was an adjustment to the slippery stop while a movement strategy was being developed. The lack of difference between the cued slippery stops and the unexpected slippery stops in the walking velocity, time to slow and time to stop suggests that none of these measures affects the adaptation to stopping on a slippery surface.

Changes in behaviour during the step onto the force plate (pre-slip) show proactive adaptations to the slippery surface where changes in behaviour during the second step (postslip) show adaptations to the reactive component of the motor program for GT on a slippery surface. Grouping measurements in one dimension show how stability is controlled in that plane (step length and margin of A-P dynamic stability in the plane of progression and step width and margin of M-L dynamic stability in the lateral plane).

Proactive adaptations in the plane of progression took longer to adapt (maintain a difference from the unexpected slip) during the planned slippery stops than the cued slippery stops. Step length was decreased by the third planned slippery stop but the decrease in the cued slippery stop was seen in the first trial. The margin of A-P dynamic stability took two trials to show an adaptation in the planned slippery stops and only one in the cued slippery stop trials. One proactive adjustment was seen in the lateral plane at the third planned 
slippery stop: The step width increased thereby increasing the base of support. The increased base of support is also reflected in the increased margin of M-L dynamic stability during the third planned stop when compared to the first unexpected slip. Because these increases only occur in one trial, the effect cannot be interpreted as an adaptation.

Table 5.2: Summary table for the dependent measures showing when an adaptation occurred. The comparisons were with the unexpected slippery stop (SS). Information in parentheses indicates when one trial was different from the SS trial but the change did not persist across trials in that condition. No difference from the SS trial is indicated by n.d.

\begin{tabular}{lcc}
\hline Dependent Measure & Planned slippery stops & Cued slippery stops \\
\hline \hline Walking velocity & (PSS2 <SS) & n.d. \\
Time to slow & (PSS2 <SS) & n.d. \\
Time to stop & (PSS1 and PSS2 $>$ SS) & n.d. \\
Step length: Lead limb & PSS3 & n.d. \\
Step length: Trail limb & PSS1 & CSS1 \\
Step width: Lead limb & (PSS3 $>$ SS) & n.d. \\
Step width: Trail limb & PSS1 & CSS3 \\
Margin of dynamic stability: & & \\
A-P lead limb step & PSS2 & CSS1 \\
A-P trail limb step & n.d. & n.d. \\
M-L lead limb step & (PSS3 $>$ SS) & n.d. \\
M-L trail limb step & n.d. & n.d. \\
Peak downward COM movement & n.d. & n.d. \\
Left arm raise & PSS4 & CSS3 \\
Right arm raise & n.d. & n.d.
\end{tabular}

Reactive changes in the plane of progression occurred only in the step length and not in the margin of dynamic stability. The adaptations to step length occurred in the first trial for both planned and cued slippery stops. The quickness of the adaptation suggests the reactive component is more effective at adapting to the slippery surface than the proactive component and, therefore, adjusts earlier. Adaptations in the lateral plane were seen only in the step width and occurred later in the cued slippery stops than the planned slippery stops. The 
delayed adaptation in the cued slippery stops suggests that knowledge of when the stop will occur can be used to develop a slip response strategy in the M-L plane.

The arm raises can be considered as part of the reactive component to the slip response strategy as they occur after the slip. The arms took longer to show an adaptation than any other behaviour that did show an adaptation and may, therefore, be an integral part of the balance correcting response to gait termination on a slippery surface.

The lack of difference between the final planned and first cued slippery stop trials suggests that there is no difference in generating a slippery stop response over multiple steps compared to within one step. The similarities may also be due to the repeated experiences (one unexpected and six planned slippery stops) leading up to the first cued slippery stop. Because the cued slippery stop trials always occurred after the planned slippery stop trials there is no way to completely remove the effect of experience and examine any difference between planning and cueing GT on a slippery surface with the protocol used in this study.

The age of participants and the external validity of the imposed slip must be considered when discussing the results of this study. Both groups had a mean age over 65 years and could be considered older adults. Previous research into the adaptations to slips during locomotion reveal differences in the behaviour of older adults when compared to younger adults performing the same task. These studies have shown that older adults are more cautious and may change their gait earlier (Lockhart et al 2006) when approaching and traveling over a slippery surface by walking slower and with shorter steps (Lockhart et al 2005 and 2006; Moyer et al 2006; Tang and Woollacott 1998). Age seems to slow the 
reaction time and diminish the power of the slip response (Chambers and Cham 2006; Lockhart et al 2005; Tang and Woollacott 1998) which may increase the frequency of taking an extra step (Tirosh and Sparrow 2003). The adaptive changes to stopping on a slippery surface are similar to young, healthy adults, but seem to require at least one more experience with the slippery surface to show adaptive changes (chapter 3). Age does not affect the ability to adapt to a slippery surface and develop a slip-response strategy as seen in this study and others (e.g. Lockhart et al 2006; Moyer et al 2006; Tang and Woollacott 1998) but may limit the flexibility of this slip-response strategy to adapt to different slipping situations (Tang and Woollacott 1999).

The perturbation used in this study was a moving force plate programmed to mimic the feeling of a slippery surface. The external validity of this slip is limited in that research shows (Troy and Grabiner 2006) that true slips (such as those on an ice surface) are more destabilizing than platform slips. The platform slips also offer an end-point to the stop which may mechanically aid in the slip-recover process where real-world slips may not have a boundary close enough to help recovery. The platform will also sustain larger lateral loads than ice which can be manipulated to aid in the slip recovery. Additionally, the feedback from a moving platform or a surface causing the foot to move forward creates a different type of feedback from that received when the foot slides over a slippery surface. Perry et al (2000) found that afferent information from the cutaneous receptors in the foot detect the boundaries of the BOS are important in controlling the responses to balance perturbations. For this protocol, the cutaneous afferents did not receive the same information while sliding with the 
force plate that they would have received had the foot slid over the floor. The addition of cutaneous afference may have aided in the slip recovery and enabled a quicker adaptation to the slip.

In summary, the PD group walked slower and was less stable than the control group while stopping on a slippery surface. Parkinson's disease did not seem to affect the ability to integrate a reactive response into a voluntary movement or to adapt the movement strategy to repeated experiences on the slippery surface. Adaptations to the slippery surface included a shortened step onto the slippery surface, an increased subsequent step length, increased step width and a diminished arm response. Proactive adaptations occur within three experiences with the slippery surface. During planned slippery stops, reactive adaptations occur within two trials in the plane of progressions and within one trial in the lateral plane. During cued slippery stops, reactive adaptations occur within one trial in the plane of progression and within three trials in the lateral plane. The arm response persists longer than any other behaviour that shows an adaptation and, therefore, seems to be important in maintaining stability while stopping on a slippery surface. 


\subsection{REFERENCES}

Abbruzzese G, and Berardelli A. (2003). Research Review: Sensorimotor integration in movement disorders. Movement Disorders, 18(3): 231-240.

Bhatt T, Wang E, and Pai Y-C. (2006). Retention of adaptive control over varying intervals: Prevention of slipinduced backward balance loss during gait. J Neurophysiol, 95: 2913-2922.

Bishop B, Brunt D, Kukulka C, Tillman MD, and Pathare N. (2003). Braking impulse and muscle activation during unplanned gait termination in human subjects with Parkinsonism. Neurosci Letters, 348: 89-92.

Bishop M, Brunt D, and Marjama-Lyons J. (2006). Do people with Parkinson's disease change strategy during unplanned gait termination? Neurosci Letters, 397: 240-244.

Bloem BR and Bhatia KP. (2004). Gait and balance in basal ganglia disorders. In Clinical Disorders of Balance, Posture and Gait ( $2^{\text {nd }}$ Edition). Eds. Bronstein AM, Brandt T, Woollacott MH, and Nutt JG: 173-206.

Brady RA, Pavol MJ, Owings TM, and Grabiner MD. (2000). Foot displacement but not velocity predicts the outcome of a slip induced in young subjects while walking. J Biomech, 33: 803-808.

Carpenter MG, Allum JHJ, Honegger F, Adkin AL, and Bloem BR. (2004). Postural abnormalities to multidirectional stance perturbations in Parkinson's disease. J Neurol Neurosurg Psychiatry, 75: 1245-1254.

Chambers AJ, and Cham R. (2006). Slip-related muscle activation patterns in the stance leg during walking. Gait Posture, doi:10.1016/j.gaitpost.2006.06.007.

Cham R, and Redfern MS. (2002). Changes in gait when anticipating slippery floors. Gait Posture, 15:159-171.

Frank JS, Horak FB, and Nutt J. (2000). Centrally initiated postural adjustments in Parkinsonian patients on and off levodopa. J Neurophysiol, 84: 2440-2448.

Heiden TL, Sanderson DJ, Inglis JT, and Siegmund GP. (2006) Adaptations to normal human gait on potentially slippery surfaces: The effects of awareness and prior slip experience. Gait Posture, 24: 237-246.

Hof AL, Gasendam MGJ, and Sinke WE. (2005). The condition for dynamic stability. J Biomechanics, 38: 1-8.

Horak FB, Diener HC, and Nashner LM. (1989). Influence of central set on human postural responses. $J$ Neurophysiol, 61: 841-853.

Horak FB, Frank J, and Nutt J. (1996). Effects of dopamine on postural control in Parkinsonian subjects: Scaling, set, and tone. J Neurophysiol, 75(6): 2380-2396.

Lockhart TE, Smith JL, and Woldstad JC. (2005). Effects of aging on the biomechanics of slips and falls. Human Factors, 47(4): 708-729.

Lockhart TE, Spaulding JM, and Park SH. (2006). Age-related slip avoidance strategy while walking over a known slippery floor surface. Gait Posture, doi:10.1016/j.gaitpost.2006.08.009.

Marigold DS, and Patla AE. (2002). Strategies for dynamic stability during locomotion on a slippery surface: Effects of prior experience and knowledge. J Neurophysiol, 88: 339-353.

Marigold DS, Bethune AJ, and Patla AE. (2003). Role of the unperturbed limb and arms in the reactive recovery response to an unexpected slip during locomotion. J Neurophysiol, 89: 1727-1737.

Morris ME. (2006). Locomotor training in people with Parkinson disease. Physical Ther, 86(10): 1426-1435.

Morris ME, Huxham F, McGinley J, Dodd K, and Iansek R. (2001). Review: The biomechanics and motor control of gait in Parkinson disease. Clinical Biomechanics, 16: 459-470. 
Moyer BE, Chambers AJ, Redfern MS, and Cham R. (2006). Gait parameters as predictors of slip severity in younger and older adults. Ergonomics, 49(4): 329-343.

Myung R, and Smith JL. (1997). The effect of load carrying and floor contaminants on slip and fall parameters. Ergonomics, 40(2): 235-246.

Perry SD, McIlroy WE, and Maki BE. (2000). The role of plantar cutaneous mechanoreceptors in the control of compensatory stepping reactions evoked by unpredictable, multi-directional perturbation. Brain Res, 877: 401-406.

Tang PF, and Woollacott MH. (1998). Inefficient postural responses to unexpected slips during walking in older adults. J Gerontol: MED SCI, 53A(6): M471-M480.

Tang PF, and Woollacott MH. (1999). Phase-dependent modulation of proximal and distal postural responses to slips in young and older adults. J Gerontol: MED SCI, 54A(2): M89-M102.

Tirosh O, and Sparrow WA. (2004). Gait termination in young and older adults: Effects of stopping stimulus probability and stimulus delay. Gait Posture, 19: 243-251.

Troy KL, and Grabiner MD. (2006). Recovery responses to surrogate slipping tasks differ from responses to actual slips. Gait Posture, doi:10.1016/j.gaitpost.2005.09.009. 
CHAPTER 6: GENERAL DISCUSSION AND CONCLUSIONS 


\subsection{DISCUSSION}

Investigation into the control of dynamic stability on a slippery surface revealed a generalized slip response. The slippery surface reduces the possible use of friction and, therefore, the manipulation of ground reaction forces in the gait termination program. The COM lowers, arms raise, and the trail limb steps shorter to help stabilize balance during the slip. The muscles of the lower limb increase their activity to support the lowering body weight and prevent collapse. The difference in the forward momentum with and without the arms shows how the arm raise helps to diminish forward momentum and safely complete the stop. The body also increases the momentum of the COM in the M-L plane perhaps as a result of a transfer of forward to sideways momentum to dissipate the forward momentum caused by the slip and also because of the greater amount of friction in the M-L direction.

The slip response may originate from multiple levels within the central nervous system. During the initial part of the slip, spinal reflexes can monitor the position of the limbs and length of the muscles. Early muscular responses ( $\sim 40 \mathrm{~ms}$ after the perturbation) would reflect the ability of the spinal cord to correct for an unexpected change in muscle length (Pearson and Gordon 2000). Later muscular and behavioural responses reflect supraspinal regulation of the motor program to control dynamic stability and effectively complete the gait termination program. The cortex, cerebellum, lateral vestibular nucleus, and reticulo-spinal tract (Pearson and Gordon 2000), for instance, could be monitoring body and limb position to correct for any deviations caused by the slip. Further exposure to the slippery surface, with knowledge of the surface characteristics, develops an adaptive 
movement strategy. This motor learning would involve not only the supraspinal structures monitoring dynamic stability but include those areas of the brain involved in motor learning such as the cerebellum (Ghez and Thach 2000) and basal ganglia (Seger 2006).

Adaptation to stopping on a slippery surface involves proactive strategies to prepare for and minimize the effect of the slippery surface. Stopping on a slippery surface cannot be completely proactive as the slip is not entirely predictable and some reactive response will remain within the movement. The available friction at the shoe-floor interface is decreased in a slip and, therefore, manipulation of the ground reaction forces diminish with repeated exposure as does the angle of the foot on contact with the slippery surface. The flattened foot increases the contact surface area to accommodate for the lack of friction under the foot by increasing the size of the BOS. The COM remains elevated and is moved forward before the slip in order to keep the COM closer to the newly-formed base of support. This elevated and forward strategy allows participants to "surf" on the slippery surface while stopping. The amount of arm movement and muscular activity throughout the slippery stop also decreases suggesting an increased efficiency and ease with the task over repeated trials. All of these adaptations occur quickly within one or two exposures.

Parkinson's disease caused lower walking velocities, shorter steps and instability during gait termination on a non-slippery surface. A widened step may be a compensatory strategy to control for the diminished stability in the plane of progression. Parkinson's disease did not affect the ability to pre-plan gait termination. Gait termination within one step is also possible for someone with PD suggesting that the ability to switch from steady-state 
locomotion to a gait termination program, whether over multiple steps or within one step, is preserved despite a diminished dynamic stability. This ability to stop, however, may become more difficult as the disease progresses, stability continues to decline and festination becomes more prominent (Morris 2006).

Parkinson's disease continued to cause a slower walking velocity and instabilities with repeated exposure to the slippery surface. Parkinson's disease did not affect the ability to learn adaptive gait termination strategies. The presence of external cues available during the trials, however, may have enabled parallel-processing outside of the basal ganglia to facilitate the adaptation to look more like controls (Morris 2006, Morris et al 2001).

The adaptive strategies seen in the PD participants with repeated exposure include a decreased step length and diminished margin of A-P dynamic stability when stepping onto the slippery surface. A decreased step length keeps the COM closer to the boundaries of the dynamic BOS showing feed-forward control of dynamic stability to minimize the perturbations and facilitate a smooth transition between locomotion and gait termination. The BOS increased by means of a longer and wider step to complete the stop. A larger BOS increases stability and the area in which the COM can move and stabilize. The diminished arm raise over subsequent trials shows an increased ease with stopping on a slippery surface. In both the control and PD groups, these adaptations occurred within two or three trials after the unexpected slippery stop, just slower than the young, healthy adults. 


\subsection{CONCLUSIONS}

Gait termination on a slippery surface evokes a generalized slip response involving a lowering of the COM, a shortened subsequent step, an arm raise and an increase in the muscular activity to control dynamic stability. In young, healthy adults, this slip response is diminished over repeated trials as an adaptive movement strategy is developed to stop more efficiently on the slippery surface. Parkinson's disease causes slowness in movement and instability in the dynamic balance during gait termination on a non-slippery surface. Parkinson's disease does not affect the ability to integrate a balance-correcting response into a voluntary gait termination program. The ability to adapt motor patterns in anticipation of a slippery surface was not affected by PD yet the slowness and instability remained. Practice with a slippery surface may help someone with PD develop appropriate balance maintenance strategies. This training should include measures to increase dynamic stability to prevent future falls and fall-related injuries. 


\subsection{REFERENCES}

Bishop MD, Brunt D, Kukulka C, Tillman, MC, and Pathare N. (2003). Braking impulse and muscle activation during unplanned gait termination in human subjects with Parkinsonism. Neurosci Letters, 348: 89-92.

Frank JS, Horak FB, and Nutt J. (2000). Centrally initiated postural adjustments in Parkinsonian patients on and off levodopa. J Neurophysiol, 84: 2440-2448.

Ghez C, and Thach W.T. (2000). The cerebellum. In Principles of Neural Science (4th Edition). Eds. Kandel, E.R., Schwartz, J.H., \& Jessell, T. M: 832-852.

Morris ME. (2006). Locomotor training in people with Parkinson disease. Physical Therapy, 86(10): 14261435.

Morris ME, Huxham F, McGinley J, Dodd K, and Iansek R. (2001). Review: The biomechanics and motor control of gait in Parkinson disease. Clin Biomech, 16: 459-470.

Pearson K, and Gordon J. (2000). Locomotion. In Principles of Neural Science (4th Edition). Eds. Kandel, E.R., Schwartz, J.H., \& Jessell, T. M: 737-755.

Seger, CA. (2006). The basal ganglia in human learning. Neuroscientist, 12(4): 285-290. 


\section{Appendix A}

Permission from the American Physiological Society 


\section{Penny Ripka}

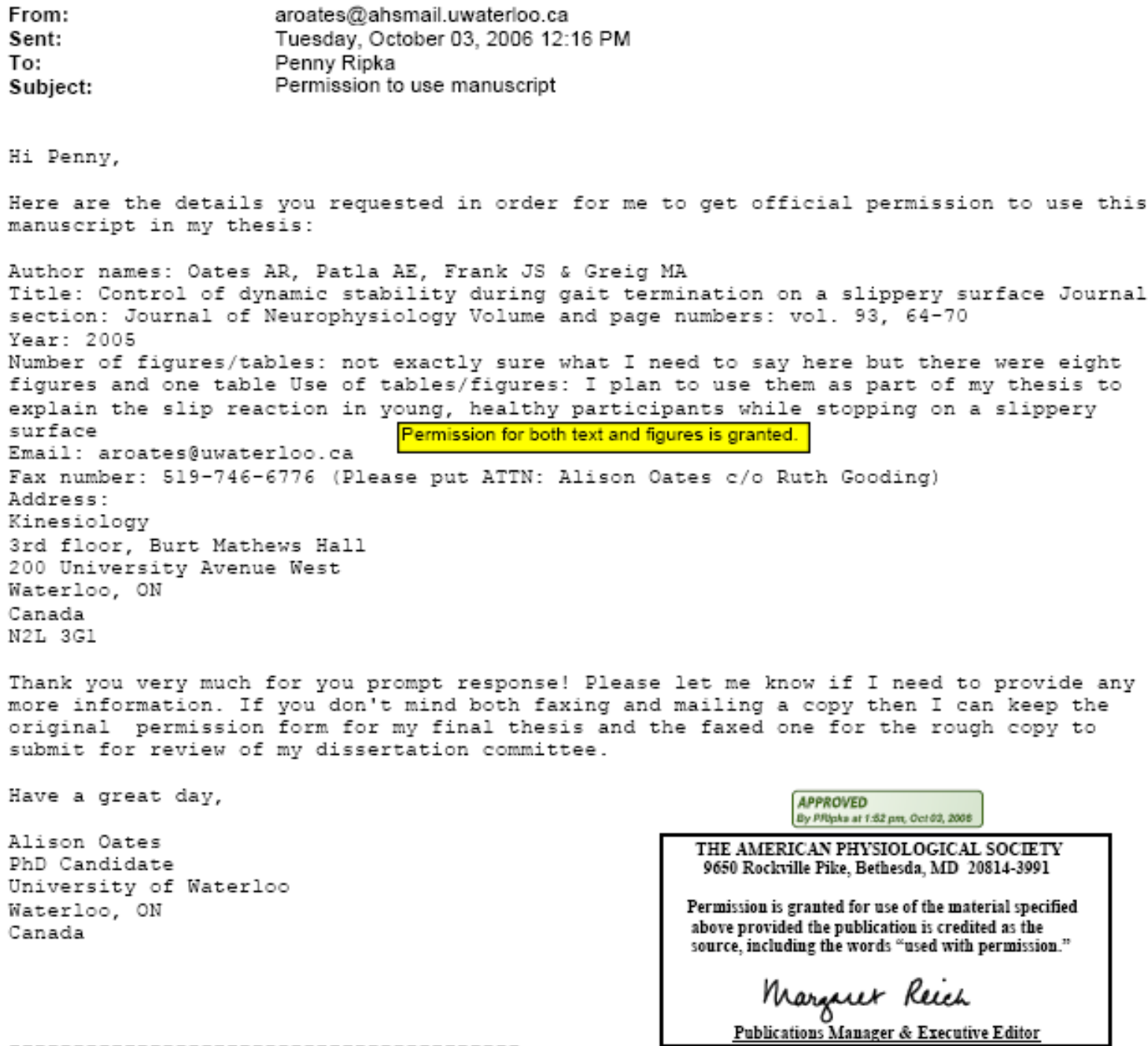

